\title{
Numerical Methods for Mean Field Games and Mean Field Type Control
}

\author{
Mathieu Laurière
}

\begin{abstract}
Mean Field Games (MFG) have been introduced to tackle games with a large number of competing players. Considering the limit when the number of players is infinite, Nash equilibria are studied by considering the interaction of a typical player with the population's distribution. The situation in which the players cooperate corresponds to Mean Field Control (MFC) problems, which can also be viewed as optimal control problems driven by a McKean-Vlasov dynamics. These two types of problems have found a wide range of potential applications, for which numerical methods play a key role since most models do not have analytical solutions. In these notes, we review several aspects of numerical methods for MFG and MFC. We start by presenting some heuristics in a basic linear-quadratic setting. We then discuss numerical schemes for forward-backward systems of partial differential equations (PDEs), optimization techniques for variational problems driven by a Kolmogorov-Fokker-Planck PDE, an approach based on a monotone operator viewpoint, and stochastic methods relying on machine learning tools.
\end{abstract}

\section{Contents}

\section{Introduction}

1.1. Outline

1.2. Definition of the problems and notation 3

2. Warm-up: Linear-Quadratic problems

2.1. Problem definition and MFG solution 5

2.2. Time discretization 6

2.3. Picard (fixed point) iterations 6

2.4. Fictitious play 9

2.5. Newton iterations

2.6. Mean Field Type Control and the Price of Anarchy 10

3. PDE Systems and Numerical Schemes 13

3.1. Optimality condition through PDE systems 13

3.2. A Finite difference scheme

3.3. A Semi-Lagrangian scheme 18

4. Algorithms to solve the discrete schemes 22

4.1. Picard (fixed point) iterations 22

2000 Mathematics Subject Classification. Primary 91-08, 91A13, 93E20, 91A23.

The author was partially supported by NSF DMS-1716673 and ARO W911NF-17-1-0578. 
4.2. Numerical illustration: an example with concentration of mass 22

4.3. Newton method 24

4.4. Numerical illustration: evacuation of a room with congestion effects 25

5. Optimization methods for MFC and variational MFGs 28

5.1. Variational formulation of MFC and MFG 28

5.2. A PDE driven optimal control problem 229

5.3. Discrete version of the PDE driven optimal control problem

5.4. ADMM and Primal-dual method 32

5.5. Numerical illustration: crowd motion without diffusion 34

6. A Method based on monotone operators 37

6.1. A Monotonic flow 37

6.2. Numerical illustration 38

7. Methods based on approximation by neural networks 41

7.1. Direct optimization for MFC 41

7.2. Solving the finite state master equation 45

8. A Glance at model-free methods 52

8.1. Reinforcement learning for mean field control 52

8.2. Reinforcement learning in mean field games 55

9. Conclusion and perspectives

References 58

These lecture notes are to supplement the AMS short course on mean field games on January 13-14, 2020, and are meant to be an introduction to numerical methods for mean field games. See also the other proceedings of the AMS Short Course on Mean Field Games [50, 63, 79, 90, 106].

\section{Introduction}

Mean field games (MFGs for short) have been introduced to study differential games with an infinite number of players, under indistinguishability and symmetry assumptions. This theory has been introduced by J.-M. Lasry and P-L. Lions [91, 92, 93, and independently by Caines, Huang, and Malhamé under the name of Nash Certainty Equivalence principle [87, 86. While MFGs correspond to noncooperative games and focus on Nash equilibria, the cooperative counterpart has been studied under the name mean field type control (or mean field control, or MFC for short) or optimal control of McKean-Vlasov dynamics [51, 30]. For more details, we refer to Lions' lectures at Collège de France [96 and the notes by Cardaliaguet [41, as well as the books by Bensoussan, Frehse and Yam [30, Gomes, Pimentel and Voskanyan [75, Carmona and Delarue [52, 53, and the surveys by Gomes and Saúde [76] and Caines, Huang and Malhamé [38]. Mean field games and mean field control problems have attracted a surge of interest and have found numerous applications, from economics to the study of crowd motion or epidemic models. For concrete applications, it is often important to obtain reliable quantitative results on the solution. Since very few mean field problems have explicit or semi-explicit solutions, the introduction of numerical methods and their analysis are crucial steps in the development of this field. 
The goal of these notes is to provide an introduction to numerical aspects of MFGs and MFC problems. We discuss both numerical schemes and associated algorithms, and illustrate them with numerical examples. In order to alleviate the presentation while conveying the main ideas, the mathematical formalism is not carried out in detail. The interested reader is referred to the references provided in the text for a more rigorous treatment (see in particular the lecture notes [1, 10]).

1.1. Outline. The rest of these notes is organized as follows. In the remainder of this section, we introduce a general framework for the type of problems we will consider. Linear-quadratic problems are used in Section 2 as a testbed to introduce several numerical strategies that can be applied to more general problems, such as fixed point iterations, fictitious play iterations and Newton iterations. Beyond the linear-quadratic structure, optimality conditions in terms of PDE systems are discussed in Section 3 and two numerical schemes to discretize these PDEs are presented: a finite-difference scheme and a semi-Lagrangian scheme. In Section 4 we present some strategies to solve the discrete schemes and provide numerical illustrations, including to crowd motion with congestion effects. Section 5 focuses on MFC problems and MFGs with a variational structure. For such problems, optimization techniques can be used and we present two of them: the Alternating Direction Method of Multipliers and a primal-dual algorithm. For MFGs satisfying a monotonicity condition, a different technique, based on a monotonic flow, is discussed in Section 6. The last two sections present techniques which borrow tools from machine learning. Section 7 presents methods relying on neural network approximation to solve stochastic optimal control problems or PDEs. We apply these methods to MFC and to the (finite state MFG) master equation respectively. Section 8 discusses model-free methods, i.e., methods which learn the solution by trial and error, without knowing the full model. We conclude in Section 9 by mentioning other methods and research directions.

1.2. Definition of the problems and notation. Let $T$ be a fixed time horizon, let $d$ and $k$ be integers and let $\mathcal{Q} \subseteq \mathbb{R}^{d}$ be a spatial domain, which will typically be either the whole space $\mathbb{R}^{d}$ or the unit torus $\mathbb{T}^{d}$. We will use the notation $\mathcal{Q}_{T}=[0, T] \times \mathcal{Q}$, and $\langle\cdot, \cdot\rangle$ for the inner product of two vectors of compatible sizes.

Let $f: \mathcal{Q} \times L^{2}(\mathcal{Q}) \times \mathbb{R}^{k} \rightarrow \mathbb{R},(x, m, \alpha) \mapsto f(x, m, \alpha)$ and $g: \mathcal{Q} \times L^{2}(\mathcal{Q}) \rightarrow$ $\mathbb{R},(x, m) \mapsto g(x, m)$ be respectively a running cost and a terminal cost. Let $\sigma \geq 0$ be a constant parameter for the volatility of the state's evolution. Let $b: \mathcal{Q} \times$ $L^{2}(\mathcal{Q}) \times \mathbb{R}^{k} \rightarrow \mathbb{R}^{d},(x, m, \alpha) \mapsto b(x, m, \alpha)$ be a drift function. These functions could be allowed to also depend on time at the expense of heavier notation. Here, $x, m$ and $\alpha$ play respectively the role of the state of the agent, the mean-field term, (i.e., the population's distribution), and the control used by the agent. In general, the mean-field term is a probability measure, but here, for simplicity, we will assume that this probability measure has a density which is in $L^{2}(\mathcal{Q})$.

We consider the following mean field game. A (mean field) Nash equilibrium consists in a flow of probability densities $\hat{m}: \mathcal{Q}_{T} \rightarrow \mathbb{R}$ and a feedback control $\hat{\alpha}: \mathcal{Q}_{T} \rightarrow \mathbb{R}^{k}$ satisfying the following two conditions:

(1) $\hat{\alpha}$ minimizes $J_{\hat{m}}^{M F G}$ where, for $m \in L^{2}\left(\mathcal{Q}_{T}\right)$,

$$
J_{m}^{M F G}: \alpha \mapsto \mathbb{E}\left[\int_{0}^{T} f\left(X_{t}^{m, \alpha}, m(t, \cdot), \alpha\left(t, X_{t}^{m, \alpha}\right)\right) d t+g\left(X_{T}^{m, \alpha}, m(T, \cdot)\right)\right]
$$


under the constraint that the process $X^{m, \alpha}=\left(X_{t}^{m, \alpha}\right)_{t \geq 0}$ solves the stochastic differential equation (SDE)

$$
d X_{t}^{m, \alpha}=b\left(X_{t}^{m, \alpha}, m(t, \cdot), \alpha\left(t, X_{t}^{m, \alpha}\right)\right) d t+\sigma d W_{t}, \quad t \geq 0,
$$

where $W$ is a standard $d$-dimensional Brownian motion, and $X_{0}^{m, \alpha}$ has distribution with density $m_{0}$ given;

(2) For all $t \in[0, T], \hat{m}(t, \cdot)$ is the probability density of the law of $X_{t}^{\hat{m}, \hat{\alpha}}$.

In the definition (1.1) of the cost function, the subscript $m$ is used to emphasize the dependence on the mean-field flow, which is fixed when an infinitesimal agent performs their optimization. The second condition ensures that if all the players use the control $\hat{\alpha}$ computed in the first point, the law of their individual states is indeed $\hat{m}$.

Using the same running cost, terminal costs, drift function and volatility, we can look at the corresponding mean field control (MFC for short) problem. This problem corresponds to a social optimum and is phrased as an optimal control problem. It can be interpreted as a situation in which all the agents cooperate to minimize the average cost. The goal is to find a feedback control $\alpha^{*}: \mathcal{Q}_{T} \rightarrow \mathbb{R}^{k}$ minimizing

$$
J^{M F C}: \alpha \mapsto \mathbb{E}\left[\int_{0}^{T} f\left(X_{t}^{\alpha}, m^{\alpha}(t, \cdot), \alpha\left(t, X_{t}^{\alpha}\right)\right) d t+g\left(X_{T}^{\alpha}, m^{\alpha}(T, \cdot)\right)\right]
$$

where $m^{\alpha}(\cdot, t)$ is the probability density of the law of $X_{t}^{\alpha}$, under the constraint that the process $X^{\alpha}=\left(X_{t}^{\alpha}\right)_{t \geq 0}$ solves the SDE

$$
d X_{t}^{\alpha}=b\left(X_{t}^{\alpha}, m^{\alpha}(t, \cdot), \alpha\left(t, X_{t}^{\alpha}\right)\right) d t+\sigma d W_{t}, \quad t \geq 0,
$$

and $X_{0}^{\alpha}$ has distribution with density $m_{0}$ given.

Besides the interpretation as a social optimum for a large population of cooperative players, MFC problems also arise in risk management 19 or in optimal control with a cost involving a conditional expectation 101, 11.

Let $m^{*}=m^{\alpha^{*}}$ denote the optimal mean-field flow. Then, we have, for any MFG equilibrium $(\hat{\alpha}, \hat{m})$ :

$$
J^{M F C}\left(\alpha^{*}\right)=J_{m^{*}}^{M F G}\left(\alpha^{*}\right) \leq J_{\hat{m}}^{M F G}(\hat{\alpha}) .
$$

In general the inequality is strict, which leads to the notion of price of anarchy as we will illustrate below.

While we will mostly focus on the problems as introduced above, the ergodic setting is discussed in Section 6 and finite state MFGs are considered in $\S 7.2$. 


\section{Warm-up: Linear-Quadratic problems}

We start by considering the important subclass of problems in which, on the one hand, the dynamics is linear in the state, the control and the mean of the state (and possibly the mean of the control), and, on the other hand, the cost is quadratic in these variables. Then, the MFG and MFC optimal controls $\hat{\alpha}$ and $\alpha^{*}$ can be characterized by a system of backward ODEs, which is in general coupled with a forward ODE for the evolution of the mean of the state 1

2.1. Problem definition and MFG solution. We borrow the following example from [30, Chapter 6]. For ease of presentation, we restrict our attention to the one-dimensional case, i.e., $d=k=1$. Consider

$$
\begin{aligned}
f(x, m, \alpha) & =\frac{1}{2}\left[Q x^{2}+\bar{Q}\left(x-S \int_{\mathcal{Q}} \xi m(\xi) d \xi\right)^{2}+C \alpha^{2}\right] \\
g(x, m) & =\frac{1}{2}\left[Q_{T} x^{2}+\bar{Q}_{T}\left(x-S_{T} \int_{\mathcal{Q}} \xi m(\xi) d \xi\right)^{2}\right] \\
b(x, m, \alpha) & =A x+\bar{A} \int_{\mathcal{Q}} \xi m(\xi) d \xi+B \alpha,
\end{aligned}
$$

where $Q, C, \bar{Q}, Q_{T}, \bar{Q}_{T}$ are non-negative constants, and $A, \bar{A}, S, S_{T}$ and $B$ are constants. Let $\nu=\frac{1}{2} \sigma^{2}$. We consider that the initial distribution is a normal $\mathcal{N}\left(\bar{x}_{0}, \sigma_{0}^{2}\right)$ for some $\bar{x}_{0} \in \mathbb{R}$ and $\sigma_{0}>0$.

Under suitable conditions on these coefficients, the MFG for the above model has a unique solution $(\hat{\alpha}, \hat{m})$ which satisfies the following. The proof relies on dynamic programming and on a suitable ansatz for the value function. See e.g. 30 , Chapter 6] for more details. The solution is given by:

$$
\left\{\begin{aligned}
\int_{\mathcal{Q}} \xi \hat{m}(t, \xi) d \xi & =z_{t} \\
\hat{\alpha}(t, x) & =-B\left(p_{t} x+r_{t}\right) / C \\
J_{\hat{m}}^{M F G}(\hat{\alpha}) & =\int_{\mathcal{Q}} u(0, \xi) m_{0}(\xi) d \xi=\frac{1}{2} p_{0}\left(\sigma_{0}^{2}+\bar{x}_{0}^{2}\right)+r_{0} \bar{x}_{0}+s_{0}, \\
u(t, x) & =\frac{1}{2} p_{t} x^{2}+r_{t} x+s_{t},
\end{aligned}\right.
$$

where $(z, p, r, s)$ solve the following system of ordinary differential equations (ODEs):

$$
\left\{\begin{aligned}
\frac{d z}{d t} & =\left(A+\bar{A}-B^{2} C^{-1} p_{t}\right) z_{t}-B^{2} C^{-1} r_{t}, & z_{0} & =\int_{\mathcal{Q}} \xi m_{0}(\xi) d \xi \\
-\frac{d p}{d t} & =2 A p_{t}-B^{2} C^{-1} p_{t}^{2}+Q+\bar{Q}, & p_{T} & =Q_{T}+\bar{Q}_{T} \\
-\frac{d r}{d t} & =\left(A-B^{2} C^{-1} p_{t}\right) r_{t}+\left(p_{t} \bar{A}-\bar{Q} S\right) z_{t}, & r_{T} & =-\bar{Q}_{T} S_{T} z_{T} \\
-\frac{d s}{d t} & =\nu p_{t}-\frac{1}{2} B^{2} C^{-1} r_{t}^{2}+r_{t} \bar{A} z_{t}+\frac{1}{2} S^{2} \bar{Q} z_{t}^{2}, & s_{T} & =\frac{1}{2} \bar{Q}_{T} S_{T}^{2} z_{T}^{2}
\end{aligned}\right.
$$

${ }^{1}$ In some cases, a suitable parametrization leads to a decoupled ODE system; see e.g. $7 \mathbf{7 9}$ for an example. 
In this system, $z$ represents the mean of the population's distribution whereas $r$ (together with $p$ ) characterizes the best response. In fact, $u(t, x)=\frac{1}{2} p_{t} x^{2}+r_{t} x+s_{t}$ is the value function of an infinitesimal player when the population is in the Nash equilibrium. The last equation admits an explicit solution, in terms of $z, p$ and $r$. The second equation can be solved independently of the other ones. It is a Riccati equation and, under suitable conditions, admits a unique positive (symmetric if $d>$ 1) solution. The first and the third equations are coupled. Note that the equation for $z$ is forward in time whereas the equation for $r$ is backward in time. This forward-backward structure prevents the use of a simple time-marching method to solve numerically the system. This obstacle is at the heart of numerical methods for MFG and MFC problems. Using this LQ example to provide the main ideas, we present below a few strategies to tackle forward-backward systems. In the rest of this section, we assume that $p$ solving $2.2 \mathrm{~b}$ is given and we focus on the system 2.2a)-2.2c).

2.2. Time discretization. In order to solve this ODE system numerically, we first partition the interval $[0, T]$ into $N_{T}$ subintervals, where $N_{T}$ is a positive integer. Let $\Delta t=1 / N_{T}$ and $t_{i}=i \times \Delta t$ for $i=0, \ldots, N_{T}$. The functions of time $\left(z_{t}\right)_{t \in[0, T]}$, $\left(p_{t}\right)_{t \in[0, T]},\left(r_{t}\right)_{t \in[0, T]}$ and $\left(s_{t}\right)_{t \in[0, T]}$ are approximated respectively by vectors $Z=$ $\left(Z^{n}\right)_{n=0, \ldots, N_{T}}, P=\left(P^{n}\right)_{n=0, \ldots, N_{T}}, R=\left(R^{n}\right)_{n=0, \ldots, N_{T}}$ and $S=\left(S^{n}\right)_{n=0, \ldots, N_{T}}$. The ODE system $2.2 \mathrm{a}-(2.2 \mathrm{c})$ is then replaced by a finite-difference system. Focusing on 2.2a) and (2.2c), we consider the following system: for $n \in\left\{0, \ldots, N_{T}-1\right\}$,

$$
\left\{\begin{array}{l}
\frac{Z^{n+1}-Z^{n}}{\Delta t}=\left(A+\bar{A}-B^{2} C^{-1} P^{n}\right) Z^{n+1}-B^{2} C^{-1} R^{n}, \\
Z^{0}=\bar{x}_{0}, \\
-\frac{R^{n+1}-R^{n}}{\Delta t}=\left(A-B^{2} C^{-1} P^{n}\right) R^{n}+\left(P^{n} \bar{A}-\bar{Q} S\right) Z^{n+1}, \\
R^{N_{T}}=-\bar{Q}_{T} S_{T} Z^{N_{T}} .
\end{array}\right.
$$

Note that for each equation (considered separately) the scheme is semi-implicit, since the first one is forward in time and the second in backward in time. This finite-difference system can be rewritten in a matrix form:

$$
\mathbf{M}\left(\begin{array}{l}
Z \\
R
\end{array}\right)+\mathbf{B}=0
$$

where $\mathbf{M} \in \mathbb{R}^{\left(N_{T}+1\right) \times\left(N_{T}+1\right)}$ and $\mathbf{B} \in \mathbb{R}^{\left(N_{T}+1\right)}$ are defined in order to take into account the dynamics as well as the initial and terminal conditions. One can thus obtain $(Z, R)$ directly by solving this linear system. However, this is specific to the LQ setting. As we will see in the next section, forward-backward PDE systems appearing in MFG are generally not linear. Hence we present in the rest of this section several solution strategies which do not exploit the linear structure and will be useful in a more general setting. The LQ problem is simply used as an illustration to provide the main ideas behind these methods.

2.3. Picard (fixed point) iterations. To alleviate the presentation, we keep the ODE notation, i.e., 2.2a) \& 2.2c), although for the implementation, we use their finite-difference counterpart, i.e., (2.3a) \& 2.3b). A first approach consists in solving alternatively each ODE. Starting from an initial guess $z^{(0)}$, solve $(2.2 \mathrm{c})$ in which $z$ is replaced by $z^{(0)}$. Denoting $r^{(1)}$ the solution, solve 2.2a in which $r$ is replaced 
by $r^{(1)}$. Then repeat these steps, plugging $z^{(1)}$ in $2.2 \mathrm{c}$ and so on. This procedure defines a map $\varphi$ such that $\varphi\left(z^{(0)}\right)=z^{(1)}$. If this map is a strict contraction, then the above iterations converge. A pseudo-code is given in Algorithm 1 where (plain) Picard iterations correspond to the case $\delta(\cdot) \equiv 0$, and an example is displayed in Figure 1. Here we used the values of parameters described in Table 1 for Test case 1. Instead of fixing a priori a number of iterations, we can also consider a stopping criterion of the form:

$$
\left\|r^{(\mathrm{k}+1)}-r^{(\mathrm{k})}\right\|<\varepsilon, \quad \text { and } \quad\left\|z^{(\mathrm{k}+1)}-z^{(\mathrm{k})}\right\|<\varepsilon,
$$

for some threshold $\varepsilon>0$.

\begin{tabular}{c|c|c|c|c|c|c}
\hline Parameters & $Q, R, S, S_{T}, A, B, \sigma, \bar{x}_{0}$ & $\bar{Q}$ & $\bar{Q}_{T}$ & $Q_{T}$ & $\bar{A}$ & $\sigma_{0}$ \\
\hline Test case 1 & 1 & 1 & 1 & 1 & 1 & 0.2 \\
\hline Test case 2 & 1 & 1 & 2.45 & 1 & 1 & 0.2 \\
\hline Test case 3 & 1 & 0 & 0 & 1 & {$[0,20]$} & 0.2 \\
\hline Test case 4 & 1 & 0 & {$[0,20]$} & 1 & 1 & 0.2 \\
\hline Test case 5 & 1 & 0 & 1 & {$[0,20]$} & 1 & 0.2 \\
\hline
\end{tabular}

TABLE 1. Parameters values for the LQ model introduced in $\S 2.1$. The test cases are discussed in the text.
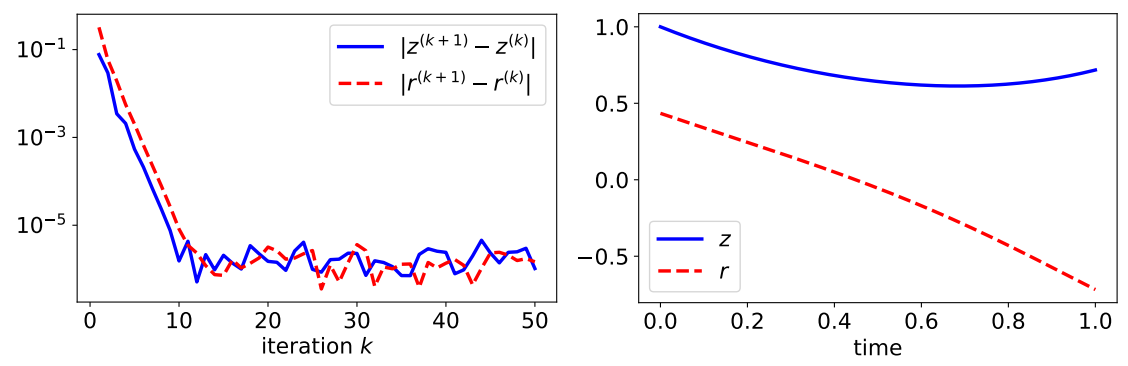

Figure 1. Picard iterations without damping for Test case 1 (see Table 1): $L^{2}$ difference between two successive iterates (left) and $z^{(50)}$ and $r^{(50)}$ (right). The curves related to $z$ and $r$ are respectively in blue (full line) and in red (dashed line).

However, this procedure fails on many examples. Figure 2 based on Test case 2 in Table 1 provides an illustration: here we see that $z^{(\mathrm{k})}$ and $r^{(\mathrm{k})}$ end up reacting to each other from an iteration to the next one, and the overall process diverge. In the framework of MFG, a possible interpretation for this phenomenon is the following (recall that $z$ corresponds to the equilibrium mean and $r$ is part of the equilibrium control). Given a trajectory for the mean-field term, each agent compute their best response to this crowd behavior. Applying this best response leads to a new trajectory for the mean-field term. Computing once again the best response leads back to the first trajectory. This type of phenomenon is also well-known in twoplayer games. 

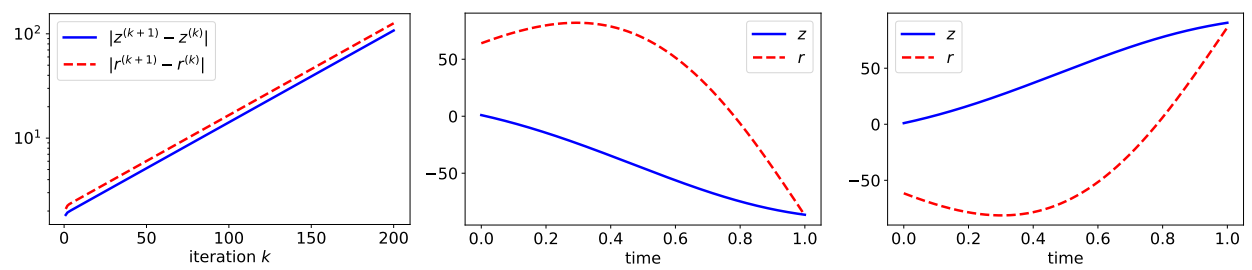

Figure 2. Picard iterations without damping for Test case 2 (see Table 1): $L^{2}$ difference between two successive iterates (left), $z^{(199)}$ and $r^{(199)}$ (middle) and $z^{(200)}$ and $r^{(200)}$ (right). The curves related to $z$ and $r$ are respectively in blue (full line) and in red (dashed line).

A simple approach to try and fix the above method is to add damping to the iterations. Let $\omega \in[0,1)$ be a damping parameter. At iteration $\mathrm{k} \geq 0$, letting $z^{(\mathrm{k}+1)}$ be the solution to (2.2a) with $r^{(\mathrm{k})}$, instead of directly plugging this new mean-field trajectory in $2.2 \mathrm{c}$, we define

$$
\tilde{z}^{(\mathrm{k}+1)}=\omega \tilde{z}^{(\mathrm{k})}+(1-\omega) z^{(\mathrm{k}+1)}
$$

and plug this trajectory in $2.2 \mathrm{c}$ to compute $r^{(\mathrm{k}+1)}$. This corresponds to taking $\delta(\cdot) \equiv \omega$ in Algorithm 1. Such a modification of the plain Picard iterations typically helps to ensure convergence of the iterations but the choice of the damping parameter is not obvious: if it is too small, convergence may still fail, whereas if it is too large, convergence will be very slow. See Figure 3 and Figure 4
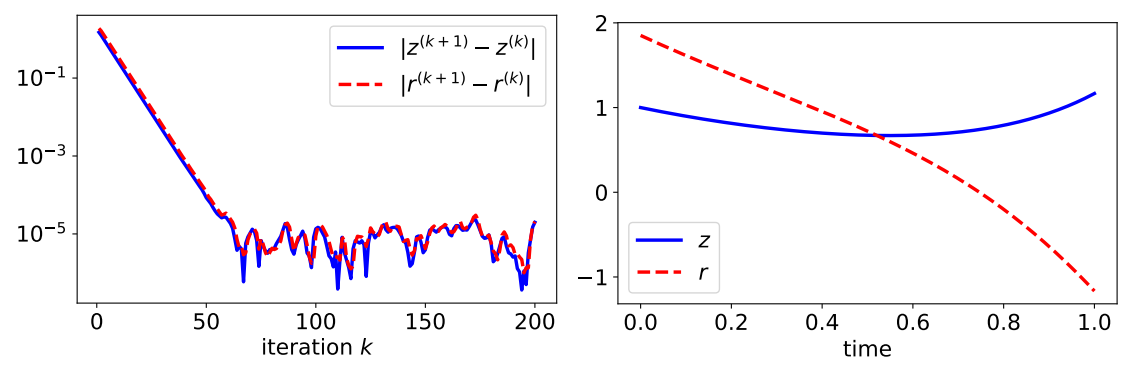

Figure 3. Picard iterations with constant damping $\omega=0.1$ for Test case 2 (see Table 1): $L^{2}$ difference between two successive iterates (left) and $z^{(200)}$ and $r^{(200)}$ (right). The curves related to $z$ and $r$ are respectively in blue (full line) and in red (dashed line). 

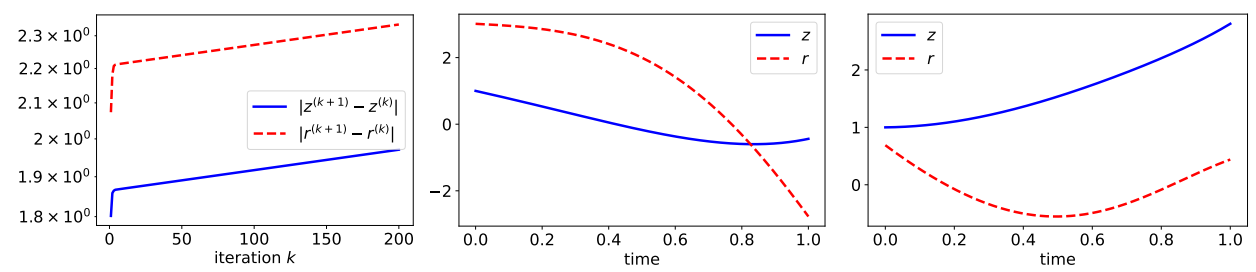

Figure 4. Picard iterations with constant damping $\omega=0.01$ for Test case 2 (see Table 1): $L^{2}$ difference between two successive iterates (left), $z^{(199)}$ and $r^{(199)}$ (middle) and $z^{(200)}$ and $r^{(200)}$ (right). The curves related to $z$ and $r$ are respectively in blue (full line) and in red (dashed line).

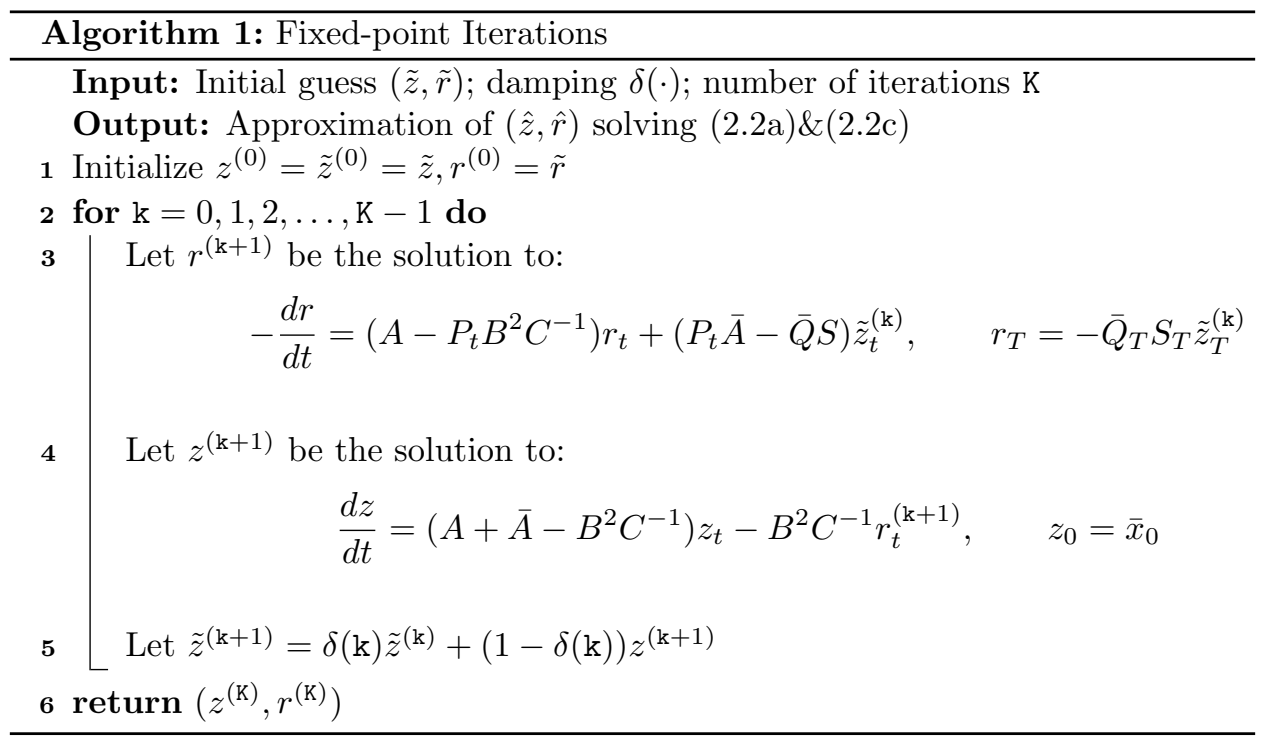

2.4. Fictitious play. Another procedure, which has been widely studied in algorithmic game theory, is the so-called fictitious play. It has also drawn interest in the MFG community [45, 84, 68, 103. Once again, it amounts to update in turn the mean-field term and the control, but here the control is computed as the best response to a weighted average of the mean-field terms in previous iterations. In the context of the LQ example studied here, one can recast this procedure as follows: $r^{(\mathrm{k}+1)}$ is computed given $\tilde{z}^{(\mathrm{k})}$ and

$$
\tilde{z}^{(\mathrm{k}+1)}=\frac{\mathrm{k}}{\mathrm{k}+1} \tilde{z}^{(\mathrm{k})}+\frac{1}{\mathrm{k}+1} z^{(\mathrm{k}+1)}
$$

where $z^{(\mathrm{k}+1)}$ solves $2.2 \mathrm{a}$ with $r$ replaced by $r^{(\mathrm{k}+1)}$. This corresponds to Algorithm 1 with $\delta(\mathrm{k})=\frac{\mathrm{k}}{\mathrm{k}+1}$.

An important advantage of this method is that it can be proved to converge under less stringent conditions than Picard iterations, see 45. However, the damping effect becomes stronger as the number of iterations increases, which means that convergence can be slower. This is illustrated in Figure 5. Heuristics to improve the convergence have been proposed in $\mathbf{8 4}$. 

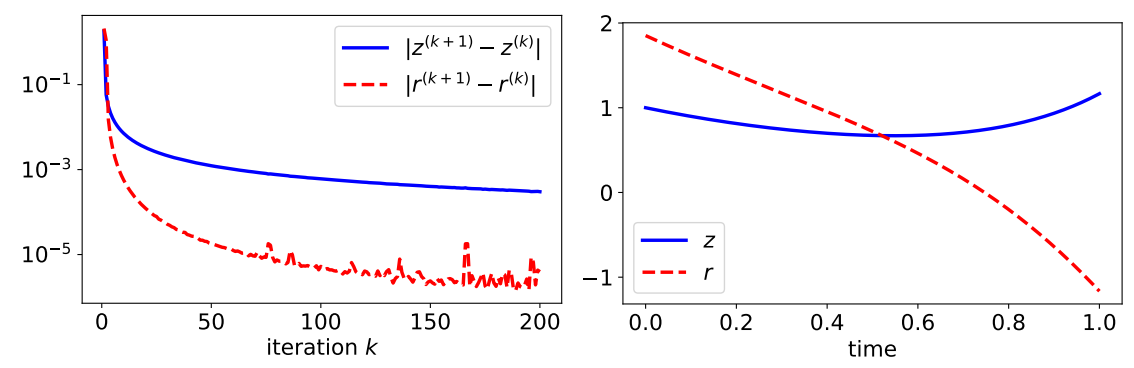

Figure 5. Fictitious play iterations for Test case 2 (see Table 1): $L^{2}$ difference between two successive iterates (left) and $z^{(200)}$ and $r^{(200)}$ (right). The curves related to $z$ and $r$ are respectively in blue (full line) and in red (dashed line).

2.5. Newton iterations. Instead of updating alternatively $z$ and $r$ by solving a forward equation and then a backward equation, another approach consists in solving the whole forward-backward system using Newton iterations. After discretizing time as in $\S 2.2$, the idea is to view the system $(2.3 \mathrm{a}) \&(2.3 \mathrm{~b})$ as the zero of an operator $\mathcal{F}: \mathbb{R}^{2\left(N_{T}+1\right)} \rightarrow \mathbb{R}^{2\left(N_{T}+1\right)}$ defined as:

$$
(Z, R) \text { solve 2.3a }-2.3 \mathrm{~b} \Leftrightarrow \mathcal{F}(Z, R)=0 .
$$

Note that $\mathcal{F}$ must take into account the initial and terminal conditions. Denoting by $D \mathcal{F}$ the differential of this operator, Algorithm 2 summarizes Newton iterations to find a zero of $\mathcal{F}$. In the present LQ example, this operator is linear hence solving 2.5 is just as straightforward as solving directly the linear system 2.4 of interest. See Fig 6. However, this strategy based on Newton iterations will be particularly useful when solving forward-backward systems of PDEs which are not linear (see $\S 4.3$ ).

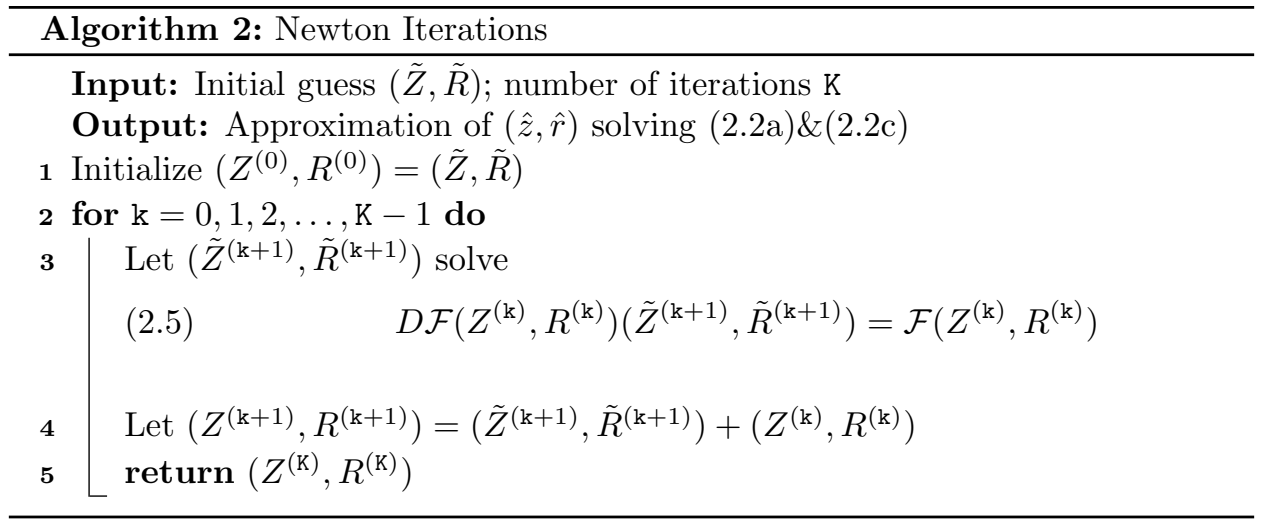

2.6. Mean Field Type Control and the Price of Anarchy. Using the linear dynamics and quadratic costs introduced $\S 2.1$. we now turn our attention to the corresponding MFC problem. For this problem too, the solution can be characterized using a system of ODEs. More precisely, denoting by $\alpha^{*}$ and $m^{*}$ 

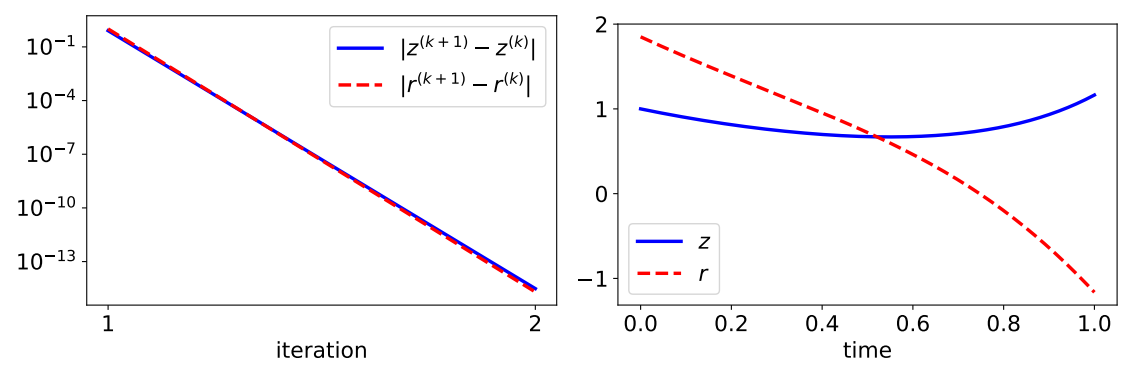

Figure 6 . Newton iterations for Test case 2 (see Table 1): $L^{2}$ difference between two successive iterates (left) and $z^{(200)}$ and $r^{(200)}$ (right). The curves related to $z$ and $r$ are respectively in blue (full line) and in red (dashed line).

respectively the optimal control and the induced flow of densities, we have

$$
\left\{\begin{aligned}
\int \xi m^{*}(t, \xi) d \xi & =\check{z}_{t} \\
\alpha^{*}(t, x) & =-B\left(\check{p}_{t} x+\check{r}_{t}\right) / C \\
J^{M F C}\left(\alpha^{*}\right)= & \frac{1}{2} \check{p}_{0}\left(\sigma_{0}^{2}+\bar{x}_{0}^{2}\right)+\check{r}_{0} \bar{x}_{0}+\check{s}_{0}+\left(1-S_{T}\right) \bar{Q}_{T} S_{T} \check{z}_{T}^{2} \\
& -\int_{0}^{T}\left[\left(\check{p}_{t} \check{z}_{t}+\check{r}_{t}\right) \bar{A} \check{z}_{t}-\left(1-S_{t}\right) \bar{Q} S \check{z}_{t}^{2}\right] d t
\end{aligned}\right.
$$

where $(\check{z}, \check{p}, \check{r}, \check{s})$ solve the following system of ordinary differential equations (ODEs):

$$
\left\{\begin{aligned}
\frac{d \check{z}}{d t} & =\left(A+\bar{A}-B^{2} C^{-1}\right) \check{z}_{t}-B^{2} C^{-1} \check{r}_{t}, & & \check{z}_{0}=\bar{x}_{0}, \\
-\frac{d \check{p}}{d t} & =2 A \check{p}_{t}-B^{2} C^{-1} \check{p}_{t}^{2}+Q+\bar{Q}, & & \check{p}_{T}=Q_{T}+\bar{Q}_{T}, \\
-\frac{d \check{r}}{d t} & =\left(A+\bar{A}-\check{p}_{t} B^{2} C^{-1}\right) \check{r}_{t}+\left(2 \check{p}_{t} \bar{A}-2 \bar{Q} S+\bar{Q} S^{2}\right) \check{z}_{t}, & \check{r}_{T} & =-\bar{Q}_{T} S_{T} \check{z}_{T} \\
-\frac{d s}{d t} & =\nu \check{p}_{t}-\frac{1}{2} B^{2} C^{-1} \check{r}_{t}^{2}+\check{r}_{t} \bar{A} \check{z}_{t}+\frac{1}{2} S^{2} \bar{Q} \check{z}_{t}^{2}, & & \check{s}_{T}=\frac{1}{2} \bar{Q}_{T} S_{T}^{2} \check{z}_{T}^{2}
\end{aligned}\right.
$$

Note that this system is very similar to 2.2a $-2.2 \mathrm{~d}$ ) except that the ODEs for $r$ and $\check{r}$ are different. Furthermore, the expressions for $J^{M F G}$ and $J^{M F C}$ are also different. This is due to the fact that, in the MFC, the function $\check{u}(t, x)=\frac{1}{2} \check{p}_{t} x^{2}+\check{r}_{t} x+\check{s}_{t}$ is not the value function of the control problem but rather an adjoint state, as we shall see in Section 3

Since MFC corresponds to a social optimum, the price paid by a typical player can only be lower than in a MFG (with the same costs and dynamics). In other words, the price of anarchy (PoA), defined as:

$$
\operatorname{PoA}=\frac{J_{\hat{m}}^{M F G}(\hat{\alpha})}{J^{M F C}\left(\alpha^{*}\right)}
$$

is at least 1. Figure 7 illustrates the price of anarchy when $\bar{A}, \bar{Q}$ or $Q_{T}$ vary. In particular, in Test case $3, \bar{Q}=\bar{Q}_{T}=0$ and when $\bar{A}=0$ too, the price of anarchy is 1 , see Table 1. Indeed, in this scenario, the mean-field interactions completely 
disappear from the cost and the dynamics, hence MFG and MFC amount to the same problem. Test cases 4 and 5 illustrate the dependence of the price of anarchy with respect to $\bar{Q}_{T}$ and $Q_{T}$ respectively. For more details on the price of anarchy in LQ MFG and MFC, see [54].
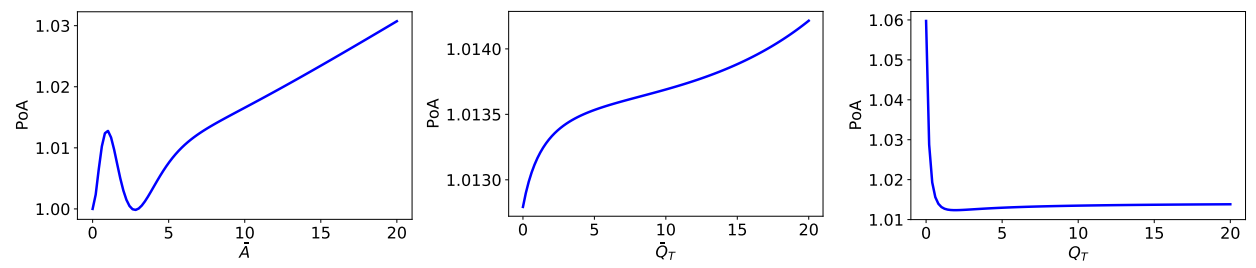

Figure 7. Price of anarchy for Test case 3 (left), Test case 4 (middle) and Test case 5 (right). See Table 1 for the parameters values. 


\section{PDE Systems and Numerical Schemes}

In general, besides the LQ case, optimality conditions for MFG and MFC can not be phrased in terms of ODEs. The two main approaches are through systems of partial differential equations (PDEs) or systems stochastic differential equations (SDEs). In both cases, these systems have a forward-backward structure. A third approach is via the so-called master equation, which we will discuss in $\S 7.2$. For now, we focus on the first approach, which leads to a system composed of a forward Kolmogorov-Fokker-Planck PDE for the distribution of the population's state coupled with a backward Hamilton-Jacobi-Bellman equation for the value function of a typical player. To solve numerically this PDE system, the first question that arises is the approximation of functions of space and time. A possible approach is to consider a space-time mesh and approximate these functions by their values at the points of the mesh. The functions are approximated by vectors, which satisfy finite difference equations coming from a suitable numerical scheme of the PDEs.

3.1. Optimality condition through PDE systems. For the sake of simplicity, unless otherwise specified, we consider the $d$-dimensional torus for the spatial domain, i.e., $\mathcal{Q}=\mathbb{T}^{d}$. We first consider the MFG and then the MFC. Despite their similarities, the two PDE systems have important differences reflecting the differences between the two problems.

Mean field game. We first note that for a given flow of densities $\hat{m}$ and a given feedback control $\alpha$, the density $m_{t}^{\hat{m}, \alpha}$ of the law of $X_{t}^{\hat{m}, \alpha}$ solving (1.2) satisfies the Kolmogorov-Fokker-Planck (KFP) equation:

$\frac{\partial m^{\hat{m}, \alpha}}{\partial t}(t, x)-\nu \Delta m^{\hat{m}, \alpha}(t, x)+\operatorname{div}\left(m^{\hat{m}, \alpha}(t, \cdot) b(\cdot, \hat{m}(t), \alpha(t, \cdot))\right)(x)=0$, in $(0, T] \times \mathbb{T}^{d}$, with the initial condition:

$$
m^{\hat{m}, \alpha}(0, x)=m_{0}(x), \text { in } \mathbb{T}^{d} .
$$

Let $H: \mathbb{T}^{d} \times L^{2}\left(\mathbb{T}^{d}\right) \times \mathbb{R}^{d} \rightarrow \in \mathbb{R}$ be the Hamiltonian of the control problem faced by an infinitesimal agent in the first point above, which is defined by:

$$
H:(x, m, p) \mapsto H(x, m, p)=\max _{\alpha \in \mathbb{R}^{k}}\{-L(x, m, \alpha, p)\},
$$

where $L: \mathbb{T}^{d} \times L^{2}\left(\mathbb{T}^{d}\right) \times \mathbb{R}^{k} \times \mathbb{R}^{d} \rightarrow \mathbb{R}$ is the Lagrangian, defined by

$$
L:(x, m, \alpha, p) \mapsto L(x, m, \alpha, p)=f(x, m, \alpha)+\langle b(x, m, \alpha), p\rangle .
$$

In the sequel, we will assume that the running cost $f$ and the drift $b$ are such that $H$ is well-defined, $\mathcal{C}^{1}$ with respect to $(x, p)$, and strictly convex with respect to $p$. We will write $\partial_{p} H$ for the partial derivative of $H$ with respect to the $p$ variable.

In the optimal control problem faced by an infinitesimal agent, the flow of population densities is given. From standard optimal control theory (for example using dynamic programming), the best strategy can be characterized through the value function $u$ of the above optimal control problem for a typical agent, which satisfies a Hamilton-Jacobi-Bellman (HJB) equation. Together with the equilibrium condition on the distribution, we obtain that the equilibrium best response $\hat{\alpha}$ is characterized by

$$
\hat{\alpha}(t, x)=\underset{\alpha \in \mathbb{R}^{k}}{\arg \max }\{-L(x, m(t, \cdot), \alpha, \nabla u(t, x))\},
$$


where $(u, m)$ solves the following forward-backward PDE system:

$$
\begin{cases}0=-\frac{\partial u}{\partial t}(t, x)-\nu \Delta u(t, x)+H(x, m(t, \cdot), \nabla u(t, x)), & \text { in }[0, T) \times \mathbb{T}^{d}, \\ 0=\frac{\partial m}{\partial t}(t, x)-\nu \Delta m(t, x) & \\ \quad-\operatorname{div}\left(m(t, \cdot) \partial_{p} H(\cdot, m(t), \nabla u(t, \cdot))(x),\right. & \text { in }(0, T] \times \mathbb{T}^{d}, \\ u(T, x)=g(x, m(T, \cdot)), \quad m(0, x)=m_{0}(x), & \text { in } \mathbb{T}^{d} .\end{cases}
$$

Recall that $\nu=\frac{1}{2} \sigma^{2}$.

EXAMPLE 3.1 ( $f$ depends separately on $\alpha$ and $m$ ). Consider the case where the drift is the control, i.e., $b(x, m, \alpha)=\alpha$ with $k=d$, and the running cost is of the form $f(x, m, \alpha)=L_{0}(x, \alpha)+f_{0}(x, m)$ where $L_{0}(x, \cdot): \mathbb{R}^{d} \ni \alpha \mapsto L_{0}(x, \alpha) \in \mathbb{R}$ is strictly convex and such that $\lim _{|\alpha| \rightarrow \infty} \min _{x \in \mathbb{T}^{d}} \frac{L_{0}(x, \alpha)}{|\alpha|}=+\infty$. We set $H_{0}(x, p)=$ $\max _{\alpha \in \mathbb{R}^{d}}\langle-p, \alpha\rangle-L_{0}(x, \alpha)$, which is convex with respect to $p$. Then

$$
H(x, m, p)=\max _{\alpha \in \mathbb{R}^{d}}\left\{-L_{0}(x, \alpha)-\langle\alpha, p\rangle\right\}-f_{0}(x, m)=H_{0}(x, p)-f_{0}(x, m) .
$$

EXAmPle 3.2 (Quadratic Hamiltonian with separate dependence). Consider the setting of Example 3.1 and assume $L_{0}(x, \alpha)=\frac{1}{2}|\alpha|^{2}$, i.e., $H_{0}(x, \cdot)=H_{0}^{*}(x, \cdot)=$ $\frac{1}{2}|\cdot|^{2}$, where $H_{0}^{*}$ denotes the convex conjugate of $H_{0}$ with respect to the second variable. Then the maximizer in (3.4) is $-p$, the Hamiltonian reads $H(x, m, p)=$ $\frac{1}{2}|p|^{2}-f_{0}(x, m)$ and the equilibrium best response is $\hat{\alpha}(t, x)=-\nabla u(t, x)$ where $(u, m)$ solves the PDE system

$$
\begin{cases}-\frac{\partial u}{\partial t}(t, x)-\nu \Delta u(t, x)+\frac{1}{2}|\nabla u(t, x)|^{2}=f_{0}(x, m(t, \cdot)), & \text { in }[0, T) \times \mathbb{T}^{d} \\ \frac{\partial m}{\partial t}(t, x)-\nu \Delta m(t, x)-\operatorname{div}(m(t, \cdot) \nabla u(t, \cdot))(x)=0, & \text { in }(0, T] \times \mathbb{T}^{d} \\ u(T, x)=g(x, m(T, \cdot)), \quad m(0, x)=m_{0}(x), & \text { in } \mathbb{T}^{d}\end{cases}
$$

Note that in the first equation, the coupling is only through the source term in the right hand side.

Mean field type control problem. In the MFC problem, the value function is not a function of $(t, x)$ only. Indeed, from the definition (1.3) 1.4 one can see that changing the control $\alpha$ also changes the density $m^{\alpha}$ (which is not the case in the optimal control problem faced by an infinitesimal agent in a MFG, see (1.1)). Hence the value function of the central planner is a function of the population's distribution and dynamic programming arguments have been developed using this point of view 94, 95, 31, 105, 26, 65. However, it is still possible to characterize the solution through a system of PDEs over the finite dimensional space $\mathcal{Q}_{T}=$ $[0, T] \times \mathcal{Q}$. This system can be obtained either from the value function or via calculus of variation (see e.g. [30. Chapter 4] for more details on the latter approach). A necessary condition for the existence of a smooth feedback function $\alpha^{*}$ achieving $J^{M F C}\left(\alpha^{*}\right)=\min J^{M F C}(\alpha)$ is that

$$
\alpha^{*}(t, x)=\underset{\alpha \in \mathbb{R}^{k}}{\arg \max }\{-L(x, m(t, \cdot), \alpha, \nabla u(t, x))\},
$$


where $(u, m)$ solve the following system of partial differential equations

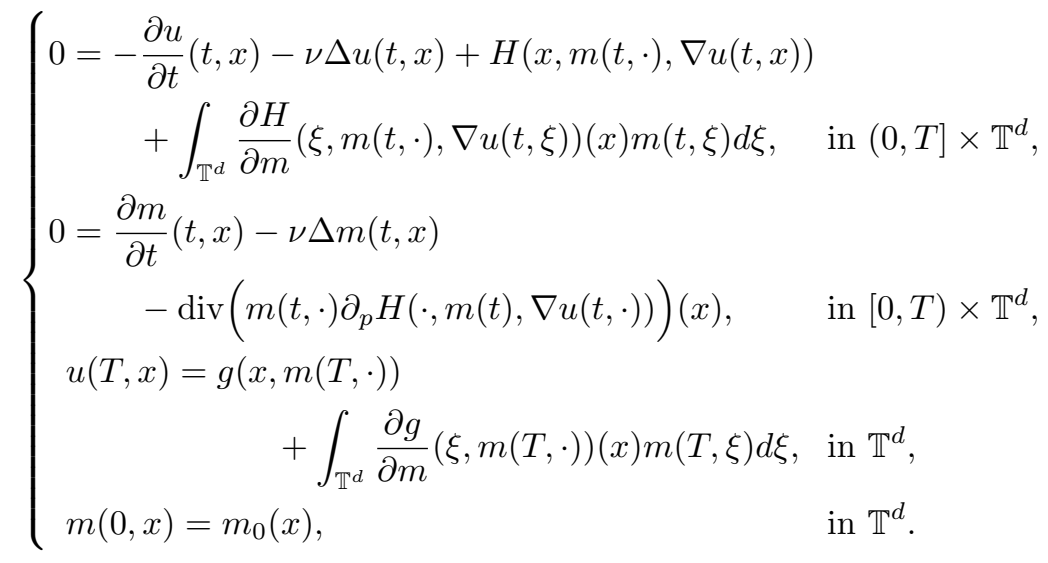

Compared with the MFG system (3.11), there are extra terms involving the partial derivatives with respect to $m$, which should be understood in the following sense: if $\varphi: L^{2}\left(\mathbb{R}^{d}\right) \rightarrow \mathbb{R}$ is differentiable,

$$
\frac{d}{d \varepsilon} \varphi(m+\varepsilon \tilde{m})(x)_{\mid \varepsilon=0}=\int_{\mathbb{R}^{d}} \frac{\partial \varphi}{\partial m}(m)(\xi) \tilde{m}(\xi) d \xi .
$$

See [30, Chapter 4] for more details. If the cost functions and the drift function depends on the density only locally (i.e., only on the density at the current position of the agent), $\frac{\partial}{\partial m}$ becomes a derivative in the usual sense.

Existence and uniqueness. The interested reader is referred to e.g. [96, 93, 41 for details on the question of existence and uniqueness of solutions for the MFG PDE system, and to e.g. 7, 8, for the corresponding MFC system. We simply mention here that the existence of solutions can be typically be obtained when the mean-field interactions are local and smooth or occurring through a regularizing kernel as explained in 93 . As for uniqueness, in the MFG setting, a general sufficient condition for uniqueness is the so-called Lasry-Lions monotonicity condition 93 . Intuitively, it holds when the cost function discourages the players from gathering, i.e., from having a density taking large values. More precisely, when considering a local dependence on the distribution and when the terminal cost $g$ does not depend on $m$, a sufficient condition for uniqueness of a classical solution the MFG PDE system is the positive definiteness of the matrix:

$$
\left(\begin{array}{cc}
-\frac{2}{m} \frac{\partial H}{\partial m}(x, m, p) & \frac{\partial}{\partial m} \partial_{p}^{T} H(x, m, p) \\
\frac{\partial}{\partial m} \partial_{p} H(x, m, p) & 2 D_{p, p}^{2} H(x, m, p)
\end{array}\right)
$$

for all $x \in \mathbb{T}^{d}, m>0$ and $p \in \mathbb{R}^{d}$. If $H$ depends separately on $p$ and $m$, then $\frac{\partial}{\partial m} \partial_{p} H(x, m, p)=0$ and the condition for MFG becomes: $H$ is strictly convex with respect to $p$ for $m>0$ and non-increasing with respect to $m$, or $H$ is convex with respect to $p$ and strictly decreasing with respect to $m$.

As for the MFC problem, an analogous condition has been proposed in [7, when the mean field interactions are of local type. Letting $\mathcal{H}(x, m, p)=m H(x, m, p)$, a sufficient condition is that: for every $x \in \mathbb{T}^{d}$ and $m>0, p \mapsto \mathcal{H}(x, m, p)$ is strictly convex, and for every $x \in \mathbb{T}^{d}$ and $p \in \mathbb{R}^{d}, \mathbb{R}_{+} \ni m \mapsto \mathcal{H}(x, m, p)$ is strictly concave. 
An interesting example in which the Lasry-Lions monotonicity condition can be understood quite intuitively is crowd motion with congestion. We come back to this point below in $\S 4.4$.

3.2. A Finite difference scheme. In this section, we present a finite-difference scheme first introduced in [4]; see also [1]. We consider the special case described in Example 3.1 and we focus on the case of local interactions, so that $H(x, m, p)=H_{0}(x, p)-f_{0}(x, m)$ for all $x \in \mathbb{R}^{d}, m \in[0,+\infty), p \in \mathbb{R}^{d}$. Similar methods have been applied and at least partially analyzed in situations when the Hamiltonian does not depend separately on $m$ and $p$ (for example models addressing congestion, see e.g. [6]).

To alleviate the notation, we present the scheme in the one-dimensional setting, i.e., $d=1$, so the domain is the one-dimensional unit torus, denoted by $\mathbb{T}$.

Discretization. Let $N_{T}$ and $N_{h}$ be two positive integers. We consider $\left(N_{T}+1\right)$ and $\left(N_{h}+1\right)$ points in time and space respectively. For any integers $i<j$, let $\llbracket i, j \rrbracket=\{i, \ldots, j\}$ and $\llbracket j \rrbracket=\llbracket 0, j \rrbracket$. Let $\Delta t=T / N_{T}$ and $h=1 / N_{h}$, and $t_{n}=$ $n \times \Delta t, x_{i}=i \times h$ for $(n, i) \in \llbracket N_{T} \rrbracket \times \llbracket N_{h} \rrbracket$.

We approximate $u$ and $m$ respectively by vectors $U$ and $M \in \mathbb{R}^{\left(N_{T}+1\right) \times\left(N_{h}+1\right)}$, that is, $u\left(t_{n}, x_{i}\right) \approx U_{i}^{n}$ and $m\left(t_{n}, x_{i}\right) \approx M_{i}^{n}$ for each $(n, i) \in \llbracket N_{T} \rrbracket \times \llbracket N_{h} \rrbracket$. We use a superscript and a subscript respectively for the time and space indices. Since we consider periodic boundary conditions, we slightly abuse notation and for any $W \in \mathbb{R}^{N_{h}+1}$, we identify $W_{N_{h}+1}$ with $W_{1}$, and $W_{-1}$ with $W_{N_{h}-1}$. The periodic boundary condition will be translated into the constraint $W_{N_{h}}=W_{0}$.

We introduce the finite difference operators

$$
\begin{aligned}
& \left(D_{t} W\right)^{n}=\frac{1}{\Delta t}\left(W^{n+1}-W^{n}\right), \quad n \in \llbracket N_{T}-1 \rrbracket, \quad W \in \mathbb{R}^{N_{T}+1}, \\
& (D W)_{i}=\frac{1}{h}\left(W_{i+1}-W_{i}\right), \quad i \in \llbracket N_{h} \rrbracket, \quad W \in \mathbb{R}^{N_{h}+1}, \\
& \left(\Delta_{h} W\right)_{i}=-\frac{1}{h^{2}}\left(2 W_{i}-W_{i+1}-W_{i-1}\right), \quad i \in \llbracket N_{h} \rrbracket, \quad W \in \mathbb{R}^{N_{h}+1}, \\
& {\left[\nabla_{h} W\right]_{i}=\left((D W)_{i},(D W)_{i-1}\right)^{\top}, \quad i \in \llbracket N_{h} \rrbracket, \quad W \in \mathbb{R}^{N_{h}+1} \text {. }}
\end{aligned}
$$

Discrete Hamiltonian. Let $\tilde{H}_{0}: \mathbb{T} \times \mathbb{R} \times \mathbb{R} \rightarrow \mathbb{R},\left(x, p_{1}, p_{2}\right) \mapsto \tilde{H}_{0}\left(x, p_{1}, p_{2}\right)$ be a discrete Hamiltonian, assumed to satisfy the following properties:

( $\mathrm{H} 1)$ Monotonicity: for every $x \in \mathbb{T}, \tilde{H}_{0}$ is nonincreasing in $p_{1}$ and nondecreasing in $p_{2}$.

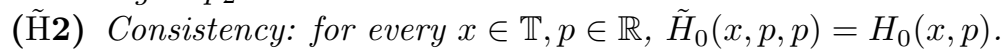



( $\tilde{\mathrm{H}} 4)$ Convexity: for every $x \in \mathbb{T},\left(p_{1}, p_{2}\right) \mapsto \tilde{H}_{0}\left(x, p_{1}, p_{2}\right)$ is convex.

We refer to [4, 1] for more details.

EXAMPLE 3.3. For instance, if $H_{0}(x, p)=\frac{1}{2}|p|^{2}$, then one can take $\tilde{H}_{0}\left(x, p_{1}, p_{2}\right)=$ $\frac{1}{2}\left|P_{K}\left(p_{1}, p_{2}\right)\right|^{2}$ where $P_{K}$ denotes the projection on $K=\mathbb{R}_{-} \times \mathbb{R}_{+}$.

REMARK 3.4. Analogously, for $d$-dimensional problems, the discrete Hamiltonians that we consider are real valued functions defined on $\mathbb{T}^{d} \times\left(\mathbb{R}^{d}\right)^{2}$. 
Discrete HJB equation. We consider the following discrete version of the HJB equation 3.3a):

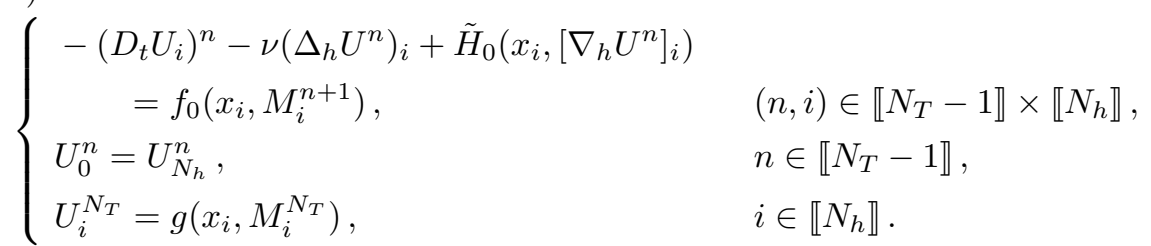

Note that it is an implicit scheme since the equation is backward in time.

Discrete KFP equation. To define an appropriate discretization of the KFP equation $(3.3 \mathrm{~b})$, we start by considering the weak form. For a smooth test function $w \in \mathcal{C}^{\infty}([0, T] \times \mathbb{T})$, it involves, among other terms, the expression

$$
\begin{aligned}
& -\int_{\mathbb{T}} \partial_{x}\left(\partial_{p} H_{0}\left(x, \partial_{x} u(t, x)\right) m(t, x)\right) w(t, x) d x \\
& =\int_{\mathbb{T}} \partial_{p} H_{0}\left(x, \partial_{x} u(t, x)\right) m(t, x) \partial_{x} w(t, x) d x,
\end{aligned}
$$

where we used an integration by parts and the periodic boundary conditions. In view of what precedes, it is quite natural to propose the following discrete version of the right hand side of (3.8):

$h \sum_{i=0}^{N_{h}-1} M_{i}^{n+1}\left(\partial_{p_{1}} \tilde{H}_{0}\left(x_{i},\left[\nabla_{h} U^{n}\right]_{i}\right) \frac{W_{i+1}^{n}-W_{i}^{n}}{h}+\partial_{p_{2}} \tilde{H}_{0}\left(x_{i},\left[\nabla_{h} U^{n}\right]_{i}\right) \frac{W_{i}^{n}-W_{i-1}^{n}}{h}\right)$.

Performing a discrete integration by parts, we obtain the discrete counterpart of the left hand side of 3.8 as follows: $-h \sum_{i=0}^{N_{h}-1} \mathcal{T}_{i}\left(U^{n}, M^{n+1}\right) W_{i}^{n}$, where $\mathcal{T}_{i}$ is the following discrete transport operator:

$$
\begin{aligned}
\mathcal{T}_{i}(U, M)=\frac{1}{h}( & M_{i} \partial_{p_{1}} \tilde{H}_{0}\left(x_{i},\left[\nabla_{h} U\right]_{i}\right)-M_{i-1} \partial_{p_{1}} \tilde{H}_{0}\left(x_{i-1},\left[\nabla_{h} U\right]_{i-1}\right) \\
& \left.+M_{i+1} \partial_{p_{2}} \tilde{H}_{0}\left(x_{i+1},\left[\nabla_{h} U\right]_{i+1}\right)-M_{i} \partial_{p_{2}} \tilde{H}_{0}\left(x_{i},\left[\nabla_{h} U\right]_{i}\right)\right) .
\end{aligned}
$$

Then, for the discrete version of 3.3b, we consider:

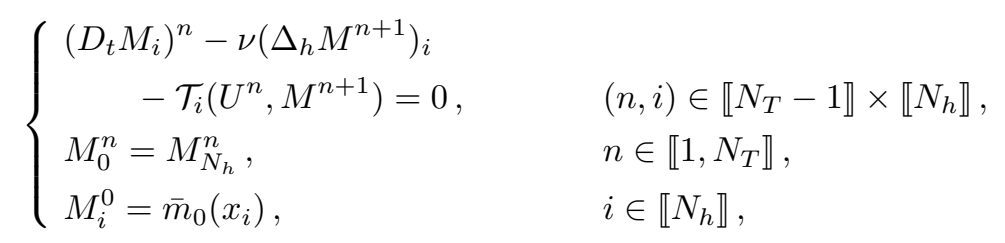

where,

$$
\bar{m}_{0}\left(x_{i}\right)=\int_{\left|x-x_{i}\right| \leq h / 2} m_{0}(x) d x .
$$

Here again, the scheme is implicit since the equation is forward in time. 
REMARK 3.5 (Structure of the discrete system). The finite difference system (3.7)- 3.9) preserves the structure of the PDE system (3.11) in the following sense: The operator $M \mapsto-\nu\left(\Delta_{h} M\right)_{i}-\mathcal{T}_{i}(U, M)$ is the adjoint of the linearization of the operator $U \mapsto-\nu\left(\Delta_{h} U\right)_{i}+\tilde{H}_{0}\left(x_{i},\left[\nabla_{h} U\right]_{i}\right)$. Indeed,

$$
\sum_{i} \mathcal{T}_{i}(U, M) W_{i}=-\sum_{i} M_{i}\left\langle\partial_{p} \tilde{H}_{0}\left(x_{i},\left[\nabla_{h} U\right]_{i}\right),\left[\nabla_{h} W\right]_{i}\right\rangle .
$$

Convergence results. Existence and uniqueness for the discrete system have been proved in [4, Theorems 6 and 7]. The monotonicity properties ensure that the grid function $M$ is nonnegative. By construction of $\mathcal{T}$, the scheme preserves the total mass $h \sum_{i=0}^{N_{h}-1} M_{i}^{n}$. Note that there is no restriction on the time step since the scheme is implicit. Thus, this method may be used for long horizons and the scheme can be very naturally adapted to ergodic MFGs, see 4 .

Furthermore, convergence results are available. A first type of convergence theorems see [4, 2, 3] (in particular [4, Theorem 8] for finite horizon problems) make the assumption that the MFG system of PDEs has a unique classical solution and strong versions of Lasry-Lions monotonicity assumptions, see [91, 92, 93. Under such assumptions, the solution to the discrete system converges towards the classical solution as the grid parameters $h$ and $\Delta t$ tend to zero. Another type of results, obtained in [13, is the convergence of the solution of the discrete problem to weak solutions of the system of forward-backward PDEs. We refer to $\mathbf{1 3}$ for the precise statement but let us stress that these results have been proved without assuming the existence of a (weak) solution to the MFG PDE system, nor LasryLions monotonicity assumptions. This approach can thus be seen as an alternative proof of existence of weak solutions of the MFG PDE system. Besides the setting presented here, similar finite difference schemes have been developed for mean field games with interaction through the law of the controls [5] or in a time-fractional setting $[39$.

3.3. A Semi-Lagrangian scheme. In this section, we present an alternative numerical scheme which relies on a Lagrangian viewpoint instead of a Eulerian point of view as in the aforementioned finite difference scheme. Intuitively, the Lagrangian approach corresponds to idea of following the dynamics of typical player. In [4, 48, 49] Carlini and Silva have developed a semi-Lagrangian scheme for MFG in which the diffusion term can be absent or degenerate. For ease of presentation, take $d=1, \mathcal{Q}=\mathbb{R}$, and let us focus on the case without viscosity (first order setting). The Lagrangian point of view is particularly relevant in this situation, because in the absence of noise, a trajectory is completely determined by the initial position and the control. More precisely, if $b(x, m, \alpha)=\alpha$ and $\nu=0$, then the solution of the state equation 1.2 is given by

$$
X_{t}^{\alpha}=X_{0}^{\alpha}+\int_{0}^{t} \alpha\left(s, X_{s}^{\alpha}\right) d s, \quad t \geq 0 .
$$

Taking, as in Example 3.2 , a running cost function of the form $f(x, m, \alpha)=\frac{1}{2}|\alpha|^{2}+$ $f_{0}(x, m)$ and a terminal cost function $g(x, m)$ (where $f_{0}$ and $g$ depend on $m \in L^{2}(\mathcal{Q})$ 
in a potentially non-local way) leads to the following MFG PDE system:

$$
\begin{cases}-\frac{\partial u}{\partial t}(t, x)+\frac{1}{2}|\nabla u(t, x)|^{2}=f_{0}(x, m(t, \cdot)), & \text { in }[0, T) \times \mathbb{R}, \\ \frac{\partial m}{\partial t}(t, x)-\operatorname{div}(m(t, \cdot) \nabla u(t, \cdot))(x)=0, & \text { in }(0, T] \times \mathbb{R}, \\ u(T, x)=g(x, m(T, \cdot)), \quad m(0, x)=m_{0}(x), & \text { in } \mathbb{R} .\end{cases}
$$

This amounts to taking $\nu=0$ (and changing the domain) in system (3.5).

Discrete HJB equation. Given a flow of densities $m=\left(m_{t}\right)_{t \in[0, T]}$, the corresponding value function $u$ admits the following representation formula:

$$
u(t, x)=\inf _{\alpha \in L^{2}([t, T] ; \mathbb{R})} \int_{t}^{T}\left[\frac{1}{2}|\alpha(s)|^{2}+f_{0}\left(X_{s}^{\alpha, t, x}, m(s, \cdot)\right)\right] d s+g\left(X_{T}^{\alpha, t, x}, m(T, \cdot)\right),
$$

where $X^{\alpha, t, x}$ starts from $x$ at time $t$ and is controlled by $\alpha$.

Based on this intuition, let us consider the equation:

$$
\begin{cases}U_{i}^{n}=S_{\Delta t, h}[m]\left(U^{n+1}, i, n\right), & (n, i) \in \llbracket N_{T}-1 \rrbracket \times \mathbb{Z}, \\ U_{i}^{N_{T}}=g\left(x_{i}, m(T, \cdot)\right), & i \in \mathbb{Z},\end{cases}
$$

where $S_{\Delta t, h}$ is defined as

$$
S_{\Delta t, h}[m](W, n, i)=\inf _{\alpha \in \mathbb{R}^{d}}\left\{I[W]\left(x_{i}+\alpha \Delta t\right)+\frac{1}{2}|\alpha|^{2} \Delta t\right\}+f_{0}\left(x_{i}, m\left(t_{n}, \cdot\right)\right) \Delta t,
$$

with $I: \mathcal{B}(\mathbb{Z}) \rightarrow \mathcal{C}_{b}(\mathbb{R})$ denoting the interpolation operator defined as

$$
I[W](\cdot)=\sum_{i \in \mathbb{Z}} W_{i} \beta_{i}(\cdot),
$$

where $\mathcal{B}(\mathbb{Z})$ is the set of bounded functions from $\mathbb{Z}$ to $\mathbb{R}$, and $\beta_{i}=\left[1-\frac{\left|x-x_{i}\right|}{h}\right]_{+}$is the triangular function with support $\left[x_{i-1}, x_{i+1}\right]$ and such that $\beta_{i}\left(x_{i}\right)=1$.

From the solution $U=\left(U_{i}^{n}\right)_{n, i}$ of the discrete HJB equation (3.12) for a given density flow $m$, we interpolate it to construct the following function $u_{\Delta t, h}[m](x, t)$ : $[0, T] \times \mathbb{R} \rightarrow \mathbb{R}$,

$$
u_{\Delta t, h}[m](t, x)=I\left[U^{\left[\frac{t}{\Delta t}\right]}\right](x), \quad(t, x) \in[0, T] \times \mathbb{R} .
$$

Discrete KFP equation. In order to write a discrete version of the KFP equation, the solution of the discrete HJB equation is replaced by a regularized version. Let $\rho \in \mathcal{C}_{c}^{\infty}(\mathbb{R})$ with $\rho \geq 0$ and $\int \rho(x) d x=1$. For $\epsilon>0$, let us consider the mollifier $\rho_{\epsilon}(x)=\frac{1}{\epsilon} \rho(x / \epsilon)$ and define

$$
u_{\Delta t, h}^{\epsilon}[m](t, \cdot)=\rho_{\epsilon} * u_{\Delta t, h}[m](t, \cdot), \quad t \in[0, T] .
$$

Then let us introduce, for $(t, x) \in[0, T] \times \mathbb{R}$, the induced control

$$
\hat{\alpha}_{\Delta t, h}^{\epsilon}[m](t, x)=-\nabla u_{\Delta t, h}^{\epsilon}[m](t, x),
$$

and its discrete counter part: for $(n, i) \in \llbracket N_{T} \rrbracket \times \mathbb{Z}$,

$$
\hat{\alpha}_{n, i}^{\epsilon}=\hat{\alpha}_{\Delta t, h}^{\epsilon}[m]\left(t_{n}, x_{i}\right) .
$$

Then define the following discrete flow: for $(n, i) \in \llbracket N_{T}-1 \rrbracket \times \mathbb{Z}$,

$$
\Phi_{n, n+1, i}^{\epsilon}[m]=x_{i}+\hat{\alpha}_{\Delta t, h}^{\epsilon}[m]\left(t_{n}, x_{i}\right) \Delta t .
$$


We can now introduce the discrete KFP equation for $M^{\epsilon}[m]=\left(M_{i}^{\epsilon, n}[m]\right)_{n, i}$ :

$$
\begin{cases}M_{i}^{\epsilon, n+1}[m]=\sum_{j} \beta_{i}\left(\Phi_{n, n+1, j}^{\epsilon}[m]\right) M_{j}^{\epsilon, n}[m], & (n, i) \in \llbracket N_{T}-1 \rrbracket \times \mathbb{Z}, \\ M_{i}^{\epsilon, 0}[m]=\int_{\left[x_{i}-h / 2, x_{i}+h / 2\right]} m_{0}(x) d x, & i \in \mathbb{Z} .\end{cases}
$$

Intuitively, the idea is as depicted by Figure 8 First, if control $\left(\alpha_{i}^{n}\right)_{i, n}$, an agent starting at time $n$ at point $x_{i}$ is supposed to arrive at time $n+1$ at point $x_{i}+\alpha_{i}^{n} \Delta t$ (dotted arrow in fuschia). Supposing that this point is between the grid points $x_{j-1}$ and $x_{j}$, the mass $M_{i}^{n}$ (rectangle in blue) at $x_{i}$ is going to be split between $x_{j-1}$ and $x_{j}$. The proportion of mass moving to each point is proportional to the values of the hat functions $\beta_{j-1}$ and $\beta_{j}$ (in red) at the arrival point $x_{i}+\alpha_{i}^{n} \Delta t$.

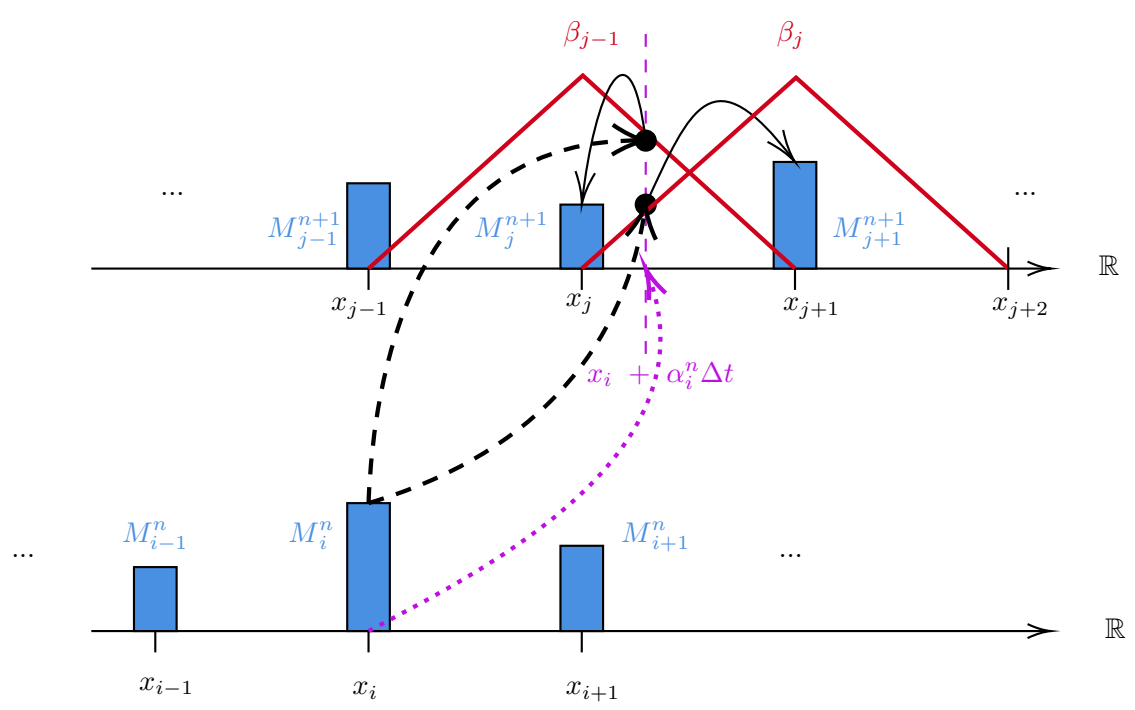

FiguRE 8. Illustration of the evolution of the mass as given by (3.16) and explained in the text. The bottom row corresponds to time $n$ and the top row to time $n+1$.

From here, we can recover a function by defining $m_{\Delta t, h}^{\epsilon}[m]:[0, T] \times \mathbb{R} \rightarrow \mathbb{R}$ as: for $n \in \llbracket N_{T}-1 \rrbracket$, for $t \in\left[t_{n}, t_{n+1}\right)$,

$$
\begin{aligned}
m_{\Delta t, h}^{\epsilon}[m](t, x)=\frac{1}{\rho}\left[\frac{t_{n+1}-t}{\Delta t} \sum_{i \in \mathbb{Z}} M_{i}^{\epsilon, n}[m] \mathbf{1}_{\left[x_{i}-h / 2, x_{i}+h / 2\right]}(x)\right. \\
\left.+\frac{t-t_{n}}{\Delta t} \sum_{i \in \mathbb{Z}} M_{i}^{\epsilon, n+1}[m] \mathbf{1}_{\left[x_{i}-h / 2, x_{i}+h / 2\right]}(x)\right] .
\end{aligned}
$$

The goal is then to solve the following fixed-point problem: Find $\hat{M}$ such that

$$
\hat{M}_{i}^{n}=M_{i}^{n}\left[m_{\Delta t, h}^{\epsilon}[\hat{M}]\right] .
$$

Recall that $\left(M_{i}^{n}\right)_{i, n}$ depends on $\hat{\alpha}_{\Delta t, h}^{\epsilon}$, which itself depends on $\left(U_{i}^{n}\right)_{i, n}$. Hence, as in the finite difference scheme, the equations for the distribution and the value function are coupled. Convergence of the scheme towards the continuous solution of the PDE system has been proved under suitable conditions even in the first order 
$(\nu=0)$ as in the present discussion or degenerate case, see 47, 48, 49, for the details. 


\section{Algorithms to solve the discrete schemes}

In this section, we review several algorithms to solve the numerical schemes introduced in the previous section. Their forward-backward structure is the main challenge. We revisit the methods presented in Section 2, namely, fixed point iterations and Newton iterations.

4.1. Picard (fixed point) iterations. Probably the most straightforward method is to iteratively solve the (discrete) HJB equation and the (discrete) FP equation in order to update respectively the estimate of the value function and the density, using in each equation the most recent estimate of the other function. As presented in Section 2, a damping coefficient can be introduced, which can even account for fictitious play type updates. This approach can be used with the finite difference scheme or the semi-Lagrangian scheme presented earlier in Section 3. We illustrate it here with the semi-Lagrangian scheme in the spirit of 47 . In this context, the iterations take the form described in Algorithm 3. Note that the step corresponding to 3.13 requires computing an infimum over the controls. In the implementation, we can replace $\mathbb{R}^{d}$ by a bounded set, which is then discretized, so that the infimum is taken over a finite number of values. Instead of fixing a priori the number of iterations, we can use a stopping criterion of the form:

$$
\left\|U^{(\epsilon, \mathrm{k}+1)}-U^{(\epsilon, \mathrm{k})}\right\|<\varepsilon, \quad \text { and } \quad\left\|M^{(\epsilon, \mathrm{k}+1)}-M^{(\epsilon, \mathrm{k})}\right\|<\varepsilon,
$$

for some threshold $\varepsilon>0$.

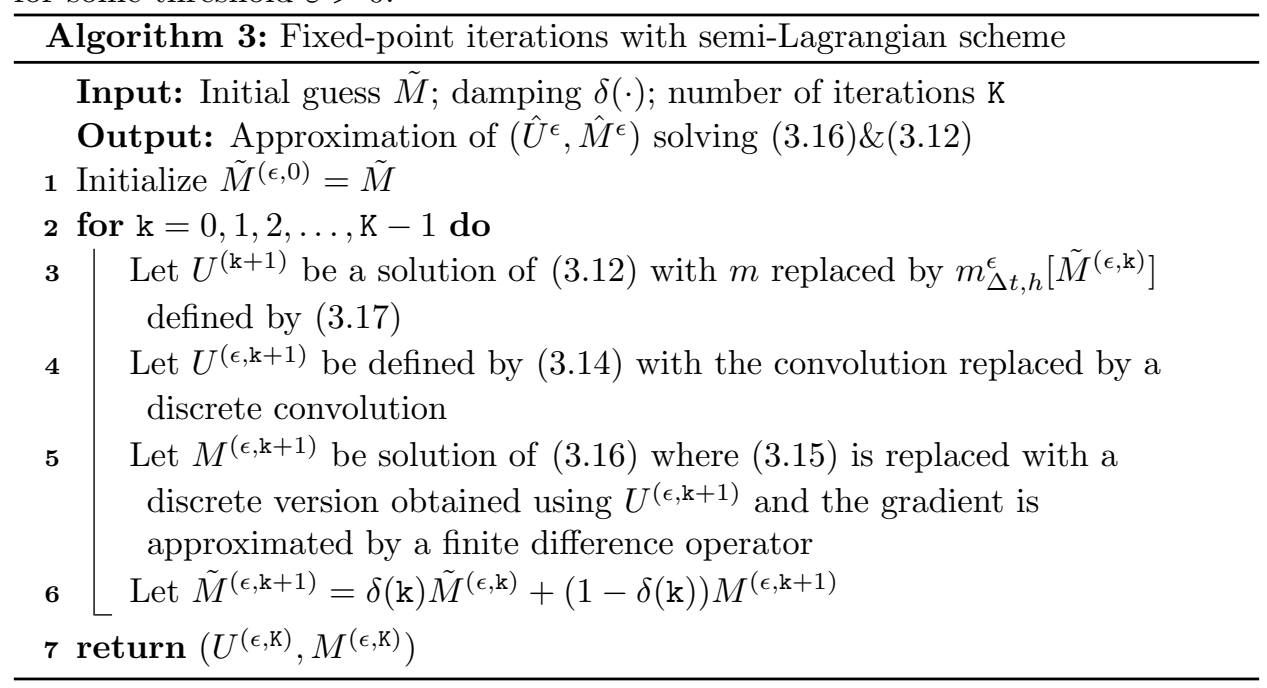

4.2. Numerical illustration: an example with concentration of mass. We borrow an example from [47. There is no terminal cost, i.e., $g \equiv 0$, and the running cost is:

$$
f(x, m, \alpha)=\frac{1}{2}|\alpha|^{2}+\left(x-c^{*}\right)^{2}+\kappa_{M F} V(x, m),
$$

where $c^{*} \in \mathbb{R}$ plays the role of a target position, $\kappa_{M F} \geq 0$ is a parameter for the strength of the mean-field interactions. The first term penalizes large velocity, the second term penalizes being far from the target, and the last term involves meanfield interactions. $V$ is a non-local interaction term which penalizes having a high 

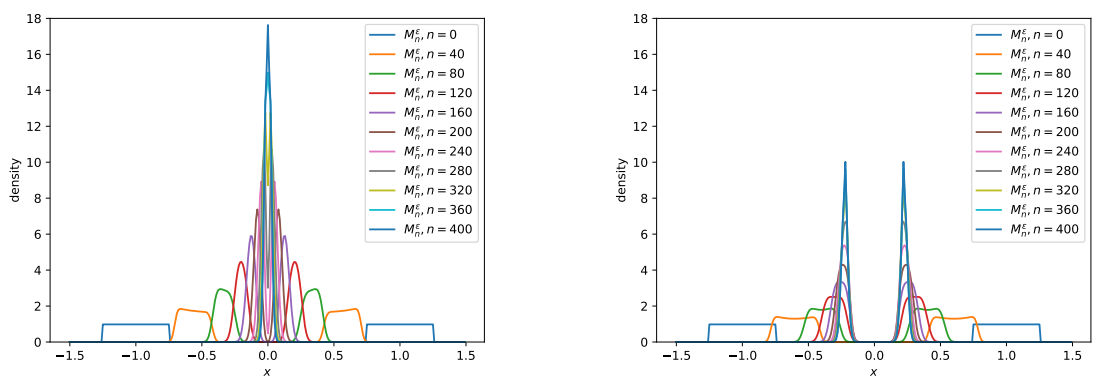

Figure 9. Evolution of the distribution for the example solved with the semi-Lagrangian scheme. Left: $\kappa_{M F}=0.5$; right: $\kappa_{M F}=$ 0.9 .

density around the position $x$, defined by:

$$
V(x, m)=\rho_{\sigma_{V}} *\left(\rho_{\sigma_{V}} * m\right)(x),
$$

where we recall that $\rho_{\sigma_{V}}$ is a mollifier and $*$ denotes the convolution. For the numerical implementation, we used $c^{*}=0, \sigma_{V}=0.25$. The initial distribution is composed of two uniform parts: on $[-1.25,-0.75]$ and on $[0.75,1.25]$. In Figure 9, we compare the evolution of the density for two values of $\kappa_{M F}$ : 0.5 or 0.9 . In the first case, the mass concentrates around $c^{*}=0$. In the second case, due to the higher penalty, the mass does not concentrate at 0 and the two parts of the density remain separate, with lower peaks. We used $T=4$ with 400 time steps and the domain $[-2.5,2.5]$, although for the sake of clarity we show the results for the truncated domain $[-1.5,1.5]$. It can be noticed that at each time step the density remains zero on most of the domain, which is due to the fact that there is no diffusion in this model (i.e., $\nu=0$ in our notation). 
4.3. Newton method. Another solution strategy, proposed by Achdou et al. [2], consists in using Newton method to solve the finite-difference system $(3.7) \&(3.9)$. The main idea is to directly look for a zero of the function

$$
\varphi=\left(\varphi_{\mathcal{U}}, \varphi_{\mathcal{M}}\right)^{\top}
$$

with $\varphi_{\mathcal{U}}$ and $\varphi_{\mathcal{M}}$ defined such that, for $U$ and $M \in \mathbb{R}^{\left(N_{T}+1\right) \times\left(N_{h}+1\right)}$,

$$
\begin{cases}\varphi_{\mathcal{U}}(U, M)=0 & \Leftrightarrow(U, M) \text { solves discrete HJB equation 3.7) } \\ \varphi_{\mathcal{M}}(U, M)=0 & \Leftrightarrow(U, M) \text { solves discrete KFP equation } 3.9 .\end{cases}
$$

Let $J_{\varphi}$ denote the Jacobian of $\varphi$. Newton method then consists in starting from an initial guess $\left(U^{0}, M^{0}\right)$ and iteratively computing $\left(U^{(\mathrm{k}+1)}, M^{(\mathrm{k}+1)}\right)$ given by:

$$
\left(U^{(\mathrm{k}+1)}, M^{(\mathrm{k}+1)}\right)^{\top}=\left(U^{(\mathrm{k})}, M^{(\mathrm{k})}\right)^{\top}-J_{\varphi}\left(U^{(\mathrm{k})}, M^{(\mathrm{k})}\right)^{-1} \varphi\left(U^{(\mathrm{k})}, M^{(\mathrm{k})}\right)
$$

or rather solving for $\left(\tilde{U}^{(\mathrm{k}+1)}, \tilde{M}^{(\mathrm{k}+1)}\right)$ :

$$
J_{\varphi}\left(U^{(\mathrm{k})}, M^{(\mathrm{k})}\right)\left(\tilde{U}^{(\mathrm{k}+1)}, \tilde{M}^{(\mathrm{k}+1)}\right)=-\varphi\left(U^{(\mathrm{k})}, M^{(\mathrm{k})}\right),
$$

and then setting $\left(U^{(\mathrm{k}+1)}, M^{(\mathrm{k}+1)}\right)^{\top}=\left(\tilde{U}^{(\mathrm{k}+1)}, \tilde{M}^{(\mathrm{k}+1)}\right)+\left(U^{(\mathrm{k})}, M^{(\mathrm{k})}\right)^{\top}$.

Hence each step amounts to solving a linear system of the form:

$$
\left(\begin{array}{ll}
A_{\mathcal{U}, \mathcal{U}} & A_{\mathcal{U}, \mathcal{M}} \\
A_{\mathcal{M}, \mathcal{U}} & A_{\mathcal{M}, \mathcal{M}}
\end{array}\right)\left(\begin{array}{c}
U \\
M
\end{array}\right)=\left(\begin{array}{c}
G_{\mathcal{U}} \\
G_{\mathcal{M}}
\end{array}\right)
$$

where

$$
\begin{array}{llrl}
A_{\mathcal{U}, \mathcal{M}}(U, M) & =\nabla_{U} \varphi_{\mathcal{M}}(U, M), & & A_{\mathcal{U}, \mathcal{U}}(U, M)=\nabla_{U} \varphi_{\mathcal{U}}(U, M), \\
A_{\mathcal{M}, \mathcal{U}}(U, M) & =\nabla_{M} \varphi_{\mathcal{U}}(U, M), & & A_{\mathcal{M}, \mathcal{M}}(U, M)=\nabla_{M} \varphi_{\mathcal{M}}(U, M) .
\end{array}
$$

For the numerical implementation, we note that $A_{\mathcal{U}, \mathcal{M}}$ and $A_{\mathcal{M}, \mathcal{U}}$ are blockdiagonal, $A_{\mathcal{U}, \mathcal{U}}=A_{\mathcal{M}, \mathcal{M}}^{\top}$, and:

$$
A_{\mathcal{U}, \mathcal{U}}=\left(\begin{array}{ccccc}
D_{1} & -\frac{1}{\Delta t} \operatorname{Id}_{N_{h}} & \cdots & \ldots & 0 \\
0 & D_{2} & \ddots & 0 & \vdots \\
0 & \ddots & \ddots & \ddots & \vdots \\
\vdots & \ddots & \ddots & \ddots & -\frac{1}{\Delta t} \operatorname{Id}_{N_{h}} \\
0 & \ddots & 0 & 0 & D_{N_{T}}
\end{array}\right),
$$

where $D_{n}$ is the matrix corresponding to the discrete operator:

$$
Z=\left(Z_{i, j}\right)_{i, j} \mapsto\left(\frac{1}{\Delta t} Z_{i, j}-\nu\left(\Delta_{h} Z\right)_{i, j}+\left[\nabla_{h} Z\right]_{i, j} \cdot \nabla_{q} H_{h}\left(x_{i, j},\left[\nabla_{h} U^{(\mathrm{k}), n}\right]_{i, j}\right)\right)_{i, j},
$$

which comes from the linearization of the discrete HJB equation (3.7).

We refer to [1, Section 4] and [12 for more details on possible strategies to solve (4.1). We simply stress that, as usual with Newton method, the choice of the initial guess $\left(U^{(0)}, M^{(0)}\right)$ is important. A possible choice is to use the solution to the corresponding ergodic problem (if it is known or if it can be computed easily). Another possibility is to exploit the fact that the method converges more easily when the viscosity coefficient $\nu$ is large. It is thus possible to use a continuation method: we start by solving the problem with a large $\nu$, then use the solution as an initial guess for the problem with a smaller $\nu$, and so on until the desired viscosity coefficient is reached. 

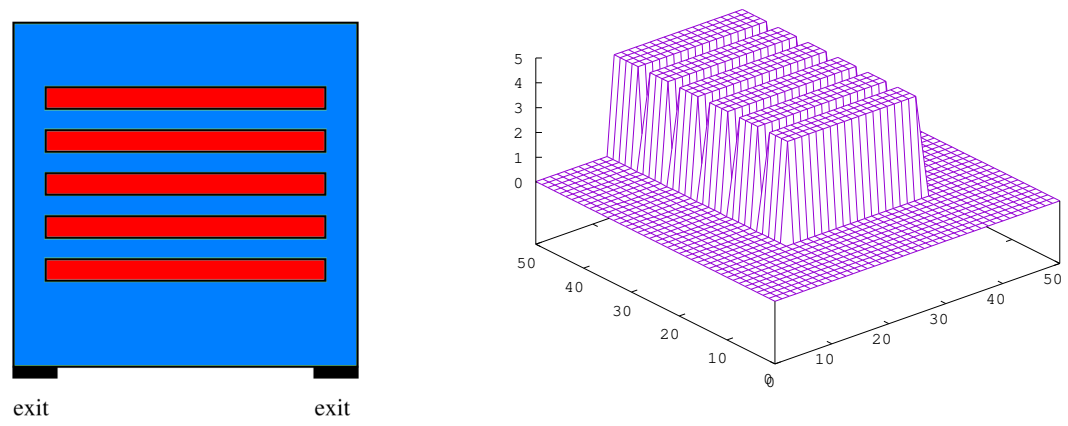

Figure 10. Left: the geometry (obstacles are in red). Right: the density at $t=0$.

4.4. Numerical illustration: evacuation of a room with congestion effects. We provide an example, borrowed from [7, which we solve using the finite difference scheme and Newton method. The model represents a crowd of pedestrians who want to leave a room represented by a square hall (whose side is 50 meters long) containing rectangular obstacles. There are two doors. The chosen geometry and the initial distribution are represented on Figure 10.

For simplicity we focus here on a model with local interactions; see e.g. 89, 6, 14, 8, for other models of this type and [23, 7] for crowd motion models with non-local interactions. Here, the congestion is taken into account through the cost (leading to so-called soft congestion, see Remark 4.1 below): the higher the density at the current position, the higher the price to move. In particular, the running cost does not have a separate dependence in $\alpha$ and $m$ as in Example 3.1. We consider the following Hamiltonian, which depends locally on $m$ and captures congestion effects:

$$
H(x, m, p)=\frac{8|p|^{2}}{(1+m)^{\frac{3}{4}}}-\frac{1}{3200} .
$$

We compare the evolution of the density in the non-cooperative and the cooperative situation:

(1) Mean field games: the MFG PDE system 3.11 becomes

$$
\left\{\begin{array}{c}
-\frac{\partial u}{\partial t}-0.05 \Delta u+\frac{8}{(1+m)^{\frac{3}{4}}}|\nabla u|^{2}=\frac{1}{3200}, \\
\frac{\partial m}{\partial t}-0.05 \Delta m-16 \operatorname{div}\left(\frac{m \nabla u}{(1+m)^{\frac{3}{4}}}\right)=0 .
\end{array}\right.
$$

(2) Mean field control: the KFP equation (4.3b) is the same but the HJB equation becomes

$$
-\frac{\partial u}{\partial t}-0.05 \Delta u+\left(\frac{2}{(1+m)^{\frac{3}{4}}}+\frac{6}{(1+m)^{\frac{7}{4}}}\right)|\nabla u|^{2}=\frac{1}{3200} .
$$

We choose $\nu=0.05$. The time horizon corresponds to $T=50$ minutes and we do not put any terminal cost, meaning $g \equiv 0$. 
The boundary consists of several parts. On the part corresponding to the doors, for $u$, we impose a Dirichlet condition $u=0$, which corresponds to an exit cost. For $m$, we assume that $m=0$ outside the domain, so we also get the Dirichlet condition $m=0$ on this part of the boundary. On the part of the boundary corresponding to the solid walls, for $u$, we impose a homogeneous Neumann boundary condition: $\frac{\partial u}{\partial n}=0$, which means that the velocity of the pedestrians is tangential to the walls. For $m$, we choose the boundary condition: $\nu \frac{\partial m}{\partial n}+m \frac{\partial H}{\partial p}(\cdot, m, \nabla u) \cdot n=0$, therefore $\frac{\partial m}{\partial n}=0$ on this part of the boundary.

The initial density $m_{0}$ is piecewise constant and takes two values 0 and 4 people $/ \mathrm{m}^{2}$, see Figure 10 . At $t=0$, there are 3300 people in the hall.

We use Newton iterations with the finite difference scheme discussed in $\S 3.2$ and originally proposed in [4].

Figure 11 displays the density $m$ for the two models, at $t=1,2,5$ and 15 minutes. In both cases, the pedestrians move between the obstacles towards the narrow corridors leading to the exits, at the left and right sides of the hall. The density thus reaches high values at the intersections of corridors. Then, due to congestion effects, the velocity is lower in the regions where the density is higher. As shown on Figure 11. MFC leads to lower values of density. This is consistent with Figure 12 (left), which shows that MFC leads to a faster exit of the room. Furthermore, the price of anarchy can be estimated numerically. As shown on Figure 12 (right), it is found to increase with respect to the sensitivity to the crowd, captured by the exponent $\frac{3}{4}$ in 4.2 . This coefficient can be replaced by a value $\beta \geq 0$, which parameterizes the congestion effects (i.e., the strength of the mean-field interactions). We conclude that the non-cooperative scenario (MFG) is less and less efficient (relatively to the MFC) when the congestion effects increase. In the extreme case where $\beta=0$, there is no more mean-field interactions, which explains why the PoA is 1 .

REMARK 4.1. The form of soft congestion through the cost function considered here is in contrast with a form of hard congestion taken into account through a density constraint, see e.g. $[\mathbf{9 7}, 4 \mathbf{4 6}$. In the latter case, the density cannot exceed a given threshold, which leads to flat regions in the density surface at points where the constraint is binding, see e.g. [34 for numerical examples. Moreover, a congestion cost is different from a crowd aversion cost represented by an increasing function of $m$ which is in general independent of the control. The latter cost is a penalty term which discourages the agents from being in a crowded region independently of whether they are moving or not. This type of cost typically makes the population spread although not exactly in the same way as a diffusion, see $\S 5.5$ for more details. 

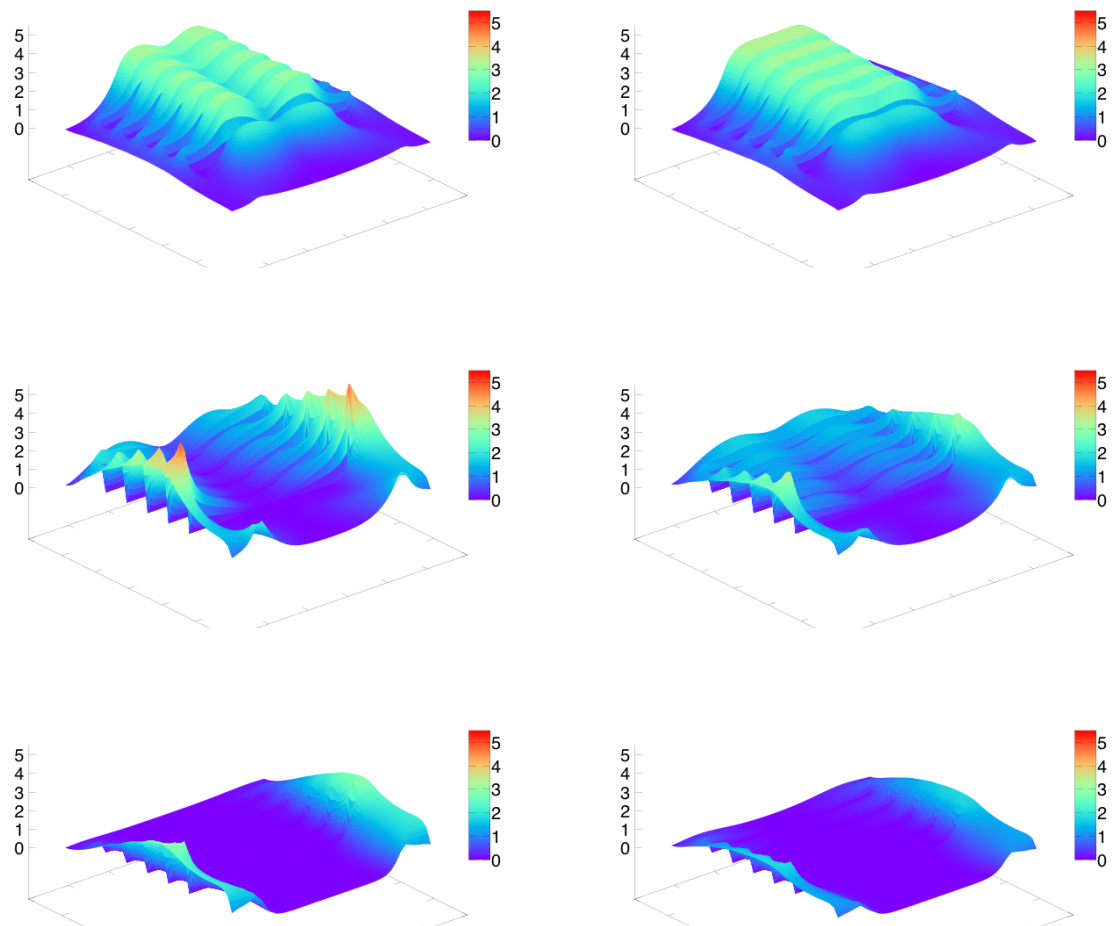

FIGURE 11. The density computed with the two models at different dates, $t=1,5$ and 15 minutes (from top to bottom). Left: Mean field game. Right: Mean field type control.
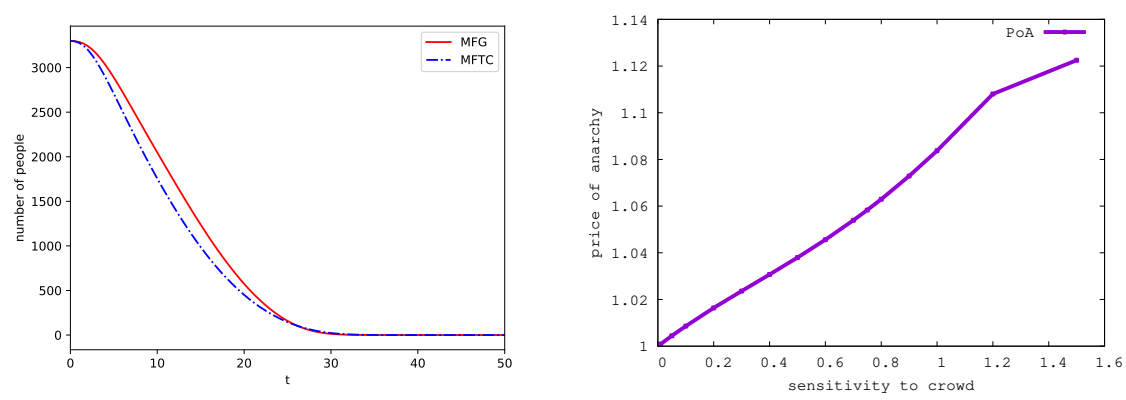

Figure 12. Left: Evolution of the total remaining number of people in the room for the mean field game (red line) and the mean field type control (dashed blue line). Right: Price of Anarchy as a function of the parameter $\beta$. 


\section{Optimization methods for MFC and variational MFGs}

We now turn our attention to mean-field problems for which the PDE system can be interpreted as the optimality condition of a minimization problem for an energy functional subject to a constraint given by a continuity equation. While mean-field control problems can always be formulated in this way, this is not true for all mean-field games. Mean-field games with this property are called variational MFGs. From a theoretical and a numerical viewpoint, the aforementioned formulation as a minimization problem is important because it allows us to solve the problem with optimization techniques, which is not the case in general for fixedpoint problems. In this section, after describing the main ideas of variational MFGs, we focus on the numerical aspects and describe two methods.

5.1. Variational formulation of MFC and MFG. Let us start with the MFC problem (1.3)-1.4. The expectation as an integral against $m^{\alpha}(t, \cdot)$, the probability density of the law of $X_{t}^{\alpha}$, which leads to:

$J^{M F C}(\alpha)=\int_{0}^{T} \int_{\mathcal{Q}} f\left(x, m^{\alpha}(t, \cdot), \alpha(t, x)\right) m^{\alpha}(t, x) d x d t+\int_{\mathcal{Q}} g\left(x, m^{\alpha}(T, \cdot)\right) m^{\alpha}(T, x) d x$.

The cost is thus formulated in a deterministic way and is to be minimized under the constraint given by the KFP equation for $m^{\alpha}$ :

(5.1)

$\frac{\partial m^{\alpha}}{\partial t}(t, x)-\nu \Delta m^{\alpha}(t, x)+\operatorname{div}\left(m^{\alpha}(t, \cdot) b\left(\cdot, m^{\alpha}(t), \alpha(t, \cdot)\right)\right)(x)=0$, in $(0, T] \times \mathcal{Q}_{T}$,

with the initial condition:

$$
m^{\alpha}(0, x)=m_{0}(x), \text { in } \mathcal{Q} .
$$

For a MFG, it is not always possible to characterize the equilibrium flow of densities as the minimizer of a functional. A special class of games for which it is possible is the class of so-called potential mean-field games, namely, games in which the costs $f$ and $g$ derive from a potential. For instance, let us consider the setting where $b$ and $f$ are as in Example 3.2, and suppose in addition that there exists $F_{0}$ and $G$ such that

$$
f_{0}(x, m)=\frac{\delta F_{0}}{\delta m}(x, m), \quad g(x, m)=\frac{\delta G}{\delta m}(x, m) .
$$

Here the derivative should be understood in the sense of measures and we implicitly identify the probability density $m$ with the corresponding probability measure. If we are concerned with square-integrable densities, we can assume that there exist $\mathbf{F}_{0}, \mathbf{G}: \mathcal{P}_{2}(\mathcal{Q}) \rightarrow \mathbb{R}$, where $\mathcal{P}_{2}(\mathcal{Q})$ denotes the set of probability measures on $\mathcal{Q}$ with a second moment, such that:

$\mathbf{F}_{0}(m)-\mathbf{F}_{0}\left(m^{\prime}\right)=\int_{0}^{1} \int_{\mathcal{Q}} f_{0}\left(x,(1-\theta) m+\theta m^{\prime}\right)\left(m-m^{\prime}\right)(d x) d \theta, \quad \forall m, m^{\prime} \in \mathcal{P}_{2}(\mathcal{Q})$,

and likewise for $\mathbf{G}$ and $g$. We can then consider the cost functional

$$
\mathbf{J}(\alpha)=\int_{0}^{T}\left[\int_{\mathcal{Q}} \frac{1}{2}|\alpha(t, x)|^{2} m^{\alpha}(t, x) d x+\mathbf{F}_{0}\left(m^{\alpha}(t, \cdot)\right)\right] d t+\mathbf{G}\left(m^{\alpha}(T, \cdot)\right) .
$$

where, as before, $m^{\alpha}$ solves 5.1 -5.2 with our choice of $b$, namely,

$$
\frac{\partial m^{\alpha}}{\partial t}(t, x)-\nu \Delta m^{\alpha}(t, x)+\operatorname{div}\left(m^{\alpha}(t, \cdot) \alpha(t, \cdot)\right)(x)=0, \text { in }(0, T] \times \mathcal{Q}_{T},
$$


with $m^{\alpha}(0, x)=m_{0}(x)$ in $\mathcal{Q}$. Under suitable conditions, this problem admits a minimizer $\alpha^{*}$ and a corresponding flow of densities $m^{\alpha^{*}}$, from which it is possible to recover the MFG Nash equilibrium $(\hat{m}, \hat{u})$ satisfying the MFG PDE system (3.11).

In the above situation, the variational problem directly stems from the fact that the cost can be interpreted as a potential. More generally, MFG for which the equilibrium can be characterized via a critical point of a variational problem are called variational MFG. In this case, the PDE system can be viewed either as the equilibrium condition of the MFG or as the critical point condition of the variational problem. In the latter problem, the energy functional to be minimized does not necessarily correspond to the original cost functional of an infinitesimal player. We refer the interested reader to e.g. [41, 29] for more details on variational and potential MFGs.

5.2. A PDE driven optimal control problem. To fix ideas, let us consider the following problem: Minimize the function

$\mathcal{B}(m, w)=\int_{0}^{T} \int_{\mathbb{T}^{d}}(\mathfrak{L}(x, m(t, x), w(t, x))+\mathfrak{F}(x, m(t, x))) d x d t+\int_{\mathbb{T}^{d}} \mathfrak{G}(x, m(T, x)) d x$

on pairs $(m, w)$ such that $m \geq 0$ and

$$
\begin{cases}\frac{\partial m}{\partial t}-\nu \Delta m+\operatorname{div} w=0, & \text { in }(0, T] \times \mathbb{T}^{d}, \\ \left.m\right|_{t=0}=m_{0}, & \text { in } \mathbb{T}^{d},\end{cases}
$$

where we use the notation:

$\mathfrak{F}(x, m)=\left\{\begin{array}{ll}\int_{0}^{m} \tilde{f}(x, s) d s, & \text { if } m \geq 0, \\ +\infty, & \text { otherwise, }\end{array} \quad \mathfrak{G}(x, m)= \begin{cases}\int_{0}^{m} \tilde{g}(x, s) d s, & \text { if } m \geq 0, \\ +\infty, & \text { otherwise, }\end{cases}\right.$ and

$$
\mathfrak{L}(x, m, w)= \begin{cases}m \tilde{\ell}\left(x, \frac{w}{m}\right), & \text { if } m>0, \\ 0, & \text { if } m=0 \text { and } w=0, \\ +\infty, & \text { otherwise. }\end{cases}
$$

For simplicity, we assume that for every $x, \tilde{\ell}(x, \cdot)$ is a power-like function with exponent greater than 1 (i.e., it is bounded above and below by $p \mapsto p^{r}$ for some $r>1$, up to multiplicative and additive constants; see e.g. 43 for detailed assumptions). If $\tilde{f}(x, \cdot)$ and $\tilde{g}(x, \cdot)$ are the antiderivatives of $m \mapsto m f_{0}(x, m)$ and $m \mapsto m g(x, m)$ respectively, and if $\tilde{\ell}=L_{0}$, then this problem corresponds to the MFC problem in the setting of Example 3.1 when the cost functions depend only locally on $m$. Problems of the above type can also stem from the variational formulation of some MFG, as originally explained by Lasry and Lions in $\mathbf{9 3}$. We refer the interested read to $[\mathbf{4 4}, 43,8$ and the references therein for a rigorous development of these ideas.

Here $w$ plays the role of the product $m \alpha$. Using this new variable, the continuity equation becomes (5.4), which is linear in $(m, w)$. Under suitable conditions, $\mathcal{B}$ is convex and hence this is a convex minimization problem under a linear constraint. For instance, we typically assume that $\tilde{f}$ and $\tilde{g}$ are nondecreasing, which implies that $\mathfrak{F}$ and $\mathfrak{G}$ are convex, and that $\tilde{\ell}(x, \cdot)$ is a convex power-like function, which implies that $\mathfrak{L}$ is convex with respect to $(m, w)$. 
In this case, the problem admits the following dual formulation: Maximize over $u$ such that $u(T, x)=g(x)$, the function

$$
\mathcal{A}(u)=\inf _{m} \mathcal{A}(u, m)
$$

with:

$$
\begin{aligned}
\mathcal{A}(u, m)= & \int_{0}^{T} \int_{\mathbb{T}} m(t, x)\left(\partial_{t} u(t, x)+\nu \Delta u(t, x)-\mathfrak{H}(x, m(t, x), \nabla u(t, x))\right) d x d t \\
& +\int_{\mathbb{T}} m_{0}(x) u(0, x) d x,
\end{aligned}
$$

where

$$
\mathfrak{H}(x, m, p)=\sup _{\alpha}\{-\mathfrak{L}(x, m, \alpha)-\langle\alpha, p\rangle\} .
$$

It can be shown that $\mathcal{A}$ and $\mathcal{B}$ are indeed in duality, i.e. (with suitable spaces for $u, m$ and $w)$

$$
(\mathbf{A})=\sup _{u} \mathcal{A}(u)=\inf _{(m, w)} \mathcal{B}(m, w)=(\mathbf{B}) .
$$

This property relies on Fenchel-Rockafellar duality theorem 107 and the following observation:

$$
(\mathbf{A})=-\inf _{u}\{\mathcal{F}(u)+\mathcal{G}(\Lambda(u))\}, \quad(\mathbf{B})=\min _{(m, w)}\left\{\mathcal{F}^{*}\left(\Lambda^{*}(m, w)\right)+\mathcal{G}^{*}(-m,-w)\right\}
$$

where $\Lambda(u)=\left(\frac{\partial u}{\partial t}+\nu \Delta u, \nabla u\right)$,

$$
\begin{aligned}
& \mathcal{F}(u)=\chi_{T}(u)-\int_{\mathbb{T}^{d}} m_{0}(x) u(0, x) d x, \quad \chi_{T}(u)= \begin{cases}0, & \text { if }\left.u\right|_{t=T}=g \\
+\infty, & \text { otherwise }\end{cases} \\
& \mathcal{G}\left(\varphi_{1}, \varphi_{2}\right)=-\inf _{m \geq 0} \int_{0}^{T} \int_{\mathbb{T}^{d}} m(t, x)\left(\varphi_{1}(t, x)-\mathfrak{H}\left(x, m(t, x), \varphi_{2}(t, x)\right)\right) d x d t .
\end{aligned}
$$

$\mathcal{F}^{*}, \mathcal{G}^{*}$ are the convex conjugates of $\mathcal{F}, \mathcal{G}$, and $\Lambda^{*}$ is the adjoint operator of $\Lambda$.

\subsection{Discrete version of the PDE driven optimal control problem.}

In order to implement optimization methods, we need to discretize the problem introduced above in $\S 5.2$. To alleviate notation, we consider the one dimensional case, i.e., $d=1$. We have in mind a MFC problem in the setting of Example 3.1. in which case $\tilde{\ell}(x, p)$ corresponds to $H_{0}^{*}(x,-p)$. For simplicity, we assume that for every $x, \tilde{\ell}(x, \cdot)$ is a power-like function with exponent greater than 1 .

We introduce the following spaces, respectively for the discrete counterpart of $m, w$, and $u$ :

$$
\mathcal{M}=\mathbb{R}^{\left(N_{T}+1\right) \times N_{h}}, \quad \mathcal{W}=\left(\mathbb{R}^{2}\right)^{N_{T} \times N_{h}}, \quad \mathcal{U}=\mathbb{R}^{N_{T} \times N_{h}} .
$$

Note that at each space-time grid point, we will have two variables for the value of $w$, which will be useful to define an upwind scheme.

We denote by $\tilde{H}_{0}$ a discrete Hamiltonian satisfying the properties $(\tilde{\mathrm{H}} \mathbf{1})(\tilde{\mathrm{H}} 4)$ Let $\tilde{H}_{0}^{*}: \mathbb{T}^{d} \times\left(\mathbb{R}^{2}\right)^{d} \rightarrow \mathbb{R} \cup\{+\infty\}$ be its convex conjugate w.r.t. the $p$ variable:

$$
\tilde{H}_{0}^{*}:(x, \gamma) \mapsto \tilde{H}_{0}^{*}(x, \gamma)=\max _{p \in \mathbb{R}^{2}}\left\{\langle\gamma, p\rangle-\tilde{H}_{0}(x, p)\right\} .
$$


EXAMPLE 5.1. In the setting of Example $3.3, \tilde{H}_{0}\left(x, p_{1}, p_{2}\right)=\frac{1}{2}\left|P_{K}\left(p_{1}, p_{2}\right)\right|^{2}$ with $K=\mathbb{R}_{-} \times \mathbb{R}_{+}$, so

$$
\tilde{H}_{0}^{*}\left(x, \gamma_{1}, \gamma_{2}\right)= \begin{cases}\frac{1}{2}\left(\left|\gamma_{1}\right|^{2}+\left|\gamma_{2}\right|^{2}\right), & \text { if }\left(\gamma_{1}, \gamma_{2}\right) \in K, \\ +\infty, & \text { otherwise. }\end{cases}
$$

A discrete counterpart $\tilde{\mathcal{B}}: \mathcal{M} \times \mathcal{W} \rightarrow \mathbb{R}$ to the functional $\mathcal{B}$ introduced in $(5.3)$ can be defined as

$$
\tilde{\mathcal{B}}:(M, W) \mapsto \sum_{n=1}^{N_{T}} \sum_{i=0}^{N_{h}-1}\left[\tilde{\mathfrak{L}}\left(x_{i}, M_{i}^{n}, W_{i}^{n-1}\right)+\mathfrak{F}\left(x_{i}, M_{i}^{n}\right)\right]+\frac{1}{\Delta t} \sum_{i=0}^{N_{h}-1} \mathfrak{G}\left(x_{i}, M_{i}^{N_{T}}\right),
$$

where $\tilde{\mathfrak{L}}: \mathbb{T} \times \mathbb{R} \times \mathbb{R}^{2} \rightarrow \mathbb{R} \cup\{+\infty\}$ is a discrete version of $\mathfrak{L}$ defined as:

$$
\tilde{\mathfrak{L}}:(x, m, w) \mapsto \tilde{\mathfrak{L}}(x, m, w)= \begin{cases}m \tilde{H}_{0}^{*}\left(x,-\frac{w}{m}\right), & \text { if } m>0 \text { and } w \in K, \\ 0, & \text { if } m=0, \text { and } w=0, \\ +\infty, & \text { otherwise. }\end{cases}
$$

Furthermore, a discrete version of the linear constraint (5.4) can be written as

$$
\Sigma(M, W)=\left(0_{\mathcal{U}}, \bar{M}^{0}\right)
$$

where $0_{\mathcal{U}} \in \mathcal{U}$ is the vector with 0 on all coordinates, $\bar{M}^{0}=\left(\bar{m}_{0}\left(x_{0}\right), \ldots, \bar{m}_{0}\left(x_{N_{h}-1}\right)\right) \in$ $\mathbb{R}^{N_{h}}$, see $(3.10)$ for the definition of $\bar{m}_{0}$, and

$$
\Sigma: \mathcal{M} \times \mathcal{W} \rightarrow \mathcal{U} \times \mathbb{R}^{N_{h}}, \quad(M, W) \mapsto \Sigma(M, W)=\left(\Lambda(M, W), M^{0}\right),
$$

with

$$
\Lambda(M, W)=A M+B W,
$$

with $A$ and $B$ being discrete versions of respectively the heat operator and the divergence operator, defined as follows:

$A: \mathcal{M} \rightarrow \mathcal{U}, \quad(A M)_{i}^{n}=\frac{M_{i}^{n+1}-M_{i}^{n}}{\Delta t}-\nu\left(\Delta_{h} M^{n+1}\right)_{i}, \quad 0 \leq n<N_{T}, 0 \leq i<N_{h}$, and

$B: \mathcal{W} \rightarrow \mathcal{U}, \quad(B W)_{i}^{n}=\frac{W_{i+1,2}^{n}-W_{i, 2}^{n}}{h}+\frac{W_{i, 1}^{n}-W_{i-1,1}^{n}}{h} \quad 0 \leq n \leq N_{T}, 0 \leq i<N_{h}$.

The discrete counterpart of the (primal) variational problem $(5.3)-(5.4)$ is therefore

$$
\inf _{(M, W) \in \mathcal{M} \times \mathcal{W}} \tilde{\mathcal{B}}(M, W), \quad \text { subject to } \Sigma(M, W)=\left(0_{\mathcal{U}}, \bar{M}^{0}\right) .
$$

It can be shown that this problem admits a unique solution, and this solution satisfies $M_{i}^{n}>0$ for all $i \in\left\{0, \ldots, N_{h}\right\}$ and all $n>0$; see e.g. [33, Theorem 3.1] for more details on a special case (see also [1, Section 6] and [34, Theorem 2.1] for similar results respectively in the context of the planning problem and in the context of an ergodic MFG).

Analogously to the continuous setting, the discrete problem admits a dual formulation. Furthermore, a key feature of the discretization we chose is that the optimality condition of the discrete problem coincides with the finite-difference scheme presented in Section 3.2, adapted to the current setting. We refer to 10 , Section 3] and the references therein for more details. 
5.4. ADMM and Primal-dual method. In order to draw a connection with numerical methods for optimization problems, let us reformulate the problem from a more abstract perspective. We consider the following primal problem:

$$
\inf _{\xi \in \mathbb{R}^{\mathrm{d}}} \varphi(\xi) \text {, subject to } \Xi \xi=0,
$$

where $\varphi: \mathbb{R}^{\mathrm{d}} \rightarrow(-\infty,+\infty]$ is a lower semi-continuous convex proper function, $\Xi: \mathbb{R}^{\mathrm{d}} \rightarrow \mathbb{R}^{\mathrm{d}^{\prime}}$ is a linear operator, and $0 \in \mathbb{R}^{\mathrm{d}^{\prime}}$. In the setting of the variational mean-field problem described above, problem (5.14) can cover (5.13) with:

$$
\mathrm{d}=2\left(N_{T}+1\right) N_{T}\left(N_{h}\right)^{2}, \quad \xi=(M, W), \quad \varphi=\tilde{\mathcal{B}}, \quad \Xi=\Sigma .
$$

Equivalently, the above primal problem can be written as follows:

$$
\inf _{\xi \in \mathbb{R}^{\mathrm{d}}} \varphi(\xi)+\psi(\widetilde{\Xi} \xi)
$$

where, for $y \in \mathbb{R}^{\mathrm{d}^{\prime}}$,

or

$$
\psi(y)=\iota_{\{0\}}(y)=\left\{\begin{array}{ll}
0, & \text { if } y=0, \\
+\infty, & \text { otherwise, }
\end{array} \quad \text { and } \widetilde{\Xi}=\Xi,\right.
$$

$$
\psi(y)=\iota_{\{0\}}(\Xi y)=\left\{\begin{array}{ll}
0, & \text { if } \Xi y=0, \\
+\infty, & \text { otherwise, }
\end{array} \quad \text { and } \widetilde{\Xi}=\mathrm{id} .\right.
$$

Problems of the form 5.15 have been extensively studied and various numerical methods have been introduced. Here, we will focus on two of them: the Alternating Direction Method of Multipliers for the Augmented Lagrangian of the dual problem, and a primal-dual method proposed by Chambolle and Pock [60. Some of the main advantages of these methods are that proofs of convergence are readily available, and they can be applied to first order problems (i.e., when there is no diffusion and $\nu=0$ ).

Augmented Lagrangian and ADMM. We first note that 5.15) admits as a dual formulation the following problem:

$$
\inf _{\zeta \in \mathbb{R}^{d^{\prime}}} \varphi^{*}\left(-\widetilde{\Xi}^{*} \zeta\right)+\psi^{*}(\zeta)
$$

where $\varphi^{*}$ and $\psi^{*}$ are the convex conjugates of $\varphi$ and $\psi$ respectively and $\widetilde{\Xi}^{*}$ is the adjoint operator of $\widetilde{\Xi}$, i.e., $\langle\widetilde{\Xi} \xi, \zeta\rangle=\left\langle\xi, \widetilde{\Xi}^{*} \zeta\right\rangle$. In the setting of $\S 5.2$ and $\S 5.3 \zeta$ plays the role of a vector $U \in \mathcal{U}$ approximating the function $u$ appearing in the dual problem (5.5).

For numerical purposes, it is useful to exploit the additive structure of the objective function and introduce an extra variable to rewrite the problem as:

$$
\inf _{\zeta, \nu \in \mathbb{R}^{d^{\prime}}} \varphi^{*}(\nu)+\psi^{*}(\zeta) \text {, subject to } \nu=-\widetilde{\Xi}^{*} \zeta \text {. }
$$

The Lagrangian associated to this constrained optimization problem is defined, introducing a Lagrange multiplier $\lambda$, as:

$$
\mathcal{L}(\zeta, \nu, \lambda)=\varphi^{*}(\nu)+\psi^{*}(\zeta)+\left\langle\lambda, \nu+\widetilde{\Xi}^{*} \zeta\right\rangle .
$$

Solving the dual problem 5.16 thus amounts to finding a saddle point of $\mathcal{L}$, i.e., $\left(\zeta^{*}, \nu^{*}, \lambda^{*}\right)$ achieving

$$
\mathcal{L}\left(\zeta^{*}, \nu^{*}, \lambda^{*}\right)=\sup _{\lambda} \inf _{\zeta, \nu} \mathcal{L}(\zeta, \nu, \lambda) .
$$


This suggests to use a steepest descent method in order to approximate the maximizer of $\lambda \mapsto \inf _{\zeta, \nu} \mathcal{L}(\zeta, \nu, \lambda)$, where the inf can be split into two separate optimization sub-problems. For more details on this method, see the algorithm ALG1 in 69, Chapter 3]. A variation of this approach consists in adding an extra penalty term to obtain the so-called Augmented Lagrangian defined, for $r>0$, as:

$$
\mathcal{L}_{r}(\zeta, \nu, \lambda)=\varphi^{*}(\nu)+\psi^{*}(\zeta)+\left\langle\lambda, \nu+\widetilde{\Xi}^{*} \zeta\right\rangle+\frac{r}{2}\left\|\nu+\widetilde{\Xi}^{*} \zeta\right\|_{2}^{2} .
$$

This new function can be interpreted as the Lagrangian of a modified version of the dual problem (5.16) in which the penalty term $\frac{r}{2}\left\|\nu+\widetilde{\Xi}^{*} \zeta\right\|_{2}^{2}$ is added to the objective. The primal problem corresponding to this penalized dual problem can be interpreted as a regularized version of (5.14). Note that $\mathcal{L}$ and $\mathcal{L}_{r}$ have the same saddle point, for any $r>0$.

The principle of the Alternating Direction Method of Multipliers applied to this Augmented Lagrangian $\mathcal{L}_{r}$ is to iteratively update in turn $\zeta, \nu$ and $\lambda$, as summarized in Algorithm 4 See the algorithm ALG2 in [69, Chapter 3] for more details. Here prox denotes the proximal operator defined, for a lower semicontinuous convex proper function $\varphi: \mathbb{R}^{\mathrm{d}} \rightarrow(-\infty,+\infty]$ as:

$$
\operatorname{prox}_{\varphi}(y)=\underset{y^{\prime}}{\arg \min }\left\{\varphi\left(y^{\prime}\right)+\frac{1}{2}\left\|y^{\prime}-y\right\|^{2}\right\} .
$$

It generalizes the notion of orthogonal projection in the sense that if $K \subseteq \mathbb{R}^{\mathrm{d}}$ is a non-empty, closed, convex set and $\iota_{K}: \mathbb{R}^{\mathrm{d}} \rightarrow\{0,+\infty\}$ denotes its characteristic function, then $\operatorname{prox}_{\iota_{K}}$ is the orthogonal projection on $K$. In Algorithm 4 , the first two steps (optimization with respect to $\zeta$ and proximal step for $\nu$ ) are the most costly in terms of computation.

The convergence of this method can be ensured under suitable conditions, see [67, Theorem 8] for more details.

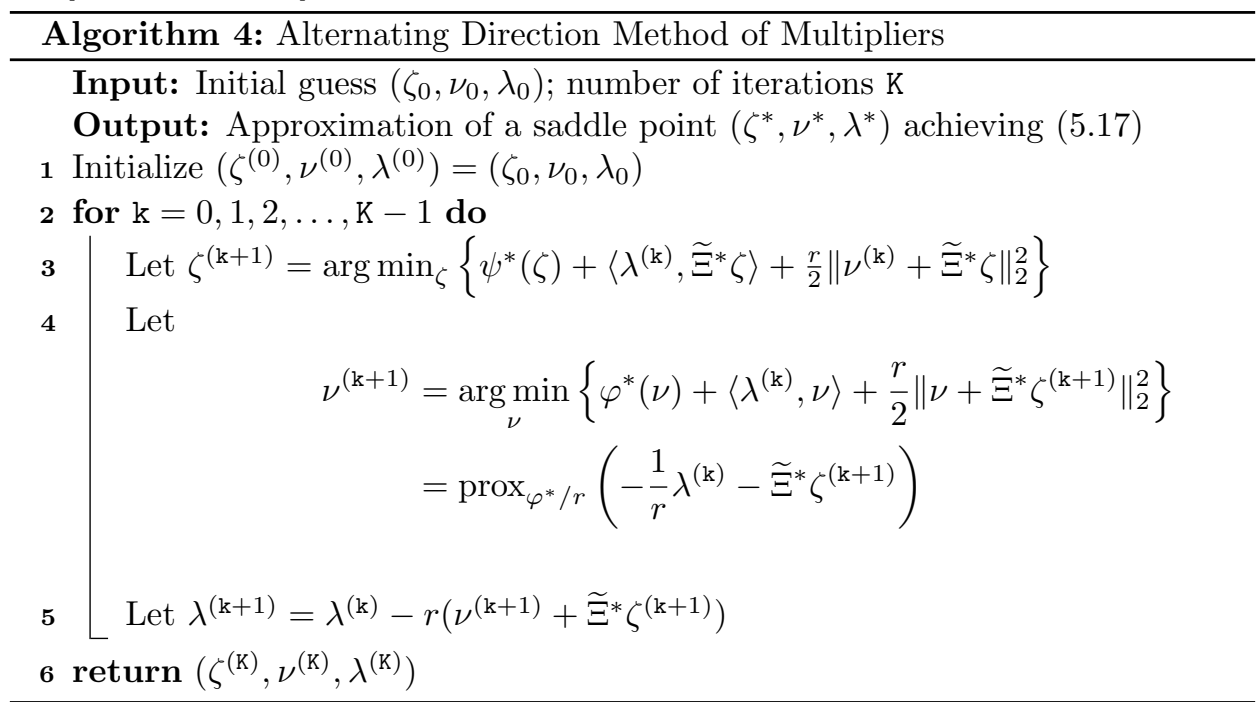

The ADMM was made popular by Benamou and Brenier for optimal transport, see [27, and first used in the context of MFGs by Benamou and Carlier in [28; see also 20] for an application to second order MFG using multilevel preconditioners and $[9$ for an application to mean field type control. 
When applied to the dual of the variational problem (5.13), in the first step, the first order optimality condition for the minimization yields that $U^{(\mathrm{k}+1)}$ is the solution to a finite difference equation which in the general case $\nu>0$ corresponds to a PDE with a fourth order elliptic operator. Then a preconditioner is needed, see e.g. [12, 33, except if $\nu=0$, in which case a direct solver can be used. In the second step, the minimization problem can be done separately at each point of the grid, which allows parallelization.

Chambolle and Pock's primal-dual algorithm. We now turn our attention to an algorithm proposed in 60 which relies on both the primal and the dual problems (5.15)-(5.16). It is based on the Lagrangian:

$$
\mathcal{L}_{P D}(\xi, \zeta)=\varphi(\xi)-\psi^{*}(\zeta)+\langle\zeta, \widetilde{\Xi} \xi\rangle,
$$

for which the optimality conditions in each variable are:

$$
\begin{aligned}
\begin{cases}\widetilde{\Xi}^{*} \hat{\zeta} \in-\partial \varphi(\hat{\xi}) \\
\widetilde{\Xi} \hat{\xi} \in \partial \psi^{*}(\hat{\zeta})\end{cases} & \Leftrightarrow\left\{\begin{array}{l}
\hat{\xi}-\tau \widetilde{\Xi}^{*} \hat{\zeta} \in \hat{\xi}+\tau \partial \varphi(\hat{\xi}) \\
\hat{\zeta}+\gamma \widetilde{\Xi} \hat{\xi} \in \hat{\zeta}+\gamma \partial \psi^{*}(\hat{\zeta})
\end{array}\right. \\
& \Leftrightarrow\left\{\begin{array}{l}
\hat{\zeta} \in \arg \min _{\zeta}\left\{\psi^{*}(\zeta)+\frac{1}{2 \gamma}\|\zeta-(\hat{\zeta}+\gamma \widetilde{\Xi} \hat{\xi})\|^{2}\right\} \\
\hat{\xi} \in \arg \min _{\xi}\left\{\varphi(\xi)+\frac{1}{2 \tau}\left\|\xi-\left(\hat{\xi}-\tau \widetilde{\Xi} \widetilde{\zeta}^{*}\right)\right\|^{2}\right.
\end{array}\right\} \\
& \Leftrightarrow \begin{cases}\hat{\zeta} \in \operatorname{prox}_{\gamma \psi^{*}}(\hat{\zeta}+\gamma \widetilde{\Xi} \hat{\xi}) \\
\hat{\xi} \in \operatorname{prox}_{\tau \varphi}\left(\hat{\xi}-\tau \widetilde{\Xi} \widetilde{\zeta}^{*}\right) .\end{cases}
\end{aligned}
$$

The method proposed by Chambolle and Pock is basically to iterate over the two proximal steps, with an additional extrapolation step, as summarized in Algorithm 5 . The method has been proved to converged when $\gamma \tau<1$, see [60.

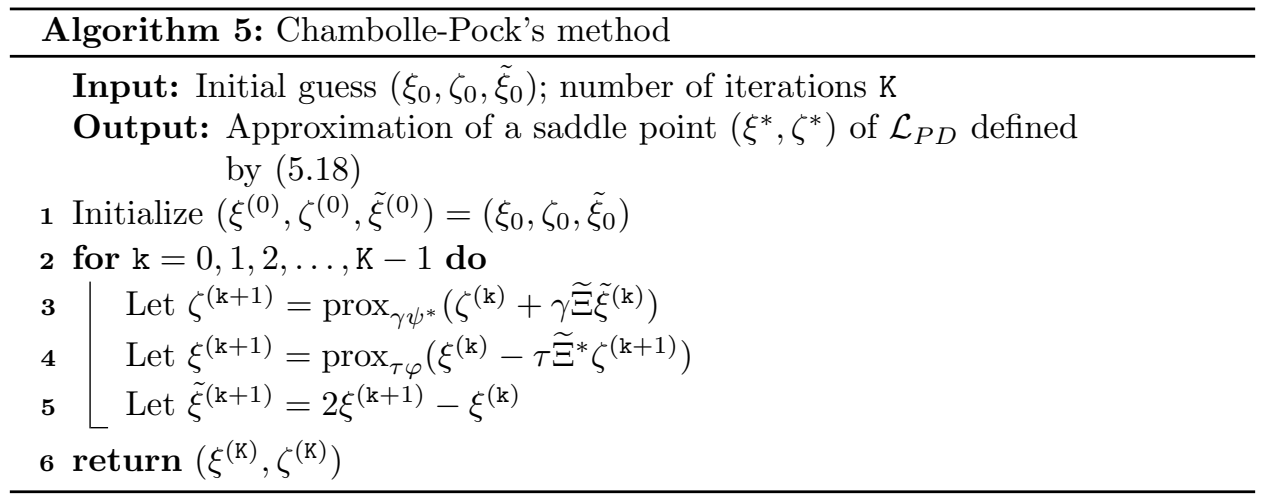

The application of this method to stationary MFGs was first investigated by Briceño-Arias, Kalise and Silva in $\mathbf{3 4}$; see also $\mathbf{3 3}$ for an extension to the dynamic setting. When applied to the problem (5.13), the first step is similar to the first step in the ADMM method described above and amounts to solving a linear fourth order PDE. The second step is easier thanks to the choice of $\mathcal{B}$ and the form of its prox.

5.5. Numerical illustration: crowd motion without diffusion. We present an example, borrowed from [9], of a mean field model for crowd motion with congestion. The interactions are local and, in contrast with the example of $\S 4.4$, there is no diffusion, i.e., $\nu=0$. Due to the fact that the running cost is not separable 
in $\alpha$ and $m$, the MFG does not have a variational structure. We focus here on the MFC problem.

On top of the congestion effects (meaning that moving quickly in a dense region is expensive), we incorporate aversion effects (i.e., being in a crowded region is uncomfortable). The latter aspect is modeled by a function $\ell: \mathcal{Q} \times \mathbb{R} \rightarrow \mathbb{R},(x, m) \mapsto$ $\ell(x, m)$ which is increasing with respect to $m$. The domain is $\mathcal{Q}=[0,1]^{2} \backslash[0.4,0.6]^{2}$, which is a square with obstacle at the center. The Lagrangian (corresponding to the running cost) is:

$$
L(x, m, \alpha)=(b-1)^{b^{*}} m^{\frac{a}{b-1}}|\alpha|^{b^{*}}+\ell(x, m), \quad 1<b \leq 2,0 \leq a<1,
$$

where $b^{*}=b /(b-1)$ is the conjugate exponent of $b$. We define the Hamiltonian by duality (such that on $\partial \Omega$ the vector speed is towards the interior):

$$
H(x, m, p)= \begin{cases}\inf _{\alpha \in \mathbb{R}^{2}}\{\alpha \cdot p+L(x, m, \alpha)\}=-m^{-a}|p|^{b}+\ell(x, m), & \text { if } x \in \Omega, \\ \inf _{\alpha \in \mathbb{R}^{2}: \alpha \cdot \mathbf{n} \leq 0}\{\alpha \cdot p+L(x, m, \alpha)\}, & \text { if } x \in \partial \Omega,\end{cases}
$$

where $\mathbf{n}$ denotes the outward normal.

As shown in Figure 13 we take $m_{0}$ uniform in a corner and the terminal cost $g$ minimal at the opposite corner. For the numerical experiments reported here, we took $a=0.01, b=2, \ell(x, m)=0.01 \mathrm{~m}$. The evolution of the density is displayed in Figure 14. We see that it avoids the obstacle and ends up in the opposite corner. Since high velocity is prohibitive (and there is no terminal cost penalizing high density), most of the mass concentrates near the arrival point. Due to the absence of diffusion, at every time step the density remains zero on a large part of the domain. Here, we used the ADMM on the Augmented Lagrangian for the discrete problem as discussed above in this section.
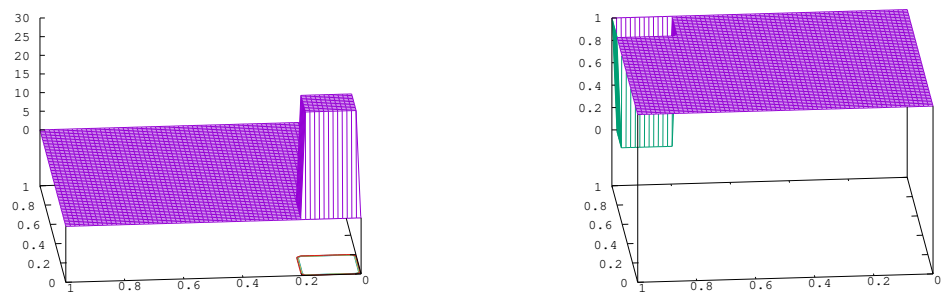

FiguRE 13. Left: Initial distribution $m_{0}$. Right: terminal cost $g$. 



$t=T / 4$

$t=T / 2$
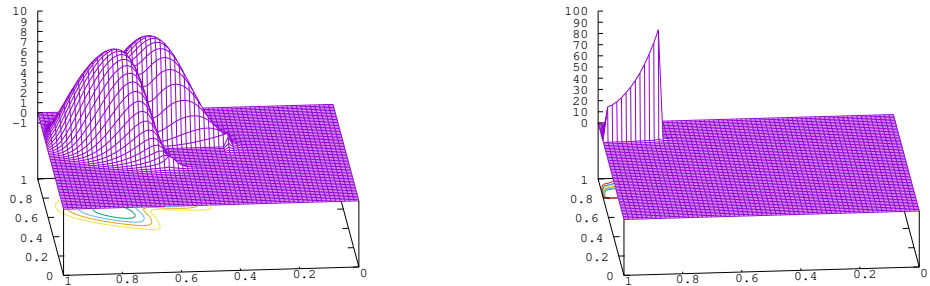

$t=3 T / 4$

$$
t=T
$$

Figure 14. Evolution of the density. 


\section{A Method based on monotone operators}

Almulla, Ferreira and Gomes proposed in [17. two numerical methods for mean field games in infinite horizon, in which the solution is stationary. These methods are further studied in [77, [78. The first method can be applied to variational MFG (or more generally to MFC) for which the PDE system corresponds to the Euler-Lagrange conditions arising in the minimization of some energy functional. The idea is then to follow the gradient-flow generated by this energy until reaching a zero of the gradient.

The second method can be applied more broadly to infinite horizon MFGs, provided a monotonicity condition is satisfied. The main idea is that, under some assumptions, the solution to the system of PDEs can be recast as a zero of a monotone operator. The strategy is then to follow the flow generated by this operator until finding a zero.

6.1. A Monotonic flow. We focus here on the second method. Let us consider the case of Example 3.1 when, in addition, the dependence on the distribution is local and more specifically $f_{0}(x, m)=\log (m(x))$. The ergodic MFG (see e.g. 93 ) leads to the following system of PDEs:

$$
\begin{cases}0=\lambda-\nu \Delta u(x)+H_{0}(x, \nabla u(x))-\log (m(x)), & \text { in } \mathbb{T}^{d} \\ 0=-\nu \Delta m(x)-\operatorname{div}\left(m \partial_{p} H_{0}(x, \nabla u(x))\right), & \text { in } \mathbb{T}^{d} \\ \int_{\mathbb{T}^{d}} m=1, \quad \int_{\mathbb{T}^{d}} u=0, \quad m>0 \text { in } \mathbb{T}^{d}, & \end{cases}
$$

where the unknowns are the functions $u$ and $m$, and the ergodic constant $\lambda$. Let $A$ be the operator defined on the domain

$$
\mathcal{D}=\left\{(u, m) \in W^{2,2}\left(\mathbb{T}^{d}\right) \times W^{1,2}\left(\mathbb{T}^{d}\right): \inf _{\mathbb{T}^{d}} m>0\right\} \subseteq L^{2}\left(\mathbb{T}^{d}\right) \times L^{2}\left(\mathbb{T}^{d}\right)
$$

by

$$
A\left(\begin{array}{c}
u \\
m
\end{array}\right)=\left(\begin{array}{c}
-\nu \Delta m-\operatorname{div}\left(m(\cdot) \partial_{p} H_{0}(\cdot, \nabla u(\cdot))\right) \\
\nu \Delta u-H_{0}(\cdot, \nabla u)+\log (m)
\end{array}\right) .
$$

It can be checked that this operator, seen as an operator from $L^{2}\left(\mathbb{T}^{d}\right) \times L^{2}\left(\mathbb{T}^{d}\right)$ to $L^{2}\left(\mathbb{T}^{d}\right) \times L^{2}\left(\mathbb{T}^{d}\right)$, is monotone on its domain, namely,

$$
\left\langle A(u, m)-A\left(u^{\prime}, m^{\prime}\right),(u, m)-\left(u^{\prime}, m^{\prime}\right)\right\rangle \geq 0, \quad(u, m),\left(u^{\prime}, m^{\prime}\right) \in \mathcal{D} .
$$

Then, we can consider the following flow $\left(u_{\tau}, m_{\tau}\right)_{\tau \geq 0}$, where $\left(u_{\tau}, m_{\tau}\right) \in L^{2}([0, T] \times$ $\left.\mathbb{T}^{d}\right) \times L^{2}\left([0, T] \times \mathbb{T}^{d}\right)$ for all $\tau \geq 0$ :

$$
\frac{d}{d \tau}\left(\begin{array}{c}
u_{\tau} \\
m_{\tau}
\end{array}\right)=-A\left(\begin{array}{c}
u_{\tau} \\
m_{\tau}
\end{array}\right)-\left(\begin{array}{c}
0 \\
\lambda_{\tau}
\end{array}\right)
$$

with $\lambda_{\tau}$ such that $\int_{\mathbb{T} d} m_{\tau}=1$. We stress that here $\tau$ denotes the time in the evolution of the flow (and recall that we focus on a stationary MFG here). Under suitable conditions, if the initial point $\left(u_{0}, m_{0}\right)$ is in $\mathcal{D}$, the flow remains in $\mathcal{D}$ until it reaches a point where the right-hand side is 0 , i.e., the solution to the ergodic MFG PDE system.

This evolution can be approximated using the finite difference discretization introduced in $\S 3.2$. More precisely, if the state space is of dimension $d=1$, we 
consider the following system for $n=0,1, \ldots$ :

$$
\left(\begin{array}{c}
\left(D_{t} U\right)^{n} \\
\left(D_{t} M\right)^{n}
\end{array}\right)=-\left(\begin{array}{c}
-\nu\left(\Delta_{h} M^{n+1}\right)_{i}+\mathcal{T}_{i}\left(U^{n+1}, M^{n+1}\right) \\
\lambda^{n+1}+\nu\left(\Delta_{h} U^{n+1}\right)_{i}-\tilde{H}_{0}\left(x_{i},\left[\nabla_{h} U^{n+1}\right]_{i}\right)+\log \left(M_{i}^{n+1}\right)
\end{array}\right)
$$

where $\lambda^{n+1}$ is such that $\sum_{i} M_{i}^{n+1}=1$. For any $n \geq 0$, assuming that $\sum_{i} M_{i}^{n}=1$ and summing over $i$ the second half of the above system of equations yields

$$
\begin{aligned}
& \lambda^{n+1} \text { is such that } \sum_{i} M^{n+1}=1 \\
\Leftrightarrow & 0=N_{h} \lambda^{n+1}+\sum_{i}\left[\nu\left(\Delta_{h} U^{n+1}\right)_{i}-\tilde{H}_{0}\left(x_{i},\left[\nabla_{h} U^{n+1}\right]_{i}\right)+\log \left(M_{i}^{n+1}\right)\right] \\
\Leftrightarrow & \lambda^{n+1}=\psi\left(U^{n+1}, M^{n+1}\right),
\end{aligned}
$$

where $\psi(U, M)=-h \sum_{i}\left[\nu\left(\Delta_{h} U\right)_{i}-\tilde{H}_{0}\left(x_{i},\left[\nabla_{h} U\right]_{i}\right)+\log \left(M_{i}\right)\right]$, for $U, M \in \mathbb{R}^{N_{h}+1}$.

This leads directly to an iterative procedure, by starting from an initial guess $\left(U^{0}, M^{0}\right)$ and repeating the following step for increasing values of $n=0,1, \ldots$ :

$$
\left(\begin{array}{c}
\left(D_{t} U\right)^{n} \\
\left(D_{t} M\right)^{n}
\end{array}\right)=-\left(\begin{array}{c}
-\nu\left(\Delta_{h} M^{n+1}\right)_{i}+\mathcal{T}_{i}\left(U^{n+1}, M^{n+1}\right) \\
\psi\left(U^{n+1}, M^{n+1}\right)+\nu\left(\Delta_{h} U^{n+1}\right)_{i}-\tilde{H}_{0}\left(x_{i},\left[\nabla_{h} U^{n+1}\right]_{i}\right)+\log \left(M_{i}^{n+1}\right)
\end{array}\right),
$$

At each step, this computation involves solving a non-linear system to obtain $\left(U^{n+1}, M^{n+1}\right)$, which can be done with Newton method for example. In order to tackle the fact that $M_{i}$ must remain positive for every $i$ (in order for the log to make sense), one can for instance use a damped version of Newton iterations.

6.2. Numerical illustration. We illustrate this method on two examples. To the best of our knowledge, the convergence has been proved only for first order (i.e., $\nu=0$ ) stationary problems with a logarithmic coupling, see [17. For other coupling costs, a projection step might be needed in order to ensure non-negativity of the distribution.

Test case 1 (first order). The following example is borrowed from [17. We consider the case where $d=1, \nu=0$ and $H_{0}$ is of the form

$$
H_{0}(x, p)=\frac{1}{2}|p|^{2}+b(x) p+V(x)
$$

with $b(x)=2 c \pi \cos (2 \pi x)$ where $c \in[0,1]$, and

$$
V(x)=\sin (2 \pi x) .
$$

In this case, the system 6.1 admits a unique solution, given explicitly by

$$
u^{*}(x)=c \sin (2 \pi x), \quad m^{*}(x)=\frac{e^{V(x)-\frac{b(x)^{2}}{2}}}{\int_{\mathbb{T}} e^{V(y)-\frac{b(y)^{2}}{2}} d y}, \quad \lambda=\int_{\mathbb{T}} e^{V(y)-\frac{b(y)^{2}}{2}} d y .
$$

We then consider the following discrete Hamiltonian (which is a modification of the one discussed in Example 3.3 to incorporate the effect of the extra terms with $b$ and $V)$ :

$$
\tilde{H}_{0}\left(x, p_{1}, p_{2}\right)=\tilde{H}_{0}^{(1)}\left(p_{1}, p_{2}\right)+\tilde{H}_{0}^{(2)}\left(x, p_{1}, p_{2}\right)+V(x),
$$

with

$$
\tilde{H}_{0}^{(1)}\left(p_{1}, p_{2}\right)=\frac{1}{2}\left|P_{K}\left(p_{1}, p_{2}\right)\right|^{2}, \quad \tilde{H}_{0}^{(2)}\left(x, p_{1}, p_{2}\right)= \begin{cases}b(x) p_{1}, & \text { if } b(x) \leq 0, \\ b(x) p_{2}, & \text { otherwise }\end{cases}
$$



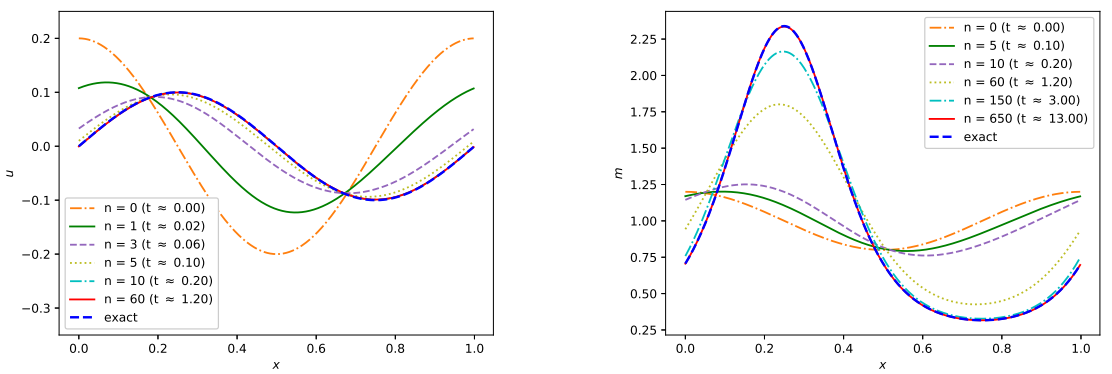

Figure 15. Test case 1: Value function $U^{n}$ (left) and density $M^{n}$ (right) for several values of $n$, following the evolution 6.2.
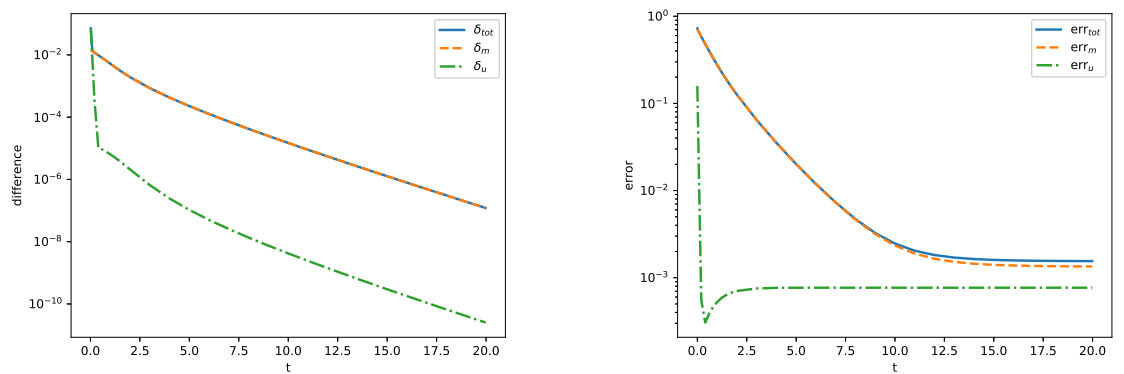

Figure 16. Test case 1: Difference between two iterations of the monotonic flow $(6.2)$ (left) and error with respect to the exact solution (right), computed as indicated in the text, see 6.3)- 6.4.

where $P_{K}$ denotes the projection on $K=\mathbb{R}_{-} \times \mathbb{R}_{+}$. Figure 15 displays $U^{n}$ and $M^{n}$ for several values of $n$ (note that the values of $n$ are not the same in each figure). Figure 16 displays the $L^{2}$ difference between two iterations and the $L^{2}$ error with respect to the exact solution, defined respectively by:

$\delta_{u}^{n}=\sqrt{h \sum_{i}\left|U_{i}^{n}-U_{i}^{n-1}\right|^{2}}, \quad \delta_{m}^{n}=\sqrt{h \sum_{i}\left|M_{i}^{n}-M_{i}^{n-1}\right|^{2}}, \quad \delta_{t o t}^{n}=\sqrt{\left|\delta_{u}^{n}\right|^{2}+\left|\delta_{m}^{n}\right|^{2}}$,

and

$(6.4)$

$\operatorname{err}_{u}^{n}=\sqrt{h \sum_{i}\left|U_{i}^{n}-U_{i}^{*}\right|^{2}}, \quad \operatorname{err}_{m}^{n}=\sqrt{h \sum_{i}\left|M_{i}^{n}-M_{i}^{*}\right|^{2}}, \quad \operatorname{err}_{t o t}^{n}=\sqrt{\left|\operatorname{err}_{u}^{n}\right|^{2}+\left|\operatorname{err}_{m}^{n}\right|^{2}}$,

where

$$
U_{i}^{*}=u^{*}\left(x_{i}\right), \quad M_{i}^{*}=m^{*}\left(x_{i}\right),
$$

are given by the explicit solution. In the numerical computations to obtain these figures, we used $c=0.1, N_{h}=500, T=20, N_{T}=1000$.

Test case 2 (second order). We now consider an example of secondorder MFG (i.e., with $\nu>0$ ) admitting an explicit solution. Although, to the 

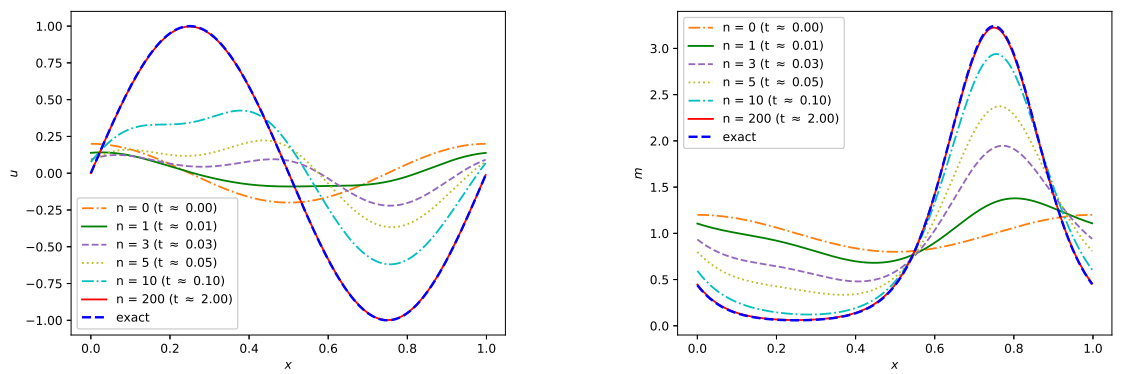

Figure 17. Test case 2: Value function $U^{n}$ (left) and density $M^{n}$ (right) for several values of $n$, following the evolution 6.2.
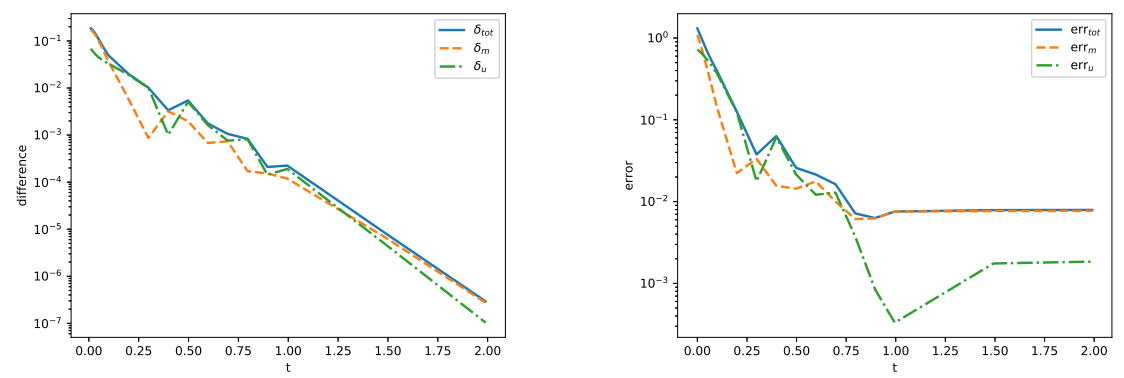

Figure 18. Test case 2: Difference between two iterations of the monotonic flow (6.2) (left) and error with respect to the exact solution (right), computed as indicated in the text, see 6.3)-6.4.

best of our knowledge, the scheme has not been proved to converge in this setting, the numerical results seems to indicate that convergence holds. Let $b=0, \nu=$ $0.5, V(x)=2 \pi^{2}\left(-\kappa \sin (2 \pi x)-\kappa^{2} \cos ^{2}(2 \pi x)\right)-2 \kappa \sin (2 \pi x)$, where $\kappa$ is a constant. Then the explicit solution is given by

$$
u(x)=\kappa \sin (2 \pi x), \quad m(x)=\kappa \frac{e^{2 u(x)}}{\int e^{2 u}} .
$$

We show the results for $\kappa=1.0$. See Figures 17 and 18 


\section{Methods based on approximation by neural networks}

In this section, we present methods introduced recently which are based on tools borrowed from machine learning. To wit, we will use (artificial) neural networks to approximate functions of interest, and stochastic gradient descent to optimize over the weights of these neural networks. The first method we present is for MFC (or variational MFG) and directly based on the stochastic formulation. The second method tackles the master equation for finite-state mean-field problems ${ }^{2}$

7.1. Direct optimization for MFC. Since MFC is an optimization problem, we present a first method which uses stochastic optimization tools from machine learning and which is directly based on the definition $(1.3)-(1.4)$ of the MFC problem. Starting from this formulation, we make three approximations in order to obtain a new problem that is more amenable to numerical treatment.

Approximation steps. First, we restrict the set of (feedback) controls to be the set of neural networks with a given architecture. We introduce new notation to define this class of controls. We denote by:

$$
\begin{gathered}
\mathbf{L}_{d_{1}, d_{2}}^{\psi}=\left\{\phi: \mathbb{R}^{d_{1}} \rightarrow \mathbb{R}^{d_{2}} \mid \exists(\beta, w) \in \mathbb{R}^{d_{2}} \times \mathbb{R}^{d_{2} \times d_{1}}, \forall i \in\left\{1, \ldots, d_{2}\right\},\right. \\
\left.\phi(x)_{i}=\psi\left(\beta_{i}+\sum_{j=1}^{d_{1}} w_{i, j} x_{j}\right)\right\}
\end{gathered}
$$

the set of layer functions with input dimension $d_{1}$, output dimension $d_{2}$, and activation function $\psi: \mathbb{R} \rightarrow \mathbb{R}$. Typical choices for $\psi$ are $\operatorname{ReLU}$ function $\psi(x)=x^{+}$or the sigmoid function $\psi(x)=1 /\left(1+e^{-x}\right)$. Building on this notation and denoting by $\circ$ the composition of functions, we define:

$$
\begin{gathered}
\mathbf{N}_{d_{0}, \ldots, d_{\ell+1}}^{\psi}=\left\{\varphi: \mathbb{R}^{d_{0}} \rightarrow \mathbb{R}^{d_{\ell+1}} \mid \exists\left(\phi_{i}\right)_{i=0, \ldots, \ell-1} \in \underset{i=0}{\times} \mathbf{L}_{d_{i}, d_{i+1}}^{\psi},\right. \\
\left.\exists \phi_{\ell} \in \mathbf{L}_{d_{\ell}, d_{\ell+1}}^{\mathrm{id}}, \varphi=\phi_{\ell} \circ \phi_{\ell-1} \circ \cdots \circ \phi_{0}\right\}
\end{gathered}
$$

the set of regression neural networks with $\ell$ hidden layers and one output layer, the activation function of the output layer being the identity. The number $\ell$ of hidden layers, the numbers $d_{0}, d_{1}, \cdots, d_{\ell+1}$ of units per layer, and the activation functions (one single function $\psi$ in the present situation), are what is usually called the architecture of the network. Once it is fixed, the actual network function $\varphi \in \mathbf{N}_{d_{0}, \ldots, d_{\ell+1}}^{\psi}$ is determined by the remaining real-valued parameters:

$$
\theta=\left(\beta^{(0)}, w^{(0)}, \beta^{(1)}, w^{(1)}, \ldots \ldots, \beta^{(\ell-1)}, w^{(\ell-1)}, \beta^{(\ell)}, w^{(\ell)}\right)
$$

defining the functions $\phi_{0}, \phi_{1}, \cdots, \phi_{\ell-1}$ and $\phi_{\ell}$ respectively. The set of such parameters is denoted by $\Theta$. For each $\theta \in \Theta$, the function $\varphi$ computed by the network will be denoted by $\alpha_{\theta} \in \mathbf{N}_{d_{0}, \ldots, d_{\ell+1}}^{\psi}$. As it should be clear from the discussion of the previous section, here, we are interested in the case where $d_{0}=d+1$ (since the inputs are time and state) and $d_{\ell+1}=k$ (i.e., the control dimension). The problem becomes to minimize $J^{M F C}$ defined by 1.3 -1.4 over $\alpha \in \mathbf{N}_{d+1, d_{1}, \ldots, d_{\ell}, k}^{\psi}$, or

\footnotetext{
${ }^{2}$ For the sake of brevity, we do not discuss here other neural network based methods which have been developed for instance for the systems of partial differential equations or stochastic differential equations of MFGs. See the references in this section and in the conclusion.
} 
equivalently, to minimize over $\theta \in \Theta$ the function:

$$
\mathbf{J}: \theta \mapsto \mathbb{E}\left[\int_{0}^{T} f\left(X_{t}^{\alpha_{\theta}}, m^{\alpha_{\theta}}(t, \cdot), \alpha_{\theta}\left(t, X_{t}^{\alpha_{\theta}}\right)\right) d t+g\left(X_{T}^{\alpha_{\theta}}, m^{\alpha_{\theta}}(T, \cdot)\right)\right]
$$

where $m^{\alpha_{\theta}}(t, \cdot)$ is the probability density of the law of $X_{t}^{\alpha_{\theta}}$, under the constraint that the process $X^{\alpha_{\theta}}$ solves the SDE (1.4) with control $\alpha_{\theta}$.

Second, we also need to approximate the density. A (computationally) simple option is to replace it by the empirical distribution of a system of $N$ interacting particles. Given a feedback control $\alpha$, we denote by $\left(\underline{X}_{t}^{\alpha}\right)_{t}=\left(X_{t}^{1, \alpha}, \ldots, X_{t}^{N, \alpha}\right)_{t}$ the solution of:

$$
d X_{t}^{i, \alpha}=b\left(X_{t}^{i, \alpha}, m_{t}^{N, \alpha}, \alpha\left(t, X_{t}^{i, \alpha}\right)\right) d t+\sigma d W_{t}^{i}, \quad t \geq 0,
$$

where

$$
m_{t}^{N, \alpha}=\frac{1}{N} \sum_{j=1}^{N} \delta_{X_{t}^{j, \alpha}},
$$

$\left(W^{i}\right)_{i=1, \ldots, N}$ is a family of $N$ independent $d$-dimensional Brownian motions, and the initial positions $\left(X_{0}^{i, \alpha}\right)_{i=1, \ldots, N}$ are i.i.d. with distribution given by the density $m_{0}$. Note that in 7.2 the particles interact only through $m_{t}^{N, \alpha}$ in the drift function. The controls are distributed in the sense that the control used in the dynamics of $X^{i, \alpha}$ is a function of $t$ and $X_{t}^{i, \alpha}$ itself only (and not of the position of the other particles). This choice is motivated by the fact that MFC and $N$-agent control problems with distributed controls are tightly connected, see [53. We thus obtain the following new problem: Minimize over $\theta \in \Theta$ the function

$$
\mathbf{J}^{N}: \theta \mapsto \frac{1}{N} \sum_{i=1}^{N} \mathbb{E}\left[\int_{0}^{T} f\left(X_{t}^{i, \alpha_{\theta}}, m_{t}^{N, \alpha_{\theta}}, \alpha_{\theta}\left(t, X_{t}^{i, \alpha_{\theta}}\right)\right) d t+g\left(X_{T}^{i, \alpha_{\theta}}, m_{T}^{N, \alpha_{\theta}}\right)\right],
$$

under the dynamics 7.2 with control $\alpha_{\theta}$. The average over $i$ is here for the sake of analogy with $N$-player games, but each expectation in the sum has the same value since the agents are identically distributed. We interpret $\alpha_{\theta}\left(t, X_{t}^{i, \alpha_{\theta}}\right)$ as the control used by player $i$ at time $t$. Note that it can be viewed as a feedback control, function of time and player $i$ 's state. Since it is not a function of the empirical distribution $m_{t}^{N, \alpha_{\theta}}$, one may wonder how the solution to this problem could be close to the solution of the corresponding MFC. This is where a mean-field approximation is helpful: player $i$ 's control can implicitly depend on the MFC distribution through its input $t$. Intuitively, the MFC distribution $m^{\alpha_{\theta}}(t, \cdot)$ is a deterministic proxy for the $N$-agent empirical (stochastic) distribution $m_{t}^{N, \alpha_{\theta}}$. This is because the initial distribution is given and the idiosyncratic noises disappear in the limiting distribution evolution.

Last, the time interval is discretized. Let $N_{T}$ be a positive number, let $\Delta t=$ $T / N_{T}$ and $t_{n}=n \Delta t, n=0, \ldots, N_{T}$. We consider the problem: Minimize over $\theta \in \Theta$ the function

$$
\mathbf{J}^{N, \Delta t}: \theta \mapsto \frac{1}{N} \sum_{i=1}^{N} \mathbb{E}\left[\sum_{n=0}^{N_{T}-1} f\left(\check{X}_{t_{n}}^{i, \alpha_{\theta}}, \check{m}_{t_{n}}^{N, \alpha_{\theta}}, \alpha_{\theta}\left(t_{n}, \check{X}_{t_{n}}^{i, \alpha_{\theta}}\right)\right) \Delta t+g\left(\check{X}_{T}^{i, \alpha_{\theta}}, \check{m}_{T}^{N, \alpha_{\theta}}\right)\right]
$$


under the dynamic constraint:

(7.4)

$\check{X}_{t_{n+1}}^{i, \alpha_{\theta}}=\check{X}_{t_{n}}^{i, \alpha_{\theta}}+b\left(\check{X}_{t_{n}}^{i, \alpha_{\theta}}, \check{m}_{t_{n}}^{N, \alpha_{\theta}}, \alpha_{\theta}\left(t, \check{X}_{t_{n}}^{i, \alpha_{\theta}}\right)\right) \Delta t+\sigma \Delta \check{W}_{n}^{i}, \quad n=0, \ldots, N_{T}-1$,

and the initial positions $\left(\check{X}_{0}^{i, \alpha_{\theta}}\right)_{i=1, \ldots, N}$ are i.i.d. with distribution given by the density $m_{0}$, where

$$
\check{m}_{t_{n}}^{N, \alpha_{\theta}}=\frac{1}{N} \sum_{j=1}^{N} \delta_{\check{X}_{t_{n}}^{j, \alpha_{\theta}}}
$$

and the $\left(\Delta \breve{W}_{n}^{i}\right)_{i=1, \ldots, N, n=0, \ldots, N_{T}-1}$ are i.i.d. random variables with Gaussian distribution $\mathcal{N}(0, \Delta t)$.

Under suitable assumptions on the problem and the neural network architecture, the difference between $\inf _{\theta} \mathbf{J}^{N, \Delta t}(\theta)$ and $\inf _{\alpha} J^{M F C(\alpha)}$ goes to 0 as $N_{t}, N$ and the number of parameters in the neural network go to infinity. See [55] for more details.

Optimization procedure. Note that the cost function 7.3 is in general non-convex due to the complicated way in which $\theta$ is involved in the cost (particularly since we consider neural networks). Moreover, $\theta$ is typically in (finite but) high dimension. In order to compute an (approximate) optimizer $\theta^{*}$, it is possible to run a stochastic gradient descent (SGD) by exploiting the fact that the cost 7.3 ) is written as an expectation. The randomness in this problem comes from the initial positions $\underline{X}_{0}=\left(\check{X}_{0}^{i, \alpha_{\theta}}\right)_{i}$ and the noise increments $\left(\Delta \breve{W}_{n}\right)_{n=0, \ldots, N_{T}}=\left(\Delta \check{W}_{n}^{i}\right)_{i, n}$. Hence $S=\left(\underline{\underline{X}}_{0},\left(\Delta \underline{\underline{W}}_{n}\right)_{n}\right)$ is going to play the role of a random sample in SGD. Given a realization of $S$ and a choice of parameter $\theta$, we can construct the trajectory $\left(\check{X}_{t_{n}}^{i, \alpha_{\theta}, S}\right)_{i=1, \ldots, N, n=0, \ldots, N_{T}}$ by following $(7.4)$ and we can compute the induced cost:

$$
\mathbf{J}_{S}^{N, \Delta t}(\theta)=\frac{1}{N} \sum_{i=1}^{N}\left[\sum_{n=0}^{N_{T}-1} f\left(\check{X}_{t_{n}}^{i, \alpha_{\theta}, S}, \check{m}_{t_{n}}^{N, \alpha_{\theta}, S}, \alpha_{\theta}\left(t_{n}, \check{X}_{t_{n}}^{i, \alpha_{\theta}, S}\right)\right) \Delta t+g\left(\check{X}_{T}^{i, \alpha_{\theta}, S}, \check{m}_{T}^{N, \alpha_{\theta}, S}\right)\right] .
$$

The SGD procedure in this context is summarized in Algorithm 6. We refer to, e.g., 32 for more details on SGD. The most costly step is the computation of the gradient $\nabla_{\theta} \mathbf{J}_{S}^{N, \Delta t}\left(\theta^{(\mathrm{k})}\right)$ with respect to $\theta$. However, modern programming libraries (such as TensorFlow or PyTorch) allow us to perform this computation automatically using backpropagation, and to adjust the learning rate in an efficient way. The present method is thus very straightforward to implement: contrary to the methods presented in the previous sections, there is no need to derive by hand any PDE, any FBSDE, or any gradient, and one works directly with the definition of the MFC.

Besides this aspect, the main reasons behind the success of this method are the expressive power of neural networks (meaning that complex functions can be well approximated with relatively few parameters) and the fact that there is a priori 
no limitation on the number of iterations K (because the samples $S$ come from Monte-Carlo simulation and not from a finite set of data).

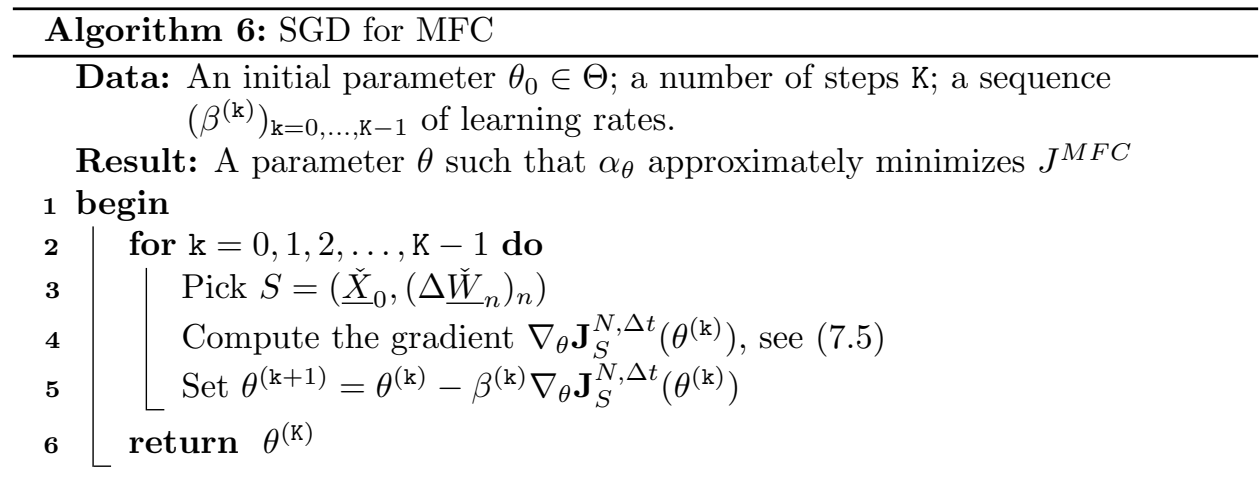

Numerical illustration: LQ MFC. We revisit the linear-quadratic mean field control problem, see $\S 2.6$. We consider an example in dimension $d=10$. For simplicity, we take a setting where the solution is the same in every dimension. More precisely, we take:

$$
A=0, \bar{A}=0, \quad B=Q=\bar{Q}=S=C=Q_{T}=\bar{Q}_{T}=S_{T}=\mathrm{Id}, \quad \sigma=0.1 .
$$

The initial distribution is $\mathcal{N}\left(x_{0}, \sigma_{0}\right)$ with $x_{0}=2$ and $\sigma_{0}=0.2$. In the implementation, we used a feedforward fully connected neural network, as introduced in (7.1), with sigmoid activation function. Figure 19 shows, as a function of $k$, the $L^{2}$ error between the control $\alpha_{\theta^{(\mathrm{k})}}$ learnt by the neural network and the true optimal control $\alpha^{*}$. We see that the error decreases when the number of time steps or the number of particles increase. Although the optimal control is simply a linear function of the state at each time $t$, the neural network does not know this information a priori and nevertheless manages to approximate the optimal solution.
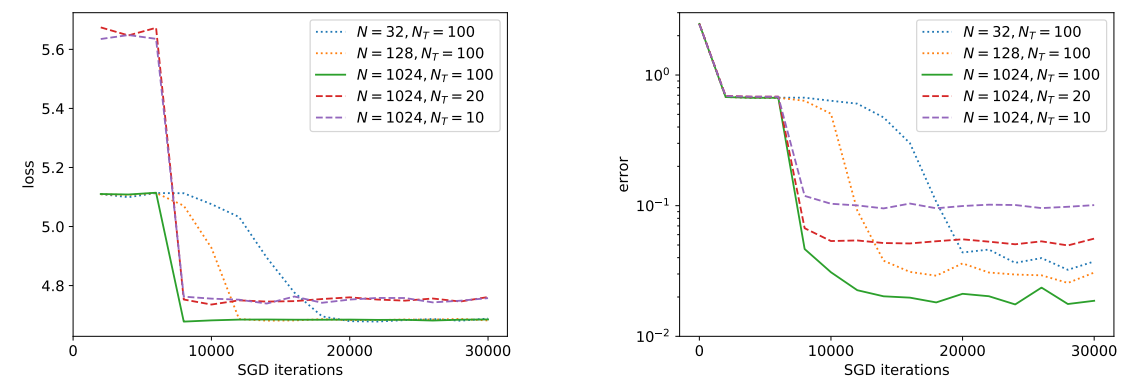

Figure 19. LQ MFC with neural network approximation: total cost (left) and $L^{2}$ error on the optimal control (right) in dimension $d=10$ with $N_{T}$ time steps and $N$ particles as shown in the figures.

Extensions. This technique has already been used for a while for standard stochastic optimal control problems, see e.g., [102, 74, 85. Its flexibility has lead to various applications such as, in the context of MFC, to problems with delay [70] or (biological) neural networks [15. Although it is very well-suited to MFC, this method is not immediately applicable to MFG which do not have a variational structure. However, it is possible to extend this technique to solve 
forward-backward stochastic differential equations (FBSDEs) of McKean-Vlasov type by rewriting the FBSDE as a mean-field optimal control of two forward SDEs with a terminal penalty, see e.g. [55 for more details and numerical results. This line of research extends to the mean-field framework techniques that have been investigated for (non-MKV) FBSDE by E, Han and Jentzen in [66.

7.2. Solving the finite state master equation. All the methods presented so far to solve time-dependent MFC or MFG assume that the initial distribution is fixed. The solution is thus computed to obtain a flow of distribution which starts from this prescribed initial condition. However, in some applications, one does not know for sure the initial distribution. A possible strategy is to repeatedly use one of the methods presented earlier for various initial distributions. However, the computational cost would be prohibitive. It is thus interesting to be able solve the problem for any initial condition at once. This provides a motivation for solving the master equation, which is an equation posed on the space of probability distributions, whose system of characteristics is the forward-backward PDE system introduced in $\S 3.1$. Furthermore, the master equation is also useful to prove convergence of $N$-player games to MFG 42 or to obtain large deviation principles [64.

Master equation for finite state MFG. We focus here on a setting in which the state space is finite. Following the discussion in [52, Section 7.2], let $\mathcal{E}=\left\{e_{1}, \ldots, e_{d}\right\}$ be a finite set, and let $\mathcal{A} \subseteq \mathbb{R}^{k}$ be a Borel set, corresponding respectively to the state space and the action space. We will view $\mathcal{E}$ as a subset of $\mathbb{R}^{d}$ by identifying its elements with the canonical basis, and we will identify $\mathcal{P}(\mathcal{E})$ with the simplex $\left\{m \in \mathbb{R}^{d} \mid \sum_{i=1}^{d} m_{i}=1\right\}$. Let $f: \mathcal{E} \times \mathcal{P}(\mathcal{E}) \times \mathcal{A} \rightarrow \mathbb{R}$ and $g: \mathcal{E} \times \mathcal{P}(\mathcal{E}) \rightarrow \mathbb{R}$ be respectively a running cost and a terminal cost functions. Let $\lambda: \mathcal{E} \times \mathcal{P}(\mathcal{E}) \times \mathcal{A} \rightarrow \mathbb{R}$ be a jump rate function. To alleviate the notation, for every $m \in \mathcal{P}(\mathcal{E})$ we write $m(x)$ instead of $m(\{x\})$. We will write $\mathbb{R}^{\mathcal{E}}$ for the set of functions from $\mathcal{E}$ to $\mathbb{R}$.

We then consider the following MFG equilibrium problem: Find a flow of probability densities $\hat{m}:[0, T] \times \mathcal{E} \rightarrow \mathbb{R}$ and a feedback control $\hat{\alpha}:[0, T] \times \mathcal{E} \rightarrow \mathcal{A}$ satisfying the following two conditions:

(1) $\hat{\alpha}$ minimizes

$$
J_{\hat{m}}^{M F G}: \alpha \mapsto \mathbb{E}\left[\int_{0}^{T} f\left(X_{t}^{\hat{m}, \alpha}, \hat{m}(t, \cdot), \alpha\left(t, X_{t}^{\hat{m}, \alpha}\right)\right) d t+g\left(X_{T}^{\hat{m}, \alpha}, \hat{m}(T, \cdot)\right)\right]
$$

under the constraint that the process $X^{\hat{m}, \alpha}=\left(X_{t}^{\hat{m}, \alpha}\right)_{t \geq 0}$ is a nonhomogeneous $\mathcal{E}$-valued Markov chain with transition probabilities determined by the $Q$-matrix of rates $q^{\hat{m}, \alpha}:[0, T] \times \mathcal{E} \times \mathcal{E} \rightarrow \mathbb{R}$ given by:

$$
q^{\hat{m}, \alpha}\left(t, x, x^{\prime}\right)=\lambda\left(x, x^{\prime}, \hat{m}(t, \cdot), \alpha(t, \cdot)\right), \quad\left(t, x, x^{\prime}\right) \in[0, T] \times \mathcal{E} \times \mathcal{E},
$$

and $X_{0}^{\hat{m}, \alpha}$ has distribution with density $m_{0}$;

(2) For all $t \in[0, T], \hat{m}(t, \cdot)$ is the law of $X_{t}^{\hat{m}, \hat{\alpha}}$.

To formulate an optimality condition, we introduce the Lagrangian $L: \mathcal{E} \times$ $\mathcal{P}(\mathcal{E}) \times \mathbb{R}^{\mathcal{E}} \times \mathcal{A} \rightarrow \mathbb{R}$ defined by:

$$
L(x, m, h, \alpha)=\sum_{x^{\prime} \in \mathcal{E}} \lambda\left(x, x^{\prime}, m, \alpha\right) h\left(x^{\prime}\right)+f(x, m, \alpha),
$$


and the Hamiltonian:

$$
H(x, m, h)=\sup _{\alpha \in \mathcal{A}}-L(x, m, h, \alpha) .
$$

Assuming there is a unique maximizer for every $(x, m, h) \in \mathcal{E} \times \mathcal{P}(\mathcal{E}) \times \mathbb{R}^{\mathcal{E}}$, we denote:

$$
\alpha^{*}(x, m, h)=\underset{\alpha \in \mathcal{A}}{\arg \max }-L(x, m, h, \alpha) .
$$

It will also be useful to introduce the function $q^{*}: \mathcal{E} \times \mathcal{E} \times \mathcal{P}(\mathcal{E}) \times \mathbb{R}^{\mathcal{E}} \rightarrow \mathbb{R}$ defined by

$$
q^{*}\left(x, x^{\prime}, m, h\right)=\lambda\left(x, x^{\prime}, m, \alpha^{*}(x, m, h)\right) .
$$

In the spirit of the forward-backward PDE system, the solution of a finite state MFG can be characterized through a system of ODEs: a forward ODE for the distribution $m:[0, T] \times \mathcal{E} \rightarrow \mathbb{R}$ and a backward ODE for the value function $u:[0, T] \times \mathcal{E} \rightarrow \mathbb{R}$ of an infinitesimal player. To wit, under suitable conditions (see e.g. [52, section 7.2]), there is a unique MFG equilibrium $(\hat{m}, \hat{\alpha})$ and it is given by:

$$
\hat{\alpha}(t, x)=\alpha^{*}(x, m(t, \cdot), u(t, \cdot)),
$$

where $\alpha^{*}$ is defined by $(7.7)$ and $(u, m)$ solves the forward-backward system:

$$
\begin{cases}0=-\partial_{t} u(t, x)+H(x, m(t, \cdot), u(t, \cdot)), & (t, x) \in[0, T) \times \mathcal{E} \\ 0=\partial_{t} m(t, x)-\sum_{x^{\prime} \in \mathcal{E}} m\left(t, x^{\prime}\right) q^{*}\left(x^{\prime}, x, m(t, \cdot), u(t, \cdot)\right), & (t, x) \in(0, T] \times \mathcal{E}, \\ u(T, x)=g(x, m(T, \cdot)), \quad m(0, x)=m_{0}(x), & x \in \mathcal{E}\end{cases}
$$

Since $\mathcal{E}$ is finite, $m(t, \cdot)$ and $u(t, \cdot)$ can be identified with vectors and each equation can be viewed as an ODE. This forward-backward system can be tackled using techniques similar to the ones described in the previous sections for the PDE system arising in the continuous state space case. For instance, one can first discretize time with a semi-implicit scheme and then use fixed point iterations, alternating between the HJB and the FP equation, or use Newton iterations; see $\S 4$.

Due to the coupling between the two equations, the value function $u$ depends implicitly on the distribution $m$. The solution to the master equation allows us to make this dependence explicit. In the present setting, this equation takes the following form (see e.g. [52, section 7.2] for more details):

$$
-\partial_{t} \mathcal{U}(t, x, m)+H(x, m, \mathcal{U}(t, \cdot, m))-\sum_{x^{\prime} \in \mathcal{E}} h^{*}(m, \mathcal{U}(t, \cdot, m))\left(x^{\prime}\right) \frac{\partial \mathcal{U}(t, x, m)}{\partial m\left(x^{\prime}\right)}=0,
$$

for $(t, x, m) \in[0, T] \times \mathcal{E} \times \mathcal{P}(\mathcal{E})$, with the terminal condition $\mathcal{U}(T, x, m)=g(x, m)$, for $(x, m) \in \mathcal{E} \times \mathcal{P}(\mathcal{E})$. Here, $h^{*}:[0, T] \times \mathcal{P}(\mathcal{E}) \times \mathbb{R}^{\mathcal{E}} \times \mathcal{E} \rightarrow \mathbb{R}$ is defined as:

$$
h^{*}(m, u)\left(x^{\prime}\right)=\sum_{x \in \mathcal{E}} \lambda\left(x, x^{\prime}, m, \alpha^{*}(x, m, u)\right) m(x) .
$$

The notation $\frac{\partial \mathcal{U}(t, x, m)}{\partial m\left(x^{\prime}\right)}$ represents the (classical) partial derivative of $\mathbb{R}^{d} \ni m \mapsto$ $\mathcal{U}(t, x, m)$ with respect to the coordinate corresponding to $x^{\prime}$ when $m$ is viewed as a vector of dimension $d$. The link between the master equation and the forwardbackward system is the following. For every initial distribution $m_{0} \in \mathcal{P}(\mathcal{E})$,

$$
\mathcal{U}\left(t, x, m^{m_{0}}(t, \cdot)\right)=u^{m_{0}}(t, x), \quad(t, x) \in[0, T] \times \mathcal{E},
$$


where $\left(u^{m_{0}}, m^{m_{0}}\right)$ is the solution to 7.8 starting with $m(0, \cdot)=m_{0}$. In words, the solution to the forward-backward system plays the role of characteristics for the master equation, and $\mathcal{U}$ explicitly captures the (implicit) dependence of the infinitesimal player's value function $u$ on the population's distribution $m$.

Principle of the Deep Galerkin method. Note that the master equation $(7.9)$ is posed on the space $[0, T] \times \mathcal{E} \times \mathcal{P}(\mathcal{E}) \subset[0, T] \times \mathcal{E} \times \mathbb{R}^{d}$, which is high dimensional as soon as the number of states, $d$, is large. Solving high-dimensional PDEs using for instance finite difference schemes is in general computationally expensive due to the fact that the number of grid points increases exponentially with the dimension. Recently, several methods based on machine learning tools have been developed for this purpose. Here, we propose to use the Deep Galerkin Method (DGM) introduced in 109. to solve the master equation (7.9). The main idea is to first replace the unknown function, namely $\mathcal{U}$ in our case, by a neural network, say $\mathcal{U}_{\theta}$, with parameters $\theta$, and then to optimize over $\theta$ in order to minimize the residual of the PDE (7.9). This optimization is achieved by using SGD in the following way: at each iteration, a point in the domain is picked according to a chosen distribution, then the gradient of the PDE residual with respect to the neural network parameters is computed and a gradient step is performed. The procedure is summarized in Algorithm 7. Here, we fix a neural network architecture and denote by $\Theta$ the set of possible parameters for neural networks with this architecture. For the PDE residual we use the notation: for $\theta \in \Theta$ and $S=(t, x, m) \in[0, T] \times \mathcal{E} \times \mathcal{P}(\mathcal{E})$,

$$
\operatorname{Res}_{S}(\theta)=\left|\partial_{t} \mathcal{U}_{\theta}(S)-H\left(x, m, \mathcal{U}_{\theta}(S)\right)+\sum_{x^{\prime} \in \mathcal{E}} h^{*}\left(m, \mathcal{U}_{\theta}(S)\right)\left(x^{\prime}\right) \frac{\partial \mathcal{U}_{\theta}(S)}{\partial m\left(x^{\prime}\right)}\right|^{2}
$$

In the implementation, the neural network $\mathcal{U}_{\theta}$ is a function of $S$ for which partial derivatives can be computed using automatic differentiation. The computation of the residual can thus be implemented without approximating the derivatives. Then, the gradient $\nabla_{\theta} \operatorname{Res}_{S}(\theta)$ of the residual with respect to $\theta$ can be computed using backpropagation. The DGM amounts to try to minimize the loss function:

$$
L(\theta)=\mathbb{E}_{S \sim \nu}\left[\operatorname{Res}_{S}(\theta)\right] .
$$

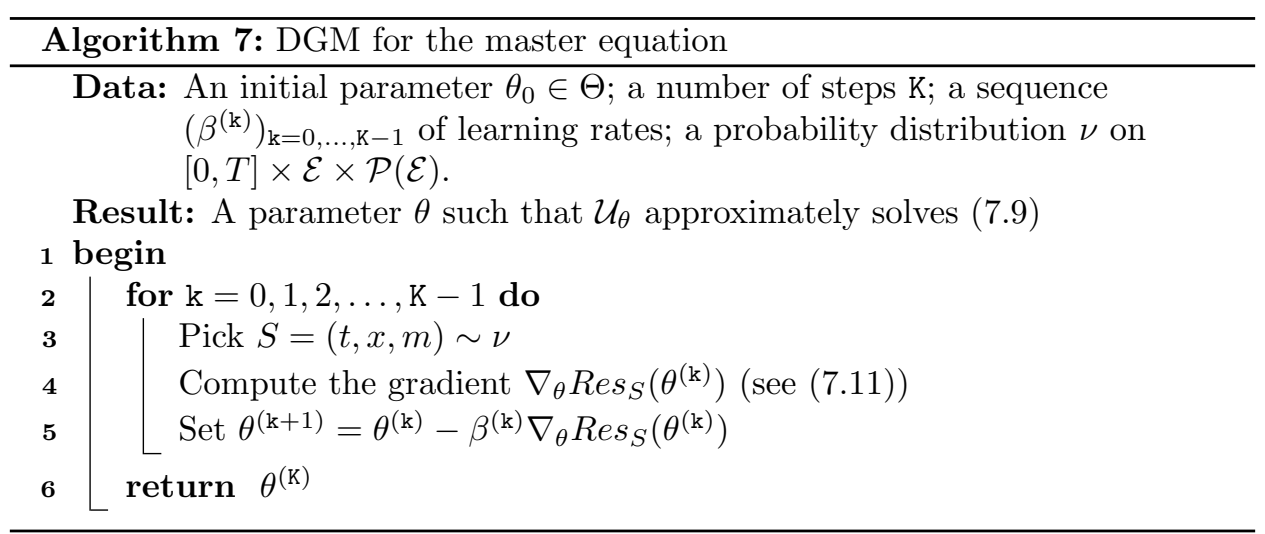

Example 1: A Cybersecurity model. We consider a model introduced in [88 and revisited in [52, Section 7.2.3]. In this model, each player owns a computer which can be either defended (D) or undefended (U), and either infected (I) or susceptible $(\mathrm{S})$ of infection. Hence the set $\mathcal{E}$ has four elements corresponding to the four possible combinations: $\mathcal{E}=\{D I, D S, U I, U S\}$. The action set is $\mathcal{A}=$ 
$\{0,1\}$, where 0 is interpreted as the fact that the player is satisfied with the current level of protection (D or $\mathrm{U}$ ) of its computer, whereas 1 means that she wants to change this level of protection (i.e., she wants to go from $\mathrm{D}$ to $\mathrm{U}$ or vice versa). In the latter case, the update occurs at a (fixed) rate $\rho>0$. At each of the four states, all the computers are indistinguishable. When infected, each computer may recover at rate $q_{r e c}^{D}$ or $q_{r e c}^{U}$ depending on whether it is defended or not. On the other hand, a computer may be infected directly by a hacker, at rate $v_{H} q_{i n f}^{D}$ (resp. $v_{H} q_{\text {inf }}^{U}$ ) if it is defended (resp. undefended), or it may be infected by undefended infected computers, at rate $\beta_{U U} \mu(\{U I\})$ (resp. $\left.\beta_{U D} \mu(\{U I\})\right)$ if it is undefended (resp. defended), or by defended infected computers, at rate $\beta_{D U} \mu(\{D I\})$ (resp. $\left.\beta_{D D} \mu(\{D I\})\right)$ if it is undefended (resp. defended). In short, the matrix of transition rates is given by: for $m \in \mathcal{P}(\mathcal{E}), a \in \mathcal{A}$,

$$
\lambda(\cdot, \cdot, m, a)=\left(\lambda\left(x, x^{\prime}, m, a\right)\right)_{x, x^{\prime} \in \mathcal{E}}=\left(\begin{array}{cccc}
\ldots & P_{D S \rightarrow D I}^{m, a} & \rho a & 0 \\
q_{r e c}^{D} & \ldots & 0 & \rho a \\
\rho a & 0 & \ldots & P_{U S \rightarrow U I}^{m, a} \\
0 & \rho a & q_{r e c}^{U} & \ldots
\end{array}\right)
$$

where

$$
\begin{aligned}
& P_{D S \rightarrow D I}^{m, a}=v_{H} q_{i n f}^{D}+\beta_{D D} m(\{D I\})+\beta_{U D} m(\{U I\}), \\
& P_{U S \rightarrow U I}^{m, a}=v_{H} q_{i n f}^{U}+\beta_{U U} m(\{U I\})+\beta_{D U} m(\{D I\}),
\end{aligned}
$$

and all the instances of ... should be replaced by the negative of the sum of the entries of the row in which ... appears on the diagonal. At each time, the player pays a protection cost $k_{D}>0$ if its computer is defended, and a penalty $k_{I}>0$ if it is infected. There is no terminal cost and, given a mean-field flow $m$ and a control $\alpha$, the instantaneous cost at time $t$ is hence

$$
f\left(X_{t}^{m, \alpha}, m(t, \cdot), \alpha\left(t, X_{t}^{m, \alpha}\right)\right)=-\left[k_{D} \mathbf{1}_{\{D I, D S\}}\left(X_{t}^{m, \alpha}\right)+k_{I} \mathbf{1}_{\{D I, U I\}}\left(X_{t}^{m, \alpha}\right)\right] .
$$

Note that in this example, the cost is independent of the population's distribution and the mean-field interactions are only in the dynamics.

We apply the DGM method described above to the master equation $(7.9)$ in this cyber-security example. We obtain a neural network $\mathcal{U}_{\theta}$ which is an approximation of $\mathcal{U}$. For the sake of comparison, we (separately) solve the forward-backward system (7.8) for various initial distributions $m_{0}$ and obtain solutions $\left(u^{m_{0}}, m^{m_{0}}\right)$. We then compare $\mathcal{U}_{\theta}\left(t, x, m^{m_{0}}(t, \cdot)\right)$ and $u^{m_{0}}(t, x)$ which gives two curves for each $x \in \mathcal{E}$. According to the relation (7.10), we know that these two curves should coincide, and this is verified in our numerical experiments, see Figures 20,22 where we consider three test cases corresponding to three different initial conditions. Here we used the following values for the parameters:

$$
\left\{\begin{array}{l}
\beta_{U U}=0.3, \beta_{U D}=0.4, \beta_{D U}=0.3, \beta_{D D}=0.4, \\
v_{H}=0.2, \lambda=0.5, \\
q_{r e c}^{D}=0.1, q_{r e c}^{U}=0.65, q_{\text {inf }}^{D}=0.4, q_{i n f}^{U}=0.3, \\
k_{D}=0.3, k_{I}=0.5 .
\end{array}\right.
$$

In the implementation, we used a feedforward fully connected neural network, as introduced in (7.1), with sigmoid activation function. For problems in higher dimension, other architectures are sometimes more suitable. 

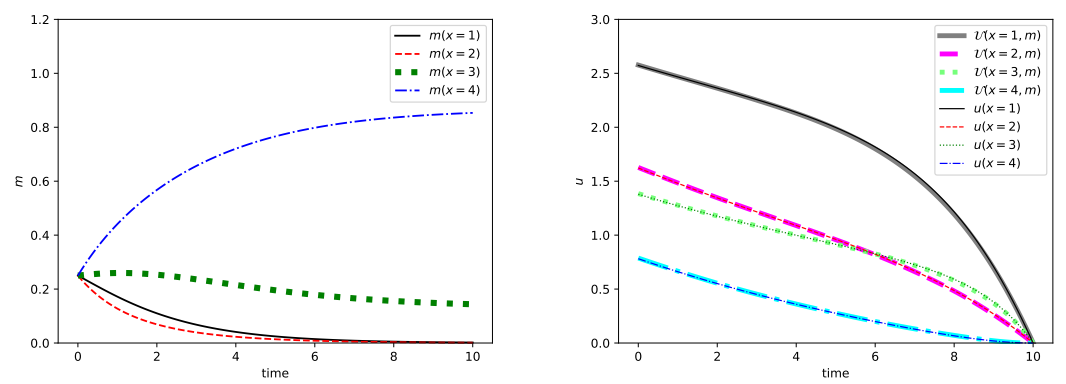

Figure 20. MFG Cyber-security example, test case 1: Evolution of the distribution $m^{m_{0}}$ (left) and the value function $u^{m_{0}}$ and $\mathcal{U}\left(\cdot, \cdot, m^{m_{0}}(\cdot)\right)$ (right) for $m_{0}=(1 / 4,1 / 4,1 / 4,1 / 4)$.
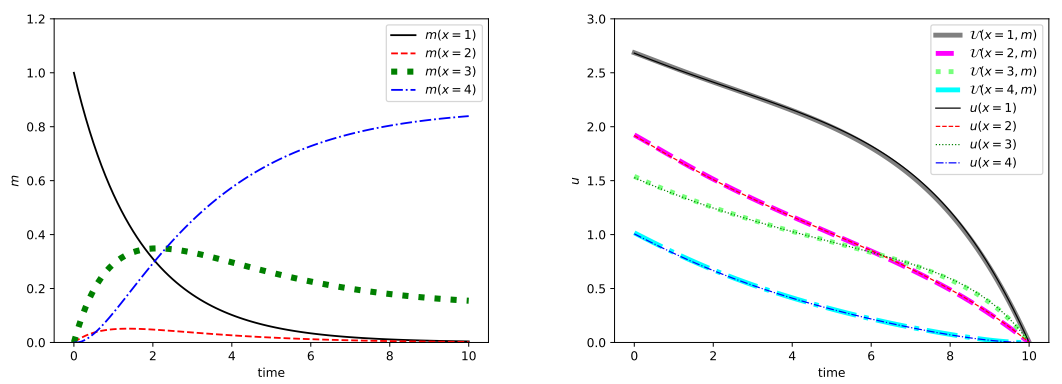

Figure 21. MFG Cyber-security example, test case 2: Evolution of the distribution $m^{m_{0}}$ (left) and the value function $u^{m_{0}}$ and $\mathcal{U}\left(\cdot, \cdot, m^{m_{0}}(\cdot)\right)$ (right) for $m_{0}=(1,0,0,0)$.
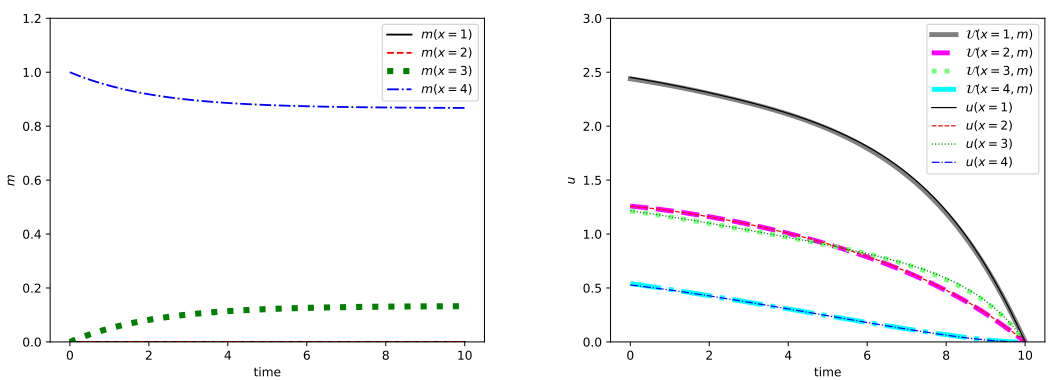

Figure 22. MFG Cyber-security example, test case 3: Evolution of the distribution $m^{m_{0}}$ (left) and the value function $u^{m_{0}}$ and $\mathcal{U}\left(\cdot, \cdot, m^{m_{0}}(\cdot)\right)$ (right) for $m_{0}=(0,0,0,1)$.

Example 2: An Example without uniqueness. In the above example, the solution to the master equation is very smooth. We now turn our attention to an example proposed by Cecchin et al. in [59] for which the master equation has 
multiple solutions but there is only one satisfying an entropy condition. Numerical results show that the neural network manages to approximate this latter solution despite its lack of smoothness.

The state space is $\mathcal{E}=\{-1,1\}$. Since there are only two states, every element of $\mathcal{P}(\mathcal{E})$ can be characterized by its mean $\bar{m}=m(1)-m(-1)$. The transition rate is the control. In other words, at each time $t$, given its state $X_{t}$, an infinitesimal player can choose the rate $\alpha\left(t, X_{t}\right) \in[0,+\infty)$ at which she wants to flip her state. The running and terminal costs are given by:

$$
f(x, m, a)=\frac{a^{2}}{2}, \quad g(x, m)=-x \bar{m} .
$$

The master equation takes the following form: For $(t, x, \bar{m}) \in[0, T] \times\{-1,1\} \times$ $[-1,1]$

$$
\begin{aligned}
-\partial_{t} \mathcal{U}(t, x, \bar{m})+\frac{1}{2}\left[\left(\Delta^{x} \mathcal{U}(t, x, \bar{m})\right)^{-}\right]^{2} \\
\quad-\sum_{y \in\{-1,1\}} \frac{1+y \bar{m}}{2} D^{\bar{m}} \mathcal{U}(t, x, \bar{m}, y)\left(\Delta^{x} \mathcal{U}(t, y, \bar{m})\right)^{-}=0,
\end{aligned}
$$

with the terminal condition $\mathcal{U}(T, x, \bar{m})=-x \bar{m}$, for $(x, \bar{m}) \in\{-1,1\} \times[-1,1]$. Here $\Delta^{x}$ denotes the first difference, defined as:

$$
\Delta^{x} \mathcal{U}(t, x, \bar{m})=\mathcal{U}(t,-x, \bar{m})-\mathcal{U}(t, x, \bar{m}),
$$

and the derivative with respect to $\bar{m}$ is given by:

$$
D^{\bar{m}} \mathcal{U}(t, x, \bar{m}, 1)=2 \partial_{\bar{m}} \mathcal{U}(t, x, \bar{m})=-D^{\bar{m}} \mathcal{U}(t, x, \bar{m},-1),
$$

where $\partial_{\bar{m}}$ denotes a partial derivative with respect to the real variable $\bar{m}$ in the usual sense.

Introducing the new variable:

$$
\mathcal{Z}(t, \bar{m})=\mathcal{U}(T-t,-1, \bar{m})-\mathcal{U}(T-t, 1, \bar{m}), \quad(t, \bar{m}) \in[0, T] \times[-1,1],
$$

we can check that $\mathcal{Z}$ solves:

$$
\begin{cases}\partial_{t} \mathcal{Z}(t, \bar{m})+\partial_{\bar{m}} \mathfrak{g}(\bar{m}, \mathcal{Z}(t, \bar{m}))=0, & (t, \bar{m}) \in[-1,1] \\ \mathcal{Z}(0, \bar{m})=\mathfrak{f}(\bar{m}), & \bar{m} \in[-1,1]\end{cases}
$$

where

$$
\mathfrak{f}(\bar{m})=2 \bar{m}, \quad \mathfrak{g}(\bar{m}, z)=\bar{m} \frac{z|z|}{2}-\frac{z^{2}}{2}, \quad(\bar{m}, z) \in[-1,1] \times \mathbb{R} .
$$

This equation is a scalar conservation law admitting three solutions, among which only one is an entropy solution (see [59. Proposition 3]), which is given by:

$$
\mathcal{Z}(t, \bar{m})=\frac{2 M(t, \bar{m})}{t|M(t, \bar{m})|+1},
$$

where $M(t, 0)=0$ and, if $\bar{m} \neq 0, M(t, \bar{m})$ denotes the unique solution to:

$$
t^{2} M^{3}+t(2-t) M|M|+(1-2 t) M-\bar{m}=0,
$$

with the same sign as $\bar{m}$.

Figure 23 shows the true $\mathcal{Z}$ given by $(7.13)$ and the one obtained by the change of variable (7.12) after the neural network has been trained to approximate $\mathcal{U}$. We see that the true $\mathcal{Z}$ exhibits a discontinuity at $\bar{m}$ after time $t=0.5$, whereas the neural network is continuous because we used the sigmoid function as an activation 
function. However, Figure 24 shows that the $L^{2}$ error decays with the number of SGD iterations, and after a large enough number of iterations, the neural network manages to approximate the discontinuity as shown for the terminal time $T=1$.
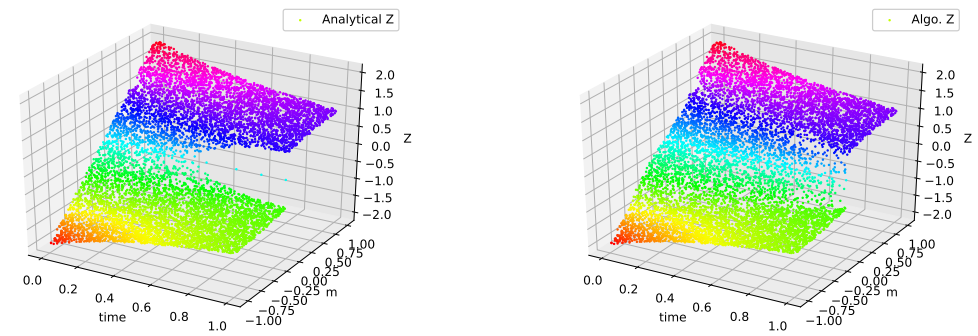

Figure 23. Example with non-uniqueness: $\mathcal{Z}$ from analytical formula (7.13) (left) and from Deep Galerkin Method applied to compute $\mathcal{U}$, from which $\mathcal{Z}$ is deduced by 7.12 (right).
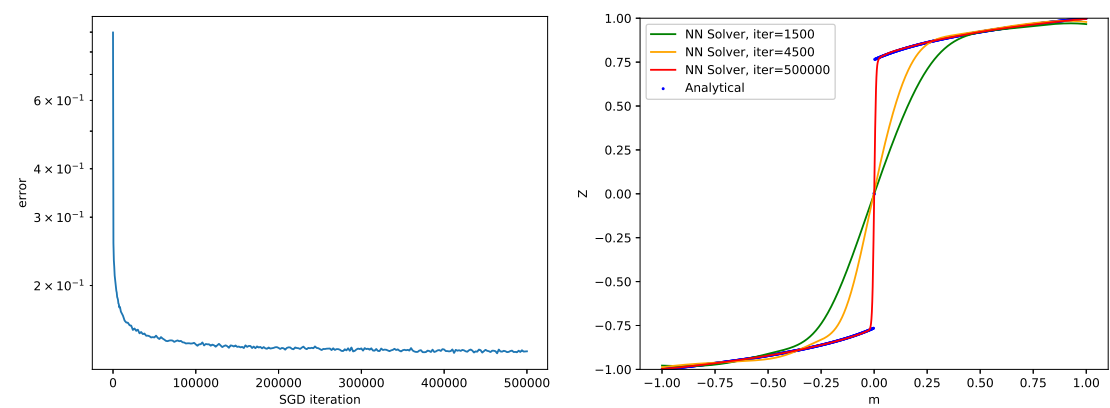

Figure 24. Example with non-uniqueness: error versus number of SGD iterations (left), and $\mathcal{Z}(T, \cdot)$ (right), where the true solution is in blue, and the solution learnt by the neural network is in green, yellow and red for 1500, 4500 and $5 \times 10^{5}$ iterations respectively.

To conclude this section, let us mention that the DGM method can also be used to tackle the system of HJB-KFP PDEs characterizing the MFG or MFC solutions; see 16, 56 for more details. 


\section{A Glance at model-free methods}

All the previous methods rely, in one way or another, on the fact that the cost functions $f$ and $g$ as well as the drift $b$ and the volatility $\sigma$ are known. However, in many applications, coming up with a realistic and accurate model is a daunting task. It is sometimes impossible to guess the form of the dynamics, or the way the costs are incurred. This provides a motivation to study so-called model-free methods. The theory of reinforcement learning (RL) has formalized this framework and numerous algorithms have been developed. Intuitively, an agent evolving in an environment can take actions and observe the consequences of her actions: the state of the environment (or her own state) changes, and a cost is incurred to the agent. The agent does not know how the new state and the cost are computed. The goal for the agent is then to learn an optimal behavior (i.e., which minimizes the sum of future costs) by trial and error. The problem is even more complex if multiple agents try to learn simultaneously and their actions influence each other's costs. We briefly describe some recent progress on the connection between reinforcement learning and mean field problems. We start with MFC, which, as a control problem, can be recast as a RL problem. We then consider MFG, which requires learning a Nash equilibrium.

8.1. Reinforcement learning for mean field control. As discussed in Section 5. MFC problems can be viewed as optimal control problems driven by the evolution of a probability distribution. This corresponds to the viewpoint of a central planner trying to find the optimal control that the agents should use in order to minimize the social cost. To fit more easily in the framework of reinforcement learning, we revisit the setting with finite state and action spaces considered in $\S 7.2$. Instead of the finite horizon setting, we consider here the infinite horizon discounted case. We modify the class of controls and instead of considering functions of $t$ (time) and $x$ (individual state), we consider here functions of $m$ (population distribution) and $x$ (individual state). Intuitively, the reason behind this choice is that in many cases, the optimal control depends on time only through the population's distribution. The MFC counterpart to the MFG is to find a feedback control $\alpha^{*}: \mathcal{P}(\mathcal{E}) \times \mathcal{E} \rightarrow \mathcal{A}$ minimizing:

$$
J^{M F C}: \alpha \mapsto \mathbb{E}\left[\int_{0}^{+\infty} e^{-\beta t} f\left(X_{t}^{\alpha}, m^{\alpha}(t, \cdot), \alpha\left(m^{\alpha}(t, \cdot), X_{t}^{\alpha}\right)\right) d t\right]
$$

under the constraint that the process $X^{\alpha}=\left(X_{t}^{\alpha}\right)_{t \geq 0}$ is a nonhomogeneous $\mathcal{E}$ valued Markov chain with transition probabilities determined by the $Q$-matrix of rates $q^{m^{\alpha}, \alpha}:[0, T] \times \mathcal{E} \times \mathcal{E} \rightarrow \mathbb{R}$ given by 7.6$)$ and $m^{\alpha}$ is the flow of distributions corresponding to $X^{\alpha}$.

Notice that the cost can be rewritten as:

$$
J^{M F C}(\alpha)=\int_{0}^{+\infty} e^{-\beta t} \sum_{x \in \mathcal{E}} f\left(x, m^{\alpha}(t, \cdot), \alpha\left(m^{\alpha}(t, \cdot), x\right)\right) m^{\alpha}(t, x) d t,
$$

subject to the constraint that $m^{\alpha}$ solves an evolution equation analogous to $7.8 \mathrm{~b}$ but controlled by $\alpha$ :

$$
\partial_{t} m^{\alpha}(t, x)=\sum_{x^{\prime} \in \mathcal{E}} m^{\alpha}\left(t, x^{\prime}\right) \lambda\left(x^{\prime}, x, m^{\alpha}(t, \cdot), \alpha\left(m^{\alpha}(t, \cdot), x^{\prime}\right)\right), \quad(t, x) \in[0, T] \times \mathcal{E} .
$$


Discretizing time with a grid $\left\{t_{n}=n \Delta t, n=0,1,2, \ldots\right\}$ with $\Delta t>0$, the cost is approximately equal to:

$$
\begin{aligned}
J^{M F C, \Delta t}(\alpha) & =\sum_{n=0}^{+\infty} e^{-\beta t_{n}} \sum_{x \in \mathcal{E}} f\left(x, m_{n}^{\alpha}, \alpha\left(m_{n}^{\alpha}, x\right)\right) m_{n}^{\alpha}(x) \Delta t \\
& =\sum_{n=0}^{+\infty} \gamma^{n} \tilde{f}\left(m_{n}^{\alpha}, \alpha\left(m_{n}^{\alpha}, \cdot\right)\right),
\end{aligned}
$$

subject to:

$$
m_{n+1}^{\alpha}=\left(m_{n}^{\alpha}\right)^{\top}\left(I+P^{\alpha\left(m_{n}^{\alpha}, \cdot\right), m_{n}^{\alpha}} \Delta t\right)=: \Phi\left(m_{n}^{\alpha}, \alpha\left(m_{n}^{\alpha}, \cdot\right)\right), \quad n=0,1,2, \ldots
$$

with a given initial condition $m_{0}^{\alpha}$, where $\gamma=e^{-\beta \Delta t}, \tilde{f}: \mathcal{P}(\mathcal{E}) \times \mathcal{A}^{\mathcal{E}} \rightarrow \mathbb{R}$ is defined by:

$$
\tilde{f}(m, \tilde{\alpha})=\sum_{x \in \mathcal{E}} f(x, m, \tilde{\alpha}(x)) m(x) \Delta t, \quad(m, \tilde{\alpha}) \in \mathcal{P}(\mathcal{E}) \times \mathcal{A}^{\mathcal{E}},
$$

and

$$
P^{\tilde{\alpha}, m}\left(x^{\prime}, x\right)=\lambda\left(x^{\prime}, x, m, \tilde{\alpha}\left(x^{\prime}\right)\right), \quad\left(x^{\prime}, x, m, \tilde{\alpha}\right) \in \mathcal{E} \times \mathcal{E} \times \mathcal{P}(\mathcal{E}) \times \mathcal{A}^{\mathcal{E}} .
$$

The problem thus fits in the framework of Markov decision processes (MDP for short) by considering the distribution $m^{\alpha}$ as the state (which is consistent with the point of view of the central planner). Instead of considering the dynamics of $X^{\alpha}$ evolving in $\mathcal{E}$, the MDP is for the "lifted" problem driven by the dynamics of $m^{\alpha}$ evolving in $\mathcal{P}(\mathcal{E})$. The value function $V$ associated to this optimal control problem represents the optimal (infinite horizon discounted) cost-to-go starting from a given state. Here, a state is a population distribution and hence the value function is a function of $m \in \mathcal{P}(\mathcal{E})$ :

$$
V(m)=\inf _{\alpha: \mathcal{P}(\mathcal{E}) \times \mathcal{E} \rightarrow \mathcal{A}} \sum_{n=0}^{+\infty} \gamma^{n} \tilde{f}\left(m_{n}^{\alpha}, \alpha\left(m_{n}^{\alpha}, \cdot\right)\right), \quad \text { with } m_{0}^{\alpha}=m .
$$

However, even if we manage to compute $V$, it is not clear how to recover the optimal control if the cost function and the dynamics are not known. From this perspective, a more useful function is the so-called $Q$-function. It is a function of a state and an action, and it represents the optimal cost-to-go given that we start with the prescribed state-action pair (and then we behave optimally). In our context, it takes the following form:

$$
Q(m, \tilde{\alpha})=\tilde{f}(m, \tilde{\alpha})+\gamma \inf _{\alpha: \mathcal{P}(\mathcal{E}) \times \mathcal{E} \rightarrow \mathcal{A}} \sum_{n=0}^{+\infty} \gamma^{n} \tilde{f}\left(m_{n}^{\alpha}, \alpha\left(m_{n}^{\alpha}, \cdot\right)\right), \quad(m, \tilde{\alpha}) \in \mathcal{P}(\mathcal{E}) \times \mathcal{A}^{\mathcal{E}}
$$

where $m_{0}^{\alpha}=\Phi(m, \tilde{\alpha})$ is the distribution obtained by starting from $m$ and moving forward by one time step using action $\tilde{\alpha}$, and for $n \geq 0, m_{n+1}^{\alpha}=\Phi\left(m_{n}^{\alpha}, \alpha\left(m_{n}^{\alpha}, \cdot\right)\right)$. We have

$$
V(m)=\inf _{\tilde{\alpha} \in \mathcal{A}^{\mathcal{E}}} Q(m, \tilde{\alpha}), \quad m \in \mathcal{P}(\mathcal{E}),
$$

and the optimal control is readily recovered from $Q$ as:

$$
\alpha^{*}(m, \cdot)=\underset{\tilde{\alpha} \in \mathcal{A}^{\mathcal{E}}}{\arg \inf } Q(m, \tilde{\alpha}) .
$$


This is one of the main advantages of the state-action value function $Q$ over the (state only) value function $V$. Furthermore, it can be shown that $Q$ satisfies a dynamic programming equation (or Bellman equation):

$$
Q(m, \tilde{\alpha})=\tilde{f}(m, \tilde{\alpha})+\gamma \inf _{\tilde{\alpha}^{\prime} \in \mathcal{A}^{\mathcal{E}}} Q\left(\Phi(m, \tilde{\alpha}), \tilde{\alpha}^{\prime}\right), \quad(m, \tilde{\alpha}) \in \mathcal{P}(\mathcal{E}) \times \mathcal{A}^{\mathcal{E}} .
$$

Thus, a straightforward strategy to compute $Q$ is to iteratively update an approximation by plugging in the right hand side above the previous estimate. This leads to the so-called (synchronous) $Q$-learning updates:

$$
Q^{(\mathrm{k}+1)}(m, \tilde{\alpha})=\tilde{f}(m, \tilde{\alpha})+\gamma \inf _{\tilde{\alpha}^{\prime} \in \mathcal{A}^{\mathcal{E}}} Q^{(\mathrm{k})}\left(\Phi(m, \tilde{\alpha}), \tilde{\alpha}^{\prime}\right), \quad(m, \tilde{\alpha}) \in \mathcal{P}(\mathcal{E}) \times \mathcal{A}^{\mathcal{E}}
$$

Notice that in order to perform this kind of updates, all we need is to have access to the cost $\tilde{f}(m, \tilde{\alpha})$ and to the next state $\Phi(m, \tilde{\alpha})$, which can be provided by some environment whose inner functioning is not revealed to the learning agent. In other words, to perform the $Q$-learning updates, we do not need to know the functions $\tilde{f}$ and $\Phi$. In this sense, the algorithm is model-free.

To implement this method, we need a finite dimensional approximation of the $Q$ function. We do not discuss here function approximation techniques. For simplicity, we replace $\mathcal{P}(\mathcal{E})$ by a discrete version, denoted by $\tilde{\mathcal{P}}_{\mathcal{E}}$, which contains a finite number of points of $\mathcal{P}(\mathcal{E})$. Since $\mathcal{A}^{\mathcal{E}}$ is also a finite set, the $Q$-function can be approximated by its values on $\tilde{\mathcal{P}}_{\mathcal{E}} \times \mathcal{A}^{\mathcal{E}}$, i.e., by a matrix $\tilde{Q} \in \mathbb{R}^{\left|\tilde{\mathcal{P}}_{\mathcal{E}}\right| \times\left|\mathcal{A}^{\mathcal{E}}\right|}$. The update rule become: $(8.4)$

$$
\tilde{Q}^{(\mathrm{k}+1)}(\tilde{m}, \tilde{\alpha})=\tilde{f}(\tilde{m}, \tilde{\alpha})+\gamma \inf _{\tilde{\alpha}^{\prime} \in \mathcal{A}^{\mathcal{E}}} \tilde{Q}^{(\mathrm{k})}\left(\Pi_{\tilde{\mathcal{P}}_{\mathcal{E}}} \Phi(\tilde{m}, \tilde{\alpha}), \tilde{\alpha}^{\prime}\right), \quad(\tilde{m}, \tilde{\alpha}) \in \tilde{\mathcal{P}}(\mathcal{E}) \times \mathcal{A}^{\mathcal{E}} .
$$

where $\Pi_{\tilde{P}_{\mathcal{E}}}: \mathcal{P}_{\mathcal{E}} \rightarrow \tilde{\mathcal{P}}_{\mathcal{E}}$ denotes a projection on $\tilde{\mathcal{P}}_{\mathcal{E}}$ (note that $\Phi(\tilde{m}, \tilde{\alpha})$ is not necessarily an element of $\tilde{P}_{\mathcal{E}}$ ).

Note that in the current setting, in fact it is enough to interact once with the environment and query $(\tilde{f}(\tilde{m}, \tilde{\alpha}), \Phi(\tilde{m}, \tilde{\alpha}))$ for every pair $(\tilde{m}, \tilde{\alpha}) \in \tilde{\mathcal{P}}_{\mathcal{E}} \times \mathcal{A}^{\mathcal{E}}$ in order to know everything we need about the cost and the dynamics to compute the $\tilde{Q}$ function. However, this is no longer true when the dynamics is stochastic, e.g. due to the presence of common noise. Moreover, when the number of states $\left|\tilde{\mathcal{P}}_{\mathcal{E}}\right|$ or the number of actions $\left|\mathcal{A}^{\mathcal{E}}\right|$ is large, updating $\tilde{Q}$ for every pair $(\tilde{m}, \tilde{\alpha}) \in \tilde{\mathcal{P}}_{\mathcal{E}} \times \mathcal{A}^{\mathcal{E}}$ at each iteration is prohibitive. One can rely on asynchronous updates or function approximation to improve efficiency.

For the purpose of illustration, we present the behavior of the basic mean-field $Q$-learning method 8.4 on the cyber-security model presented in $\S 7.2$ but now from the point of view of a central planner controlling a large group of computers. Recall that $\mathcal{E}=\{D I, D S, U I, U S\}$ has four elements. We replace $\mathcal{P}(\mathcal{E})$ by:

$$
\tilde{\mathcal{P}}_{\mathcal{E}}=\left\{\left(m_{D I}, m_{D S}, m_{U I}, m_{U S}\right) \in \mathcal{G}_{N_{m}}^{4} \mid m_{D I}+m_{D S}+m_{U I}+m_{U S}=1\right\},
$$

where $\mathcal{G}_{N_{m}}$ is a uniform grid over [0,1] with $N_{m} \geq 2$ points, which contains 0 and 1. After K steps of $Q$-learning, we obtain an approximation $\tilde{Q}^{(\mathrm{K})}$ of the $Q$ function, from which we can recover an approximation $\tilde{\alpha}^{\mathrm{K}}$ of the optimal control using (8.2), namely: $\tilde{\alpha}^{\mathrm{K}}(m, \cdot)=\arg \max _{\tilde{\alpha} \in \mathcal{A}^{\mathcal{E}}} \tilde{Q}^{(\mathrm{K})}\left(\Pi_{\tilde{P}_{\mathcal{E}}} m, \tilde{\alpha}\right)$. For three different initial conditions, we compare the flow of distributions induced by this control $\tilde{\alpha}^{\mathrm{K}}$ with the optimally controlled flow. In line with (8.1), we also compare the value function $V\left(m^{\alpha^{*}}(t)\right)$ with $\max _{\mathcal{A}^{\mathcal{E}}} \tilde{Q}^{(\mathrm{K})}\left(\Pi_{\tilde{P}_{\mathcal{E}}} m^{\alpha^{*}}(t), \cdot\right)$ along the optimal flow $\left(m^{\alpha^{*}}(t)\right)_{t \in[0, T]}$. Figures 2527 show the results for three initial conditions. We see 
that the learnt $Q$-function approximately matches the $V$ value function. For these simulations, we used $N_{m}=30, \gamma=0.5$, and the following parameters:

$$
\left\{\begin{array}{l}
\beta_{U U}=0.3, \beta_{U D}=0.4, \beta_{D U}=0.3, \beta_{D D}=0.4, \\
v_{H}=0.6, \lambda=0.8 \\
q_{r e c}^{D}=0.5, q_{r e c}^{U}=0.4, q_{i n f}^{D}=0.4, q_{i n f}^{U}=0.3, \\
k_{D}=0.3, k_{I}=0.5 .
\end{array}\right.
$$

As already mentioned, the update rule (8.4) is provided here simply for the purpose of explaining the basic idea of $Q$-learning in a mean-field setup. We refer to $\mathbf{7 2},[\mathbf{5 8}, \mathbf{8 0}, \mathbf{8 1}, 99$ for more details on mean-field MDPs and $Q$-learning for mean field control. Other methods have also been investigated, such as policy gradient, see 110, which can be proved to converge with a linear rate for linearquadratic MFC problems, see [57].
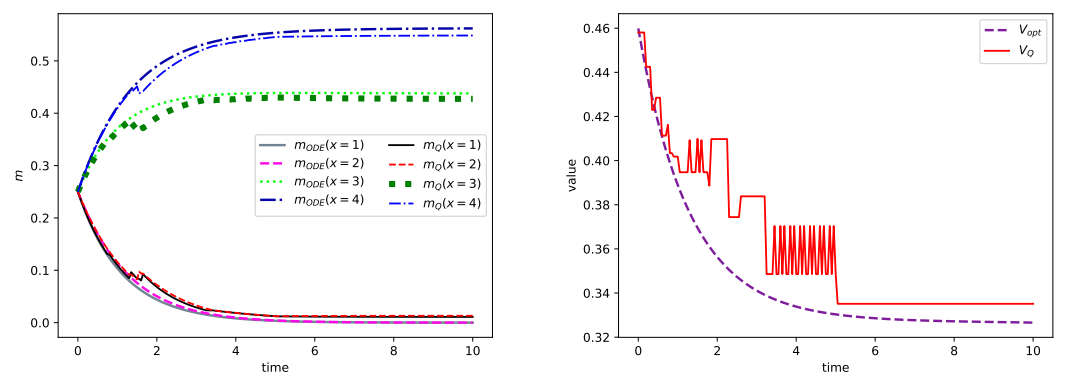

Figure 25. MFC Cyber-security example, test case $1: m_{0}=$ $(1 / 4,1 / 4,1 / 4,1 / 4)$. Left: Evolution of the distribution $m^{m_{0}}$ optimally controlled $\left(m_{O D E}\right)$ or controlled using the approximate $Q$-function $\left(m_{Q}\right)$. Right: $V$ function $\left(V_{o p t}\right)$ and approximate $Q$ function $\left(V_{Q}\right)$ along the optimal flow.

8.2. Reinforcement learning in mean field games. Since MFGs are fixedpoint problems which can not, in general, be written as optimization problems, Nash equilibria can not be immediately recast as MDPs. When the model is not known, it is not clear how to use optimality conditions such as PDE of FBSDE systems. Instead, coming back to the definition (1.1)- 1.2 suggests to iteratively update the distribution and, at each iteration, solve the optimal control problem faced by a typical player using reinforcement learning. This corresponds to the following scenario: an infinitesimal agent can observe a given population distribution and aims at learning her best response by trial and error (i.e., without knowing how her cost or her evolution, which depend on the mean-field term, are computed). This step can be recast as a (standard) MDP parameterized by the mean-field term. The key difference with MFC discussed above, is that the population's distribution does not change while the infinitesimal player learns her optimal control. Then, the mean-field term is updated using this (approximate) best response, and so on. Once again, the update of the distribution can be for instance of Picard type or of fictitious play type. We refer respectively to $[\mathbf{8 2}$ and $[\mathbf{6 8}, \mathbf{1 0 3}$ for more 

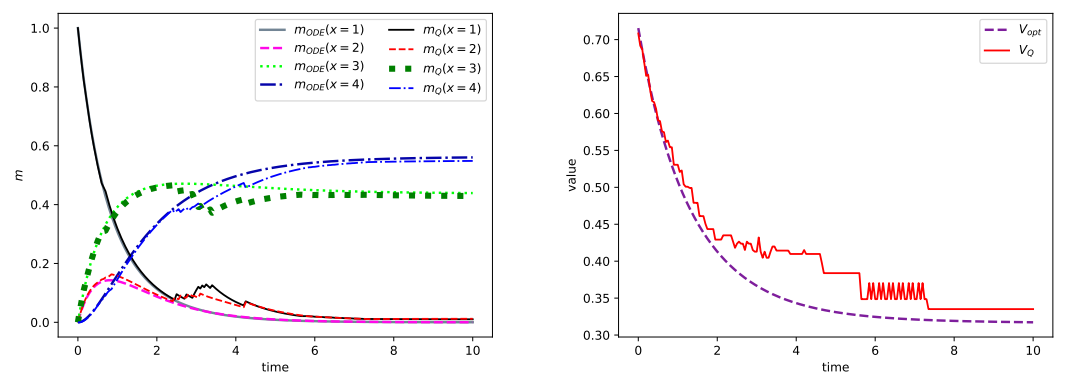

Figure 26. MFC Cyber-security example, test case 2: $m_{0}=$ $(1,0,0,0)$. Left: Evolution of the distribution $m^{m_{0}}$ optimally controlled $\left(m_{O D E}\right)$ or controlled using the approximate $Q$-function $\left(m_{Q}\right)$. Right: $V$ function $\left(V_{o p t}\right)$ and approximate $Q$-function $\left(V_{Q}\right)$ along the optimal flow.
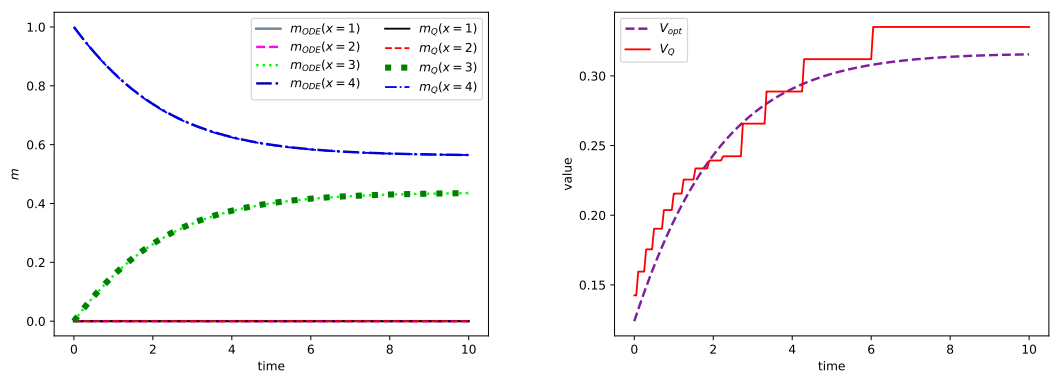

Figure 27. MFC Cyber-security example, test case $3: m_{0}=$ $(0,0,0,1)$. Left: Evolution of the distribution $m^{m_{0}}$ optimally controlled $\left(m_{O D E}\right)$ or controlled using the approximate $Q$-function $\left(m_{Q}\right)$. Right: $V$ function $\left(V_{o p t}\right)$ and approximate $Q$-function $\left(V_{Q}\right)$ along the optimal flow.

details on these methods; see also $\mathbf{1 1 0}$ for a different method based on a twotimescale approach. To conclude this section, let us mention that despite the crucial differences between MFG and MFC, reinforcement learning algorithms for these problems can be unified through the aforementioned two-timescale approach: in fact, solutions to MFG and MFC can be computed by learning simultaneously the value function and the distribution, with different learning rates; see 21 for more details. For the sake of brevity we refrain from developing here RL for MFGs. 


\section{Conclusion and perspectives}

We have presented several aspects of numerical methods for mean field games and mean field control problems. Besides the approaches presented above, several other directions have been investigated, such as (to cite just a few):

- methods for forward-backward system of McKean-Vlasov SDEs: Chaudru de Raynal and Garcia Trillos have proposed in [62 a cubature method for FBSDE of MKV type when the system is decoupled; Chassagneux et al. have proposed in [61, 22 a recursive procedure, while other methods based on neural network approximation are studied in [70, 55, 73. by reformulating the FBSDE system as an optimal control of two forward SDEs where the cost function is a penalty for not matching the terminal condition;

- algorithms for ergodic MFGs: in [37, 35, Cacace et al. proposed an approach relying on a finite difference scheme and a least square formulation, which is then solved using Gauss-Newton iterations;

- gradient-type methods for MFC and variational MFG: see [89, 95, 104] for applications to crowd motion or systemic risk;

- computational methods aiming specifically at MFGs with a non-local dependence on the population distribution, for instance using Fourier expansion techniques [100;

- methods based on machine learning tools besides the ones discussed above: for instance methods based on neural networks for the PDE system of MFG have been investigated in [16, [56, where the value function and the distribution are replaced by two neural networks and the optimization follows the DGM strategy; the link between this idea and Generative Adversarial Networks (GANs) is investigated in [40; on the other hand, 108 proposes a method based on neural network for MFC and variational MFG;

- learning or reinforcement learning methods besides the ones discussed above, such as policy gradient [10], sequential decomposition [98, actorcritic method [71, online mirror descent [83, value iteration [18] or policy iteration [36; see also [11];

- purely probabilistic methods such as quantization or regression based methods for a class of MFC [24], or the approach based Markov chain approximation for MFG studied by Bayraktar et al. in [25.

For the sake of the presentation, the framework used in these notes is often much more restrictive and much simpler than what is covered in the literature. The interested reader is referred to the references cited throughout the text. However, some aspects such as general boundary conditions or general diffusion terms (which could depend on the control and be degenerate) remain challenging and will hopefully be addressed in future works. Besides the development of numerical methods per se, we believe that these tools will unlock many applications for instance in economics, robotics, biology or epidemiology.

Acknowledgements. M. Laurière is very grateful to Yves Achdou, Luis BriceñoArias, René Carmona, François Delarue, Francisco Silva and Xianjin Yang for fruitful discussions, and to the participants of the AMS Short Course for their questions and comments. M. Laurière would like to thank the anonymous referee for their 
insightful comments and suggestions that helped to improve the quality of the manuscript. The research of M. Laurière is supported by NSF grant DMS-1716673 and ARO grant W911NF-17-1-0578.

\section{References}

1. Yves Achdou, Finite difference methods for mean field games, Hamilton-Jacobi equations: approximations, numerical analysis and applications, Lecture Notes in Math., vol. 2074, Springer, Heidelberg, 2013, pp. 1-47. MR 3135339

2. Yves Achdou, Fabio Camilli, and Italo Capuzzo-Dolcetta, Mean field games: numerical methods for the planning problem, SIAM J. Control Optim. 50 (2012), no. 1, 77-109. MR 2888257

3. , Mean field games: convergence of a finite difference method, SIAM J. Numer. Anal. 51 (2013), no. 5, 2585-2612. MR 3097034

4. Yves Achdou and Italo Capuzzo-Dolcetta, Mean field games: numerical methods, SIAM J. Numer. Anal. 48 (2010), no. 3, 1136-1162. MR 2679575

5. Yves Achdou and Ziad Kobeissi, Mean field games of controls: Finite difference approximations, arXiv preprint arXiv:2003.03968 (2020).

6. Yves Achdou and Jean-Michel Lasry, Mean field games for modeling crowd motion, Contributions to Partial Differential Equations and Applications, Springer International Publishing, 2019, pp. 17-42.

7. Yves Achdou and Mathieu Laurière, On the system of partial differential equations arising in mean field type control, Discrete Contin. Dyn. Syst. 35 (2015), no. 9, 3879-3900. MR 3392611

8. Yves Achdou and Mathieu Laurière, Mean Field Type Control with Congestion, Appl. Math. Optim. 73 (2016), no. 3, 393-418. MR 3498932

9. - Mean Field Type Control with Congestion (II): An augmented Lagrangian method, Appl. Math. Optim. 74 (2016), no. 3, 535-578. MR 3575615

10. , Mean field games and applications: Numerical aspects, Mean Field Games, C.I.M.E. Foundation Subseries, vol. 2281, Springer International Publishing, 2020.

11. Yves Achdou, Mathieu Laurière, and Pierre-Louis Lions, Optimal control of conditioned processes with feedback controls, Journal de Mathématiques Pures et Appliquées (2020).

12. Yves Achdou and Victor Perez, Iterative strategies for solving linearized discrete mean field games systems, Netw. Heterog. Media 7 (2012), no. 2, 197-217. MR 2928376

13. Yves Achdou and Alessio Porretta, Convergence of a finite difference scheme to weak solutions of the system of partial differential equations arising in mean field games, SIAM J. Numer. Anal. 54 (2016), no. 1, 161-186. MR 3452251

14. _ Mean field games with congestion, Ann. Inst. H. Poincaré Anal. Non Linéaire 35 (2018), no. 2, 443-480. MR 3765549

15. Nacira Agram, Azzeddine Bakdi, and Bernt Oksendal, Deep learning and stochastic meanfield control for a neural network model, Available at SSRN 3639022 (2020).

16. Ali Al-Aradi, Adolfo Correia, Danilo Naiff, Gabriel Jardim, and Yuri Saporito, Solving nonlinear and high-dimensional partial differential equations via deep learning, arXiv preprint arXiv:1811.08782 (2018).

17. Noha Almulla, Rita Ferreira, and Diogo Gomes, Two numerical approaches to stationary mean-field games, Dyn. Games Appl. 7 (2017), no. 4, 657-682. MR 3698446

18. Berkay Anahtarci, Can Deha Kariksiz, and Naci Saldi, Value iteration algorithm for meanfield games, arXiv preprint arXiv:1909.01758 (2019).

19. Daniel Andersson and Boualem Djehiche, A maximum principle for SDEs of mean-field type, Appl. Math. Optim. 63 (2011), no. 3, 341-356. MR 2784835

20. Roman Andreev, Preconditioning the augmented Lagrangian method for instationary mean field games with diffusion, SIAM J. Sci. Comput. 39 (2017), no. 6, A2763-A2783. MR 3731033

21. Andrea Angiuli, Jean-Pierre Fouque, and Mathieu Laurière, Unified reinforcement Qlearning for mean field game and control problems, arXiv preprint arXiv:2006.13912 (2020).

22. Andrea Angiuli, Christy V. Graves, Houzhi Li, Jean-François Chassagneux, François Delarue, and René Carmona, Cemracs 2017: numerical probabilistic approach to mfg, ESAIM: ProcS 65 (2019), 84-113 
23. Alexander Aurell and Boualem Djehiche, Mean-field type modeling of nonlocal crowd aversion in pedestrian crowd dynamics, SIAM J. Control Optim. 56 (2018), no. 1, 434-455. MR 3763083

24. Alessandro Balata, Côme Huré, Mathieu Laurière, Huyên Pham, and Isaque Pimentel, A class of finite-dimensional numerically solvable McKean-Vlasov control problems, ESAIM: Proceedings and Surveys 65 (2019), 114-144.

25. Erhan Bayraktar, Amarjit Budhiraja, and Asaf Cohen, A numerical scheme for a mean field game in some queueing systems based on Markov chain approximation method, SIAM J. Control Optim. 56 (2018), no. 6, 4017-4044. MR 3873029

26. Erhan Bayraktar, Andrea Cosso, and Huyên Pham, Randomized dynamic programming principle and Feynman-Kac representation for optimal control of McKean-Vlasov dynamics, Trans. Amer. Math. Soc. 370 (2018), no. 3, 2115-2160. MR 3739204

27. Jean-David Benamou and Yann Brenier, A computational fluid mechanics solution to the Monge-Kantorovich mass transfer problem, Numer. Math. 84 (2000), no. 3, 375-393. MR 1738163

28. Jean-David Benamou and Guillaume Carlier, Augmented Lagrangian methods for transport optimization, mean field games and degenerate elliptic equations, J. Optim. Theory Appl. 167 (2015), no. 1, 1-26. MR 3395203

29. Jean-David Benamou, Guillaume Carlier, and Filippo Santambrogio, Variational mean field games, Active particles. Vol. 1. Advances in theory, models, and applications, Model. Simul. Sci. Eng. Technol., Birkhäuser/Springer, Cham, 2017, pp. 141-171. MR 3644590

30. Alain Bensoussan, Jens Frehse, and Sheung Chi Phillip Yam, Mean field games and mean field type control theory, Springer Briefs in Mathematics, Springer, New York, 2013.

31. On the interpretation of the Master Equation, Stochastic Process. Appl. 127 (2017), no. 7, 2093-2137. MR 3652408

32. Léon Bottou, Frank E. Curtis, and Jorge Nocedal, Optimization methods for large-scale machine learning, SIAM Rev. 60 (2018), no. 2, 223-311. MR 3797719

33. Luis M. Briceño Arias, Dante Kalise, Ziad Kobeissi, Mathieu Laurière, Álvaro Mateos González, and Francisco J. Silva, On the implementation of a primal-dual algorithm for second order time-dependent mean field games with local couplings, ESAIM: ProcS 65 (2019), 330-348.

34. Luis M. Briceño Arias, Dante Kalise, and Francisco J. Silva, Proximal methods for stationary mean field games with local couplings, SIAM J. Control Optim. 56 (2018), no. 2, 801-836. MR 3772008

35. Simone Cacace, Fabio Camilli, Annalisa Cesaroni, and Claudio Marchi, An ergodic problem for mean field games: qualitative properties and numerical simulations, Minimax Theory Appl. 3 (2018), no. 2, 211-226. MR 3882530

36. Simone Cacace, Fabio Camilli, and Alessandro Goffi, A policy iteration method for mean field games, arXiv preprint arXiv:2007.04818 (2020).

37. Simone Cacace, Fabio Camilli, and Claudio Marchi, A numerical method for mean field games on networks, ESAIM Math. Model. Numer. Anal. 51 (2017), no. 1, 63-88. MR 3601001

38. Peter E. Caines, Minyi Huang, and Roland P. Malhamé, Handbook of dynamic game theory, t. basar and g. zaccour (eds.), Springer, Cham, 2017.

39. Fabio Camilli, Serikbolsyn Duisembay, and Qing Tang, Approximation of an optimal control problem for the time-fractional fokker-planck equation, arXiv preprint arXiv:2006.03518 (2020).

40. Haoyang Cao, Xin Guo, and Mathieu Laurière, Connecting gans and mfgs, arXiv preprint arXiv:2002.04112 (2020)

41. Pierre Cardaliaguet, Notes on mean field games, 2013.

42. Pierre Cardaliaguet, François Delarue, Jean-Michel Lasry, and Pierre-Louis Lions, The master equation and the convergence problem in mean field games, Annals of Mathematics Studies, vol. 201, Princeton University Press, Princeton, NJ, 2019. MR 3967062

43. Pierre Cardaliaguet and P. Jameson Graber, Mean field games systems of first order, ESAIM Control Optim. Calc. Var. 21 (2015), no. 3, 690-722. MR 3358627

44. Pierre Cardaliaguet, P. Jameson Graber, Alessio Porretta, and Daniela Tonon, Second order mean field games with degenerate diffusion and local coupling, NoDEA Nonlinear Differential Equations Appl. 22 (2015), no. 5, 1287-1317. MR 3399179 
45. Pierre Cardaliaguet and Saeed Hadikhanloo, Learning in mean field games: the fictitious play, ESAIM Control Optim. Calc. Var. 23 (2017), no. 2, 569-591. MR 3608094

46. Pierre Cardaliaguet, Alpár R. Mészáros, and Filippo Santambrogio, First order mean field games with density constraints: pressure equals price, SIAM J. Control Optim. 54 (2016), no. 5, 2672-2709. MR 3556062

47. Elisabetta Carlini and Francisco J. Silva, A fully discrete semi-Lagrangian scheme for a first order mean field game problem, SIAM J. Numer. Anal. 52 (2014), no. 1, 45-67. MR 3148086

48. _ A semi-Lagrangian scheme for a degenerate second order mean field game system, Discrete Contin. Dyn. Syst. 35 (2015), no. 9, 4269-4292. MR 3392626

49. - On the discretization of some nonlinear Fokker-Planck-Kolmogorov equations and applications, SIAM J. Numer. Anal. 56 (2018), no. 4, 2148-2177. MR 3828859

50. René Carmona, Applications of mean field games to economic theory, Proc. AMS Short Course, arXiv preprint arXiv:2012.05237 (2020).

51. René Carmona and François Delarue, Forward-backward stochastic differential equations and controlled McKean-Vlasov dynamics, Ann. Probab. 43 (2015), no. 5, 2647-2700. MR 3395471

52. _ Probabilistic theory of mean field games with applications. I, Probability Theory and Stochastic Modelling, vol. 83, Springer, Cham, 2018, Mean field FBSDEs, control, and games. MR 3752669

53. _ Probabilistic theory of mean field games with applications. II, Probability Theory and Stochastic Modelling, vol. 84, Springer, Cham, 2018, Mean field games with common noise and master equations. MR 3753660

54. René Carmona, Christy V. Graves, and Zongjun Tan, Price of anarchy for mean field games, CEMRACS 2017-numerical methods for stochastic models: control, uncertainty quantification, mean-field, ESAIM Proc. Surveys, vol. 65, EDP Sci., Les Ulis, 2019, pp. 349-383. MR 3968548

55. René Carmona and Mathieu Laurière, Convergence analysis of machine learning algorithms for the numerical solution of mean field control and games: Ii-the finite horizon case, arXiv preprint arXiv:1908.01613 (2019).

56. - Convergence analysis of machine learning algorithms for the numerical solution of mean field control and games: I-the ergodic case, To appear in SIAM Journal on Numerical Analysis (2021).

57. René Carmona, Mathieu Laurière, and Zongjun Tan, Linear-quadratic mean-field reinforcement learning: convergence of policy gradient methods, arXiv preprint arXiv:1910.04295 (2019).

58. __ Model-free mean-field reinforcement learning: mean-field MDP and mean-field Qlearning, arXiv preprint arXiv:1910.12802 (2019).

59. Alekos Cecchin, Paolo Dai Pra, Markus Fischer, and Guglielmo Pelino, On the convergence problem in mean field games: a two state model without uniqueness, SIAM J. Control Optim. 57 (2019), no. 4, 2443-2466. MR 3981375

60. Antonin Chambolle and Thomas Pock, A first-order primal-dual algorithm for convex problems with applications to imaging, J. Math. Imaging Vision 40 (2011), no. 1, 120-145. MR 2782122

61. Jean-François Chassagneux, Dan Crisan, and François Delarue, Numerical method for FBSDEs of McKean-Vlasov type, Ann. Appl. Probab. 29 (2019), no. 3, 1640-1684. MR 3914553

62. Paul-Eric Chaudru de Raynal and Camilo A. Garcia Trillos, A cubature based algorithm to solve decoupled McKean-Vlasov forward-backward stochastic differential equations, Stochastic Process. Appl. 125 (2015), no. 6, 2206-2255. MR 3322862

63. François Delarue, Master equation and mean field games, Proc. AMS Short Course (2020).

64. François Delarue, Daniel Lacker, and Kavita Ramanan, From the master equation to mean field game limit theory: large deviations and concentration of measure, Ann. Probab. 48 (2020), no. 1, 211-263. MR 4079435

65. Mao Fabrice Djete, Dylan Possamaï, and Xiaolu Tan, McKean-Vlasov optimal control: the dynamic programming principle, arXiv preprint arXiv:1907.08860 (2019).

66. Weinan E, Jiequn Han, and Arnulf Jentzen, Deep learning-based numerical methods for high-dimensional parabolic partial differential equations and backward stochastic differential equations, Commun. Math. Stat. 5 (2017), no. 4, 349-380. MR 3736669 
67. Jonathan Eckstein and Dimitri P. Bertsekas, On the Douglas-Rachford splitting method and the proximal point algorithm for maximal monotone operators, Math. Programming 55 (1992), no. 3, Ser. A, 293-318. MR 1168183

68. Romuald Elie, Julien Pérolat, Mathieu Laurière, Matthieu Geist, and Olivier Pietquin, On the convergence of model free learning in mean field games, AAAI, 2020.

69. Michel Fortin and Roland Glowinski, Augmented Lagrangian methods, Studies in Mathematics and its Applications, vol. 15, North-Holland Publishing Co., Amsterdam, 1983, Applications to the numerical solution of boundary value problems, Translated from the French by B. Hunt and D. C. Spicer. MR 724072

70. Jean-Pierre Fouque and Zhaoyu Zhang, Deep learning methods for mean field control problems with delay, Frontiers in Applied Mathematics and Statistics 6(11) (2020).

71. Zuyue Fu, Zhuoran Yang, Yongxin Chen, and Zhaoran Wang, Actor-critic provably finds nash equilibria of linear-quadratic mean-field games, arXiv preprint arXiv:1910.07498 (2019).

72. Nicolas Gast, Bruno Gaujal, and Jean-Yves Le Boudec, Mean field for Markov decision processes: from discrete to continuous optimization, IEEE Trans. Automat. Control $\mathbf{5 7}$ (2012), no. 9, 2266-2280. MR 2968782

73. Maximilien Germain, Joseph Mikael, and Xavier Warin, Numerical resolution of mckeanvlasov fbsdes using neural networks, arXiv preprint arXiv:1909.12678 (2019).

74. Emmanuel Gobet and Rémi Munos, Sensitivity analysis using Itô-Malliavin calculus and martingales, and application to stochastic optimal control, SIAM J. Control Optim. 43 (2005), no. 5, 1676-1713. MR 2137498

75. Diogo A. Gomes, Edgard A. Pimentel, and Vardan Voskanyan, Regularity theory for meanfield game systems, SpringerBriefs in Mathematics, Springer, [Cham], 2016. MR 3559742

76. Diogo A. Gomes and João Saúde, Mean field games models-a brief survey, Dyn. Games Appl. 4 (2014), no. 2, 110-154. MR 3195844

77. Diogo A. Gomes and João Saúde, Numerical methods for finite-state mean-field games satisfying a monotonicity condition, Applied Mathematics \& Optimization (2018).

78. Gomes, Diogo A. and Yang, Xianjin, The hessian riemannian flow and newton's method for effective hamiltonians and mather measures, ESAIM: M2AN 54 (2020), no. 6, 1883-1915.

79. Christy Graves and Roland P. Malhamé, Mean field games : A paradigm for individual-mass interactions, Proc. AMS Short Course (2020).

80. Haotian Gu, Xin Guo, Xiaoli Wei, and Renyuan Xu, Dynamic programming principles for learning MFCs, arXiv preprint arXiv:1911.07314 (2019).

81. Mean-field controls with Q-learning for cooperative MARL: Convergence and complexity analysis, arXiv preprint arXiv:2002.04131 (2020).

82. Xin Guo, Anran Hu, Renyuan Xu, and Junzi Zhang, Learning mean-field games, in proc. of NeurIPS, 2019.

83. Saeed Hadikhanloo, Learning in mean field games, Ph.D. thesis, University Paris-Dauphine, 2018.

84. Saeed Hadikhanloo and Francisco J. Silva, Finite mean field games: fictitious play and convergence to a first order continuous mean field game, J. Math. Pures Appl. (9) 132 (2019), 369-397. MR 4030259

85. Jiequn Han and Weinan E, Deep learning approximation for stochastic control problems, Deep Reinforcement Learning Workshop, NIPS, arXiv preprint arXiv:1611.07422 (2016).

86. Minyi Huang, Peter E. Caines, and Roland P. Malhamé, Large-population cost-coupled LQG problems with nonuniform agents: individual-mass behavior and decentralized $\epsilon$-Nash equilibria, IEEE Trans. Automat. Control 52 (2007), no. 9, 1560-1571. MR 2352434

87. Minyi Huang, Roland P. Malhamé, and Peter E. Caines, Large population stochastic dynamic games: closed-loop McKean-Vlasov systems and the Nash certainty equivalence principle, Commun. Inf. Syst. 6 (2006), no. 3, 221-251. MR 2346927

88. Vassili N. Kolokoltsov and Alain Bensoussan, Mean-field-game model for botnet defense in cyber-security, Appl. Math. Optim. 74 (2016), no. 3, 669-692. MR 3575619

89. Aimé Lachapelle and Marie-Therese Wolfram, On a mean field game approach modeling congestion and aversion in pedestrian crowds, Transportation research part B: methodological 45 (2011), no. 10, 1572-1589.

90. Daniel Lacker, Notes for the AMS short course on mean field games: The convergence problem, Proc. AMS Short Course (2020). 
91. Jean-Michel Lasry and Pierre-Louis Lions, Jeux à champ moyen. I. Le cas stationnaire, C. R. Math. Acad. Sci. Paris 343 (2006), no. 9, 619-625. MR 2269875

92. _ Jeux à champ moyen. II. Horizon fini et contrôle optimal, C. R. Math. Acad. Sci. Paris 343 (2006), no. 10, 679-684. MR 2271747

93. __ Mean field games, Jpn. J. Math. 2 (2007), no. 1, 229-260. MR 2295621

94. Mathieu Laurière and Olivier Pironneau, Dynamic programming for mean-field type control, C. R. Math. Acad. Sci. Paris 352 (2014), no. 9, 707-713. MR 3258261

95. _ Dynamic programming for mean-field type control, J. Optim. Theory Appl. 169 (2016), no. 3, 902-924. MR 3501391

96. Pierre-Louis Lions, Cours du Collège de France, https://www.college-de-france.fr/site/ en-pierre-louis-lions/_course.htm 2007-2011.

97. Alpár Richárd Mészáros and Francisco J. Silva, A variational approach to second order mean field games with density constraints: the stationary case, J. Math. Pures Appl. (9) 104 (2015), no. 6, 1135-1159. MR 3420414

98. Rajesh K Mishra, Deepanshu Vasal, and Sriram Vishwanath, Model-free reinforcement learning for non-stationary mean field games, 2020 59th IEEE Conference on Decision and Control (CDC), IEEE, 2020, pp. 1032-1037.

99. Médéric Motte and Huyên Pham, Mean-field markov decision processes with common noise and open-loop controls, arXiv preprint arXiv:1912.07883 (2019).

100. Levon Nurbekyan et al., Fourier approximation methods for first-order nonlocal mean-field games, Portugaliae Mathematica 75 (2019), no. 3, 367-396.

101. Marcel Nutz and Yuchong Zhang, Conditional optimal stopping: a time-inconsistent optimization, Ann. Appl. Probab. 30 (2020), no. 4, 1669-1692. MR 4133380

102. Thomas Parisini and R Zoppoli, Neural approximations for multistage optimal control of nonlinear stochastic systems, IEEE Transactions on Automatic Control 41 (1996), no. 6, 889-895.

103. Sarah Perrin, Julien Pérolat, Mathieu Laurière, Matthieu Geist, Romuald Elie, and Olivier Pietquin, Fictitious play for mean field games: Continuous time analysis and applications, in proc. of NeurIPS, 2020.

104. Laurent Pfeiffer, Numerical methods for mean-field type optimal control problems, Pure Appl. Funct. Anal. 1 (2016), no. 4, 629-655. MR 3619691

105. Huyên Pham and Xiaoli Wei, Dynamic programming for optimal control of stochastic McKean-Vlasov dynamics, SIAM J. Control Optim. 55 (2017), no. 2, 1069-1101. MR 3631380

106. Kavita Ramanan, Deviations and fluctuations for mean field games, Proc. AMS Short Course (2020).

107. R. Tyrrell Rockafellar, Convex analysis, Princeton Landmarks in Mathematics, Princeton University Press, Princeton, NJ, 1997, Reprint of the 1970 original, Princeton Paperbacks.

108. Lars Ruthotto, Stanley J Osher, Wuchen Li, Levon Nurbekyan, and Samy Wu Fung, A machine learning framework for solving high-dimensional mean field game and mean field control problems, Proceedings of the National Academy of Sciences 117 (2020), no. 17, 91839193.

109. Justin Sirignano and Konstantinos Spiliopoulos, DGM: a deep learning algorithm for solving partial differential equations, J. Comput. Phys. 375 (2018), 1339-1364. MR 3874585

110. Jayakumar Subramanian and Aditya Mahajan, Reinforcement learning in stationary meanfield games, in proc. of AAMAS, 2019.

111. Hamidou Tembine, Raul Tempone, and Pedro Vilanova, Mean-field learning: a survey, arXiv preprint arXiv:1210.4657 (2012).

Princeton University, Operations Research and Financial Engineering (ORFE) Department, Sherrerd Hall, Princeton, New Jersey, U.S.A.

E-mail address: lauriere@princeton.edu 